\title{
Partial Nephrectomy for the Treatment of Renal Cell Carcinoma and the Risk of End Stage Renal Disease
}

\author{
by
}

Stanley A. Yap, MD

A thesis submitted in conformity with the requirements for the degree of Masters of Science in Clinical Epidemiology Institute of Health Policy, Management, and Evaluation University of Toronto

2013

(C) Copyright by Stanley Alexander Yap (2013) 


\title{
Partial Nephrectomy for the Treatment of Renal Cell Carcinoma and the Risk of End Stage Renal Disease
}

\author{
Stanley A. Yap, MD \\ Masters of Science in Clinical Epidemiology \\ Institute of Health Policy, Management, and Evaluation \\ University of Toronto
}

2013

\begin{abstract}
The surgical management of renal masses involves either radical nephrectomy $(\mathrm{RN})$ or partial nephrectomy (PN). PN has been associated with better long-term renal function, and has also been linked to improved cardiac morbidity and overall survival. However, the relationship between treatment choice and definitive outcomes of chronic kidney disease (CKD) are lacking. Our aim was to examine whether PN is associated with a lower risk of end-stage renal disease (ESRD) requiring renal replacement therapy.

We performed a population-based, retrospective cohort study with data from linked administrative databases in the province of Ontario, Canada. We included individuals with pathologically confirmed renal cell carcinoma (RCC) diagnosed between 1995 and 2010. Cox proportional hazards (PH) models, propensity score models, and competing risks models were used to assess the impact of treatment choice.

PN compared to $\mathrm{RN}$ reduces the risk of ESRD requiring renal replacement therapy in a modern cohort of patients (2003-2010). PN is associated with a lower risk of new-onset CKD, lower risk of cardiac morbidity, and improved overall survival. We provide further evidence for the benefit of $\mathrm{PN}$ compared to $\mathrm{RN}$, particularly related to definitive outcomes of renal failure.
\end{abstract}




\section{ACKNOWLEDGEMENTS}

This would not have been possible without the support of many individuals, and I would like to express my sincere gratitude to all those involved in the project. I would like to especially acknowledge a few individuals who have been the most meaningful to me during this process.

Shabbir Alibhai for his mentorship and guidance. He has demonstrated to me the virtues of organization, patience, thoroughness, and discipline. He has instilled in me many foundations of scientific integrity and method. His enthusiasm will always motivate me, and I have found in him both a mentor and a friend.

Antonio Finelli for his support and mentorship in both my academic and clinical work. This project would not have been possible without him. One of the reasons I pursued my fellowship in Toronto was for his guidance as a clinician scientist. His influence will be the starting point of my own career, and I will always consider him both a mentor and a friend.

David Urbach for his insightful feedback and expertise in health services research. His experience was integral in the design and implementation of this study.

George Tomlinson for his expertise in statistics and his willingness to provide assistance. He possesses a rare talent to make the most daunting concepts seem accessible.

Joseph Kim and Stephen Pautler for their time and expertise as reviewers. Their efforts are appreciated and their feedback will undoubtedly improve the quality of this work.

To my family, my colleagues, and my bel for your friendship, humor, and constant encouragement. 


\section{TABLE OF CONTENTS}

Acknowledgements...........................................................

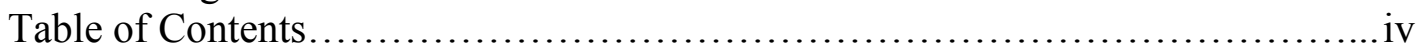

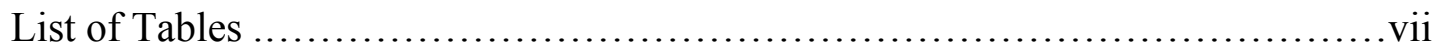

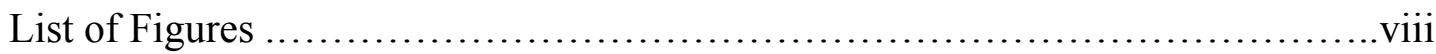

Chapter 1 Introduction and Study Objectives................................ 1

1.1 Rationale....................................................... 1

1.2 Study Objectives................................................. 2

1.3 Study Hypothesis................................................. 2

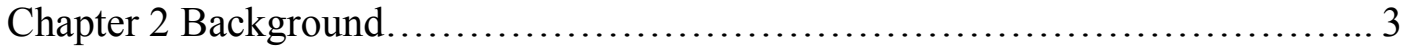

2.1 Epidemiology of Kidney Cancer................................... 3

2.2 Rising incidence over time....................................... 4

2.3 Current management of RCC.....................................4

2.3.1 Surgical approaches................................... 5

2.3.2 Non-surgical approaches................................... 7

2.4 Locally Advanced and Metastatic RCC............................... 9

2.5 Treatment of small renal masses..................................... 9

2.5.1 The Current Standard - Partial Nephrectomy.................9

2.5.2 Equivalent Oncologic Outcomes........................ 10

2.5.3 Equivalent Complication Rates............................11

2.5.4 Benefits of Nephron-sparing............................ 12

2.6 Underutilization of Partial Nephrectomy............................. 14

2.6.1 Overall rates........................................... 14

2.6.2 High-risk Subgroups.................................. 15

2.6.3 Reasons for underuse................................... 16

2.7 Limitations in the Literature......................................... 17

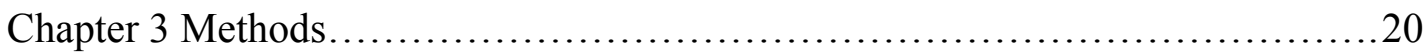

3.1 Study design....................................................... 20

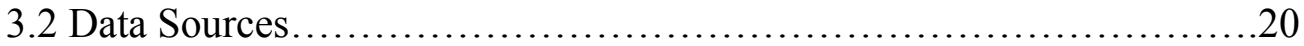

3.2.1 Ontario Cancer Registry..................................20

3.2.2 Institute for Clinical Evaluative Sciences....................20

3.2.3 Canadian Institute for Health Information (CIHI) .............21

3.2.4 Ontario Health Insurance Plan Database..................... 22

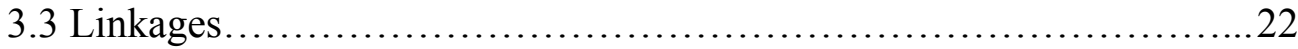

3.4 Ethics and Confidentiality......................................... 23

3.5 Dataflow.................................................... 23

3.6 Population (Inclusion/Exclusions) .............................. 24

3.7 Covariables.................................................. 26

3.7.1 Demographic data.................................... 26

3.7.2 Renal cell carcinoma.....................................26

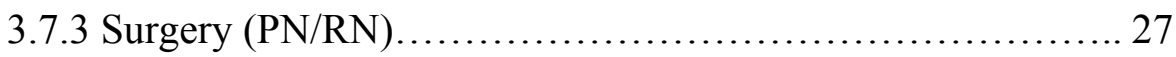

3.7.4 Chronic dialysis...................................... 28

3.7 .5 Acute dialysis......................................... 29

3.7.6 Renal transplantation...................................29

3.7.7 Renal Replacement...................................... 30 
3.7.8 Comorbidity Adjustment.................................... 30

Charlson-Deyo comorbidity score..........................31

Johns Hopkins Adjusted Clinical Groups System.............32

Diabetes mellitus......................................... 33

Hypertension........................................... 33

Myocardial infarction................................... 34

Chronic kidney disease................................... 35

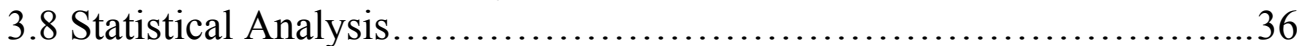

3.8.1 Power calculation.............................................

3.8.2 Data checks............................................... 37

3.8.3 Descriptive analyses.................................... 37

3.8.4 Cox proportional hazards model............................... 37

3.8.5 Propensity score model..................................... 38

3.8.6 Competing risks analysis............................... 40

3.8.7 Subset Analysis - Modern cohort...........................41

Chapter 4 Results............................................................... 43

4.1 Descriptive statistics......................................... 43

4.1.1 Data Checks.............................................. 45

4.1.2 Descriptive statistics - Outcomes.......................... 49

4.1.3 Development of propensity scores.........................51

4.2 Primary outcome - ESRD requiring RRT ..........................5 54

4.3 Primary outcome - ESRD requiring RRT (Modern cohort, 2003-2010).59

4.4 Secondary outcome - Myocardial infarction......................... 63

4.5 Secondary outcome - CKD ...................................... 68

4.6 Secondary outcome - Acute dialysis............................... 72

4.7 Secondary outcome - Overall survival............................ 75

4.8 Secondary outcome - Cancer-specific survival........................78

4.9 Summary of Findings............................................. 82

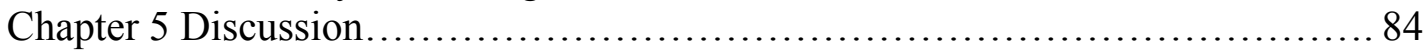

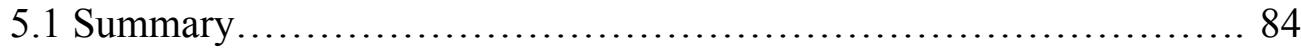

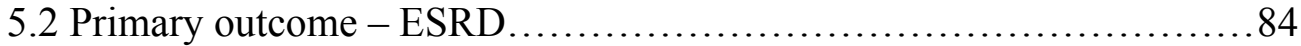

5.3 Secondary outcomes............................................ 87

5.3.1 Chronic kidney disease (CKD) ......................... 87

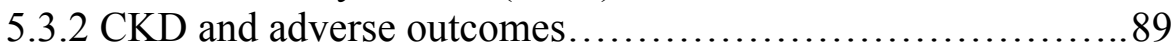

5.3.3 Medical versus Surgical CKD ...............................91

5.3.4 Myocardial infarction...................................99

5.3.5 Acute dialysis.......................................... 94

5.3.6 Overall survival...........................................96

5.3.7 Controversy regarding the EORTC clinical trial............... 96

5.4 Findings (General trends) ....................................... 98

5.4.1 Utilization of PN....................................... 98

5.4.2 Predictors of adverse outcome.................................. 99

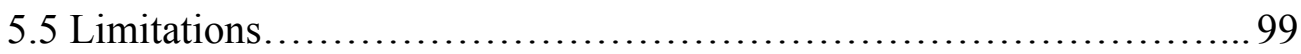

5.5.1 Pathologic and staging data................................ 99

5.5.2 Plasma Creatinine levels........................................ 101

5.5.3 Accuracy of capture of confounders........................ 102 
5.5.4 Ability to control for confounding...................... 104

5.5.5 Analysis of modern cohort............................. 105

5.5.6 Further analysis........................................ 106

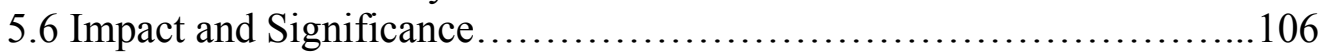

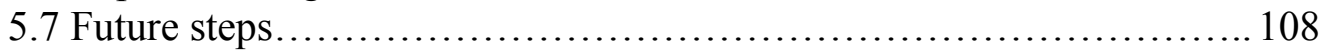

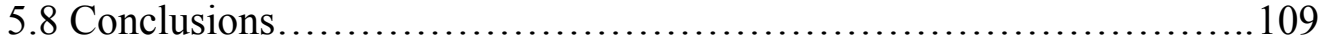

Glossary of Abbreviations................................................. 114

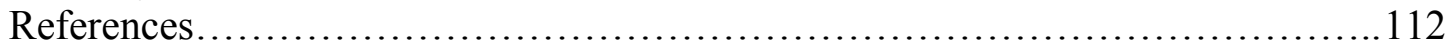

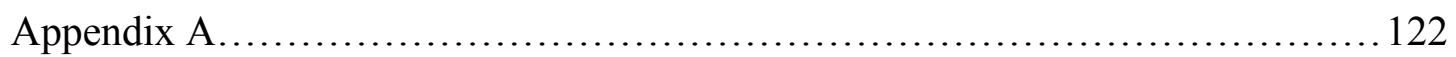

Appendix B........................................................ 129 


\section{List of Tables}

Table 3.1: ICD-9/10 diagnostic codes for renal cell carcinoma

Table 3.2: CCP/CCI procedural codes for radical and partial nephrectomy

Table 3.3: OHIP billing codes for acute and chronic dialysis

Table 3.4: CCP/CCI procedural codes for renal transplantation

Table 3.5: ICD9/10 diagnostic codes for various comorbidity

Table 4.1: Baseline characteristics

Table 4.2: Overall rates of primary and secondary outcomes

Table 4.3: Univariable Cox proportional hazards model (ESRD)

Table 4.4: Univariable and multivariable Cox proportional hazards model with individual comorbidities (ESRD)

Table 4.5: Univariable and multivariable Cox proportional hazards model with individual comorbidities in the modern cohort - 2003-2010 (ESRD)

Table 4.6: Analysis of the impact of PN on outcome of ESRD analyzing sequentially by year to define the modern cohort

Table 4.7: Univariable and multivariable Cox proportional hazards model (MI)

Table 4.8: Univariable and multivariable Cox proportional hazards model (new-onset CKD)

Table 4.9: Univariable and multivariable Cox proportional hazards model (acute dialysis)

Table 4.10: Univariable and multivariable Cox proportional hazards model (overall survival)

Table 4.11: Univariable and multivariable Cox proportional hazards model (cancer-specific survival)

Table 4.12: Summary of findings for the impact of PN compared to RN across various outcomes and analysis methods

Appendix A, Table 1: Distribution of demographic and clinical variables within each propensity score quintile

Appendix A Table 2: Multivariable Cox proportional hazards model of risk of requiring renal replacement therapy with Charlson comorbidity score

Appendix A Table 3: Multivariable Cox proportional hazards model of risk of requiring renal replacement therapy with weighted ADG score

Appendix A Table 4: Cox proportional hazards model adjusting for propensity score as a continuous and categorical variable for the outcome of a) ESRD; b) ESRD (modern cohort); c) new onset CKD; d) myocardial infarction; e) acute dialysis; f) overall survival; g) cancer-specific survival Appendix A Table 5: Baseline characteristics of the modern cohort (2003-2010) 


\section{List of Figures}

Figure 3.1: Dataflow

Figure 3.2: Flowchart for identification of final cohort

Figure 4.1: a) Numbers of cases of PN and RN performed in the province of Ontario (1995-2010)

b) Percentage of cases performed as either RN or PN by year in the province of Ontario.

Figure 4.2: Rates of ESRD by post-operative quarter

Figure 4.3: Rates of individually captured comorbidity across years

Figure 4.4: Rates of RRT across years stratified by surgical treatment

Figure 4.5: Rates of ESRD (\%) stratified by Charlson comorbidity score

Figure 4.6: Rates of ESRD (\%) stratified by weighted ADG score (quintiles)

Figure 4.7: Rates of ESRD (\%) stratified by pre-operative comorbidity

Figure 4.8: Rates of ESRD (\%) by stratified by age group

Figure 4.9: Histogram demonstrating range and distribution of propensity scores

Figure 4.10: Kaplan Meier curves stratified by surgical treatment for outcome of ESRD requiring RRT

Figure 4.11: Competing risks analysis: cumulative probability curves stratified by surgical treatment for the outcome of ESRD requiring RRT.

Figure 4.12: Kaplan Meier curves stratified by surgical treatment for outcome of ESRD requiring RRT (modern cohort, 2003-2010).

Figure 4.13: Competing risks analysis: cumulative probability curves stratified by surgical treatment for the outcome of ESRD requiring RRT in the modern cohort (2003-2010).

Figure 4.14: Kaplan Meier curves stratified by surgical treatment for outcome of MI.

Figure 4.15: Competing risks analysis: cumulative probability curves stratified by surgical treatment for the outcome of hospitalization for MI.

Figure 4.16: Kaplan Meier curves stratified by surgical treatment for outcome of developing newonset CKD.

Figure 4.17: Competing risks analysis: cumulative probability curves stratified by surgical treatment for the outcome of the development of new-onset CKD.

Figure 4.18: Competing risks analysis: cumulative probability curves stratified by surgical treatment for the outcome of acute dialysis

Figure 4.19: Kaplan Meier curves stratified by surgical treatment for outcome of overall survival. Figure 4.20: Kaplan Meier curves stratified by surgical treatment for outcome of cancer-specific survival. 


\section{CHAPTER 1 INTRODUCTION AND STUDY OBJECTIVES}

\subsection{Rationale}

The incidence of small renal masses (SRMs) is rising, and such lesions can be surgically managed with either radical nephrectomy (RN) or partial nephrectomy (PN). These masses are most commonly malignant and equivalent 10-year oncologic outcomes have been demonstrated between the two surgical approaches. ${ }^{1}$ However, PN is associated with better long-term renal function compared to $\mathrm{RN}^{2}$, and has also been linked to improved outcomes regarding cardiac morbidity and overall survival ${ }^{3,4}$. Hence, PN is now recommended as the standard treatment for all $\mathrm{T} 1 \mathrm{a}(\leq 4 \mathrm{~cm})$ lesions. Intuitively, this will be particularly relevant in 'vulnerable' patient populations (i.e. at risk of chronic kidney disease (CKD)), including those with hypertension or diabetes mellitus.

Although studies have demonstrated higher rates of CKD in patients undergoing $\mathrm{RN}$, we lack studies assessing the relationship between treatment choice ( $\mathrm{RN}$ versus $\mathrm{PN}$ ) and end stage renal disease (ESRD) necessitating dialysis or renal transplantation. Renal replacement therapy for ESRD is associated with significant patient morbidity and enormous health resource utilization. Of further concern is a persistent underutilization of PN with an actual decrease in uptake recently demonstrated in Ontario coinciding with the dissemination of laparoscopic RN. ${ }^{5}$ Such surgical choices likely affect long-term outcomes, and may disproportionately impact patients at the highest risk for worsening CKD. The lack of definitive data demonstrating less risk of dialysis or renal transplantation with PN compared to RN may explain the poor uptake in Ontario and other jurisdictions. Our aim is to examine whether PN is associated with a lower risk of requiring dialysis or renal transplantation, particularly amongst vulnerable individuals. 


\subsection{Study Objectives}

$\underline{\text { Primary }}$

To study whether partial nephrectomy is associated with a lower risk of ESRD requiring renal replacement therapy compared to radical nephrectomy.

$\underline{\text { Secondary }}$

a) To study whether partial nephrectomy is associated with a lower risk of CKD, myocardial infarction, acute dialysis or overall mortality compared to radical nephrectomy.

b) To study the impact of preoperative morbidity (diabetes mellitus, HTN) on the subsequent development of ESRD in patients undergoing partial nephrectomy compared to radical nephrectomy.

\subsection{Hypothesis}

Patients undergoing partial nephrectomy for the treatment of renal cell carcinoma will have lower rates of ESRD than those undergoing radical nephrectomy. 


\section{CHAPTER 2 BACKGROUND}

\subsection{Epidemiology of Kidney Cancer}

Although kidney cancer is the $3^{\text {rd }}$ most common genitourinary malignancy, it is the most lethal. There were approximately 271,000 new cases diagnosed worldwide in 2008 and there is a rising incidence, particularly for early stage disease. ${ }^{6,7}$ The vast majority of kidney cancers will be a histologic subtype of renal cell carcinoma (RCC). The most common of these subtypes is clear cell carcinoma (70-80\%), while the remaining subtypes consist of papillary - type 1 and 2(10-15\%) and chromophobe (3$5 \%$ ). RCC is diagnosed twice as often in men as it is in women and the mean age of diagnosis is 70 years old. ${ }^{8}$ Incidence rates vary among ethnic groups, with African American representing one of the highest risk groups and Asians representing one of the lowest risk groups. ${ }^{8}$ Major modifiable risk factors demonstrated in population studies include cigarette smoking ${ }^{9}$ and obesity ${ }^{10}$. Increased incidence of RCC has also been associated with hypertension and acquired renal cystic disease ${ }^{11}$. Finally, RCC is associated with a strong genetic component. The genetic foundation of this disease rests largely on alterations of the Von Hippel-Lindau (VHL) gene located on chromosome 3p. Loss of the VHL gene results in a dysregulation of hypoxia-inducing factor (HIF) pathways, resulting in increased angiogenesis and cell growth through ubiquitin-mediated degradation. Familial syndromes account for approximately $2 \%$ of all $\mathrm{RCC}^{12}$, but genetic mutations are intimately involved in sporadic RCC as well. Familial predisposition has also been reported in sporadic cases not associated with distinct syndromes. The majority of incident cases of RCC represent localized disease, though 5-20\% of patients will have metastatic disease at the time of presentation. ${ }^{13}$ 


\subsection{Rising incidence over time}

The worldwide incidence of renal tumours has risen steadily over the past 3-4 decades, reaching a plateau only in recent years. This rise in incidence is at least partially attributable to the increased utilization of abdominal imaging and a subsequent increase in the diagnosis of incidental renal masses, but other factors are also likely at play. An analysis of the SEER database between the years of 1975 and 1995 has demonstrated a yearly increase in incidence rates of RCC between $2.3-4.3 \%$ depending on gender and race. ${ }^{14} \mathrm{~A}$ more contemporary assessment of the SEER database has demonstrated an increase in incidence rates in the United States from 7.1 to 10.8 per 100,000 between 1983 and 2002. The majority of this increase is attributable to detection of SRMs $(<4 \mathrm{~cm})$, and localized stage I disease now represents $>50 \%$ of newly diagnosed renal tumours. ${ }^{15}$ The landscape of RCC is increasingly dominated by localized and early-stage disease. ${ }^{16}$

\subsection{Current management of RCC}

There is a spectrum of treatment modalities and approaches currently available for the management of RCC. The manner in which these tumours are managed has evolved over the years as we have gained knowledge in the biology and natural history of these tumours, and also as we have developed and refined technologies. Treatments are increasingly tailored to the individual patient, and the risks and benefits of each approach must be weighed against the morbidity and preferences of the patient. We briefly review the major treatment options available for localized kidney cancer including surgical 
removal, ablative therapies, and active surveillance. We also review the treatment options for those with locally advanced or metastatic RCC.

\subsubsection{Surgical approaches}

For patients with localized kidney cancer, surgery still represents the treatment of choice and the current gold standard. Surgical approaches include either radical nephrectomy ( $\mathrm{RN}$ ) or partial nephrectomy (PN). The choice of which surgery to perform depends on many factors, and it is not always a simple decision. Factors to consider include tumour characteristics (size, location), patient comorbidities (age, renal function, diabetes, cardiac disease), and individual patient preferences. Both RN and PN can be successfully performed through either open or laparoscopic approaches.

\section{Radical Nephrectomy}

RN was historically the gold standard for the treatment of all renal masses. This surgery involves removal of the entire kidney along with the surrounding tissue within Gerota's Fascia to obtain a wide surgical margin. ${ }^{17}$ In its original description, the ipsilateral adrenal gland was routinely excised, though current data does not necessarily support this practice unless there is specific concern for adrenal involvement. ${ }^{18-21} \mathrm{RN}$ can be performed through either an open or laparoscopic approach. Outcomes for laparoscopic $\mathrm{RN}$ are equivalent to those of the open approach in appropriately selected patients, and this technique represents the current standard approach for most individuals. $^{22-24}$ 
Among all the treatment options, the greatest experience and the best long-term outcome data are with RN. Five-year recurrence free survival ranges from $91-92 \%$ and 5-year cancer-specific survival from 92-98\% for those undergoing $\mathrm{RN}$, and outcomes are not significantly different between laparoscopic and open approaches. ${ }^{22,25}$ The most commonly reported complications include bleeding, pleural damage, and infection. Rates of such complications remain acceptably low, with significant bleeding reported in anywhere from $1-9 \%$ and pleural damage in $4-9 \%$ of cases. ${ }^{26,27}$

Although RN was historically the standard treatment for all renal masses, it is no longer considered necessary for all tumours. $\mathrm{RN}$ is currently indicated for tumours $\geq$ stage T1b $(>4 \mathrm{~cm})$ and for T1a $(\leq 4 \mathrm{~cm})$ tumours when nephron-sparing approaches are not technically feasible.

\section{Partial Nephrectomy}

$\mathrm{PN}$ is the current gold standard for the surgical treatment of T1a $(\leq 4 \mathrm{~cm})$ renal tumours. PN involves removing only the tumour and a small amount of surrounding renal parenchyma, thus leaving the majority of the kidney intact. Historically, PN was used primarily in situations where nephron-sparing was an absolute indication such as patients with solitary kidneys, multiple bilateral tumours, or significant renal insufficiency. ${ }^{28}$ Indications for PN have since broadened as the safety and the benefits of this approach have become increasingly apparent. ${ }^{29-31}$ The benefit of this approach is that it allows for the adequate removal of the kidney tumour, while preserving as much renal parenchyma as possible. In appropriately selected patients, $\mathrm{PN}$ is associated with equivalent 10 -year disease-free survival and local recurrence rates when compared to RN. ${ }^{1,32,33}$ 
Additionally, PN is associated with better long-term renal function and may play a critical role in improving subsequent outcomes related to cardiac morbidity and overall survival. ${ }^{2-4}$ Although well-established in the treatment of T1a renal tumours, the role of PN for the treatment of larger masses is still being evaluated. Early series have shown promising results for both long-term oncologic control and for the benefits in renal function, with equivalent 5-year CSS of $>90 \%$ when utilizing either PN or RN for T1b tumours. ${ }^{34-36}$ Increased utilization of laparoscopic and robotic approaches in performing PN, which has been demonstrated as a technically feasible approach, have also been observed. $^{34}$

\subsubsection{Non-surgical approaches}

\section{Ablative Therapy}

In recent years, we have seen further developments and increased utilization of ablative therapies, most notably radiofrequency ablation and cryotherapy. These techniques involve destruction of tumour tissue either by burning (radiofrequency) or freezing (cryotherapy). The benefit of these approaches is their minimally invasive nature. Both methods of ablation are delivered through either a percutaneous or laparoscopic approach and are associated with a shorter hospitalization, faster convalescence and reduced morbidity. ${ }^{37}$ Additionally, ablative therapies offer the benefit of a nephron-sparing approach and have minimal impact of renal function. ${ }^{38}$

These techniques are typically reserved for highly selected patients with masses that are both smaller $(\leq 4 \mathrm{~cm}$ in diameter) and ideally located in order to achieve maximum efficacy. Intermediate follow-up on these patients with 5-year recurrence-free survival 
rates have recently been reported at approximately $90 \%{ }^{39,40}$ The vast majority of complications are minor and have been reported at rates of $8-15 \% .{ }^{41,42}$ The most commonly reported complication is pain or paresthesia at the probe site. Although surgical treatments offer slightly superior outcomes regarding recurrence rates, ablative therapies still provide a reasonable alternative in the treatment of SRMs, especially for the elderly, those with significant comorbidity, or those otherwise at increased risk for undergoing a surgical procedure.

\section{Active surveillance}

Historically, surveillance strategies were reserved for patients with significant comorbidities who would not tolerate traditional surgical treatments and were ultimately managed with "watchful waiting". We now have a better understanding of the overall rates of benign disease and the indolent nature of many of these tumours, and active surveillance is considered a reasonable option in many patients presenting with T1a tumours. $^{43-45}$

This approach offers many benefits, including the potential to delay or avoid unnecessary treatment in a subset of patients without losing the ability to adequately treat these tumours if they demonstrate more aggressive behaviour. These strategies are often used in conjunction with percutaneous renal biopsy to assist in the classification of these

tumours prior to embarking on a surveillance protocol. ${ }^{46,47}$ Patients on active surveillance require close monitoring, typically with repeat imaging at 3-6 month intervals. A shift to active treatment can occur at any point and is often triggered by interval growth, dimensions approaching thresholds for certain therapies (ablative therapy, partial 
nephrectomy), or patient anxiety. Although PN remains the standard of care for T1a tumours, active surveillance is an option in the appropriately selected patient and will likely be further embraced in coming years as wider experience is gained and management protocols become refined.

\subsection{Locally Advanced and Metastatic RCC}

The management of metastatic RCC has evolved significantly. Several classes of systemic therapies have entered the market in the past decade and become an integral component of the treatment algorithm for managing advanced and metastatic RCC. Some of these successful therapies include Sunitinib (a tyrosine kinase inhibitor) ${ }^{48,49}$, Sorafenib (a Raf kinase inhibitor) $^{50}$, Bevacizumab (an antibody to VEGF) ${ }^{51}$, and Temsirolimus (an mTOR inhibitor $)^{52}$. These options now offer new hopes in an area that has historically been lacking in successful treatments. These therapies have been used alone and also in the setting of cytoreductive nephrectomy, with demonstrated improvements in survival in the range of 3 to 10 months. ${ }^{53,54}$ Continued rapid development in the management of this phase of the disease is expected.

\subsection{Treatment of Small Renal Masses}

\subsubsection{The Current Standard - Partial Nephrectomy}

In the past, PN was reserved for cases in which nephron-sparing techniques were an absolute indication (i.e. solitary kidneys, bilateral tumours, chronic kidney disease). Technique and experience in PN improved through the 1990's and many series have reported the technical success of this procedure. Subsequently, indications for partial 
nephrectomy expanded to patients with normal contralateral kidneys and without absolute indications related to concerns of chronic renal insufficiency. Furthermore, as we gained longer follow-up on these patients, multiple studies demonstrated equivalent oncologic outcomes with similar complication rates for the treatment of T1a tumours. ${ }^{31,55}$ Concurrently, PN was demonstrated as superior to RN with regards to its nephronsparing benefits and better preservation of renal function. Further associations were then described between the preservation of renal function and long-term outcomes of cardiac morbidity and overall survival. ${ }^{2-4}$

Current guidelines from the American, European, and Canadian Urologic Associations all recommend PN as the standard treatment for T1a renal tumours. ${ }^{43,56,57}$ In 2008, approximately $45 \%$ of patients surgically managed for T1a tumours in the United States underwent PN rather than RN ${ }^{58}$ Results from Canadian population studies have demonstrated parallel patterns of dissemination with a slow but steady uptake of PN

over the past decade. ${ }^{5} \mathrm{PN}$ is slowly being embraced for the treatment of renal tumours but this has required convincing evidence in the following domains: equivalent oncologic outcomes, equivalent complication rates, and the benefit of nephron-sparing (renal function, cardiac disease, overall survival). We review the data supporting these domains below.

\subsubsection{Equivalent Oncologic Outcomes}

The initial critical step in establishing PN as the standard of care for treating T1a tumours was to establish its oncologic equivalence to the previous standard of RN. One of the major concerns with PN is the possibility of positive margins. Rates of positive 
surgical margins after PN are acceptably low, ranging from $0-7 \% .{ }^{59}$ More importantly, margin positivity has not been consistently demonstrated to impact outcomes of metastatic progression or mortality. ${ }^{60-62}$ Five-year local recurrence rates for $\mathrm{PN}$ are reported at $1-10 \%{ }^{63-65}$ Rates of progression to distant metastasis are nearly equivalent between RN (3.7\%) and PN (2.7\%). ${ }^{66,67}$ Cancer-specific survival (5-year) is $>90 \%$ regardless of which surgical approach is utilized for the treatment of SRMs. ${ }^{32,67-69}$

\subsubsection{Equivalent Complication Rates}

The majority of studies comparing RN to PN report overall low rates of morbidity and mortality that are equivalent between the procedures. It appears that rates of adverse outcomes are more attributable to patient age and other comorbidity, rather than the surgical procedure. ${ }^{70-72}$ Other studies have demonstrated similar overall complications rates, though the nature of the complications may be what differentiates the procedures. Greater rates of renal and urinary-specific complications (i.e. bleeding, urinary fistula) have been associated with PN, and higher rates of cardiac, respiratory, vascular, and surgical complications are observed following RN. ${ }^{71}$

Despite similar reported rates of adverse events when comparing PN to RN, the importance of appropriate selection is critical in maintaining a low morbidity. Not all tumours are equally amenable to PN from a technical (surgical) standpoint. Careful preoperative assessment and selection should play into the decision-making process as specific tumour characteristics contribute to increased technical complexity and a greater likelihood of post-operative complication. Several methods of quantifying these tumours have been proposed as an objective means of pre-operative assessment and will play an 
increasing role in the future as urologists carefully weigh the risks and benefits of treatment decisions for SRMs. ${ }^{73-76}$

\subsubsection{Benefits of Nephron-sparing}

The most salient benefit of performing PN is the preservation of renal parenchyma and its subsequent benefits on long-term renal function. One of the initial studies demonstrating this relationship assessed surgical approach for the treatment of small, cortical renal tumours and the new onset of CKD using the Modification in Diet and Renal Disease Study (MDRD) equation to estimate GFR. RN was identified as a strong independent predictor for patients developing new-onset CKD (GFR $<60 \mathrm{ml} / \mathrm{min}$ and $<45 \mathrm{ml} / \mathrm{min}$ per $1.73 \mathrm{~m}(2))$. ${ }^{2}$ Many other retrospective studies assessing different populations and utilizing various measures of renal function validated these results..$^{67,77-79}$

Other studies have demonstrated the association between worsening renal function and increased risk of cardiovascular events and death. Go et al. laid the foundation for this work in their study analyzing over 1,000,000 patients from the Kaiser Permanente system in Northern California. They demonstrated a clear, step-wise relationship between worsening renal function and increasing risks of death and cardiovascular events. ${ }^{4}$ Much of this work has been performed on cohorts from the general population, not specifically patients with renal tumours. Such associations between PN and adverse cardiovascular outcomes and mortality were then extrapolated across populations.

Based on these assumptions, investigators then assessed whether or not this relationship could be established more convincingly by demonstrating a direct association 
between surgical approach and outcomes of death and cardiovascular events. ${ }^{3,79-81}$ Huang et al. utilized the SEER-Medicare database to assess patients with $\leq 4 \mathrm{~cm}$ tumours treated with either PN or RN between 1995 and 2002. RN was associated with an increased risk of mortality (hazard ratio (HR) 1.40) and a 1.4 times greater number of cardiovascular events. Overall 5-year survival was $74 \%$ after PN and $68 \%$ after RN. ${ }^{3} \mathrm{~A}$ more contemporary evaluation of the SEER-Medicare database assessed elderly patients with T1a tumours using an instrumental variable approach to control for differences between groups. Patients undergoing PN had a lower risk of death (HR 0.54, 0.34-0.85), which translated to a predicted increase in survival of $15.5 \%$ for those undergoing PN compared to $\mathrm{RN}$ at 8 years following treatment. ${ }^{79}$

The most recent controversy in this field revolves around the only randomized, controlled trial addressing these questions ${ }^{66}$ In this multicentre study published in 2011, 541 patients with small $(\leq 5 \mathrm{~cm})$ renal masses suspicious for RCC and a normal contralateral kidney were randomized to treatment with either PN or RN. With a median follow-up of 9.3 years, they identified better overall survival in patients undergoing RN compared to those undergoing PN. Intention-to-treat analysis demonstrated a 10-year overall survival rate of $81.1 \%$ for RN and $75.7 \%$ for PN (HR 1.50, 95\% CI 1.03-2.16). This conclusion contradicts the breadth of non-randomized literature demonstrating a benefit of nephron-sparing approaches on overall survival.

Although this represents the only randomized data in this field, debate persists regarding its findings. Certain aspects of this study should be approached with caution regarding its generalizability to a broader population. One of the major concerns is the timeframe of the study, which spans from 1992 to 2003. This spans a time period in 
which PN developed from an uncommon procedure with few indications to an accepted standard for small renal masses. During this period PN also transitioned from a developing technique with little expertise to a widely accepted procedure with few associated complications. Finally, the study reports on overall survival, but does not comment on other outcomes including renal function or cardiac morbidity, which are other important components of the decision making process.

On the other hand, findings from this trial offer important insight and information to complement the breadth of retrospective data. Perhaps the retrospective data are not as strong as initially perceived, especially given the potential biases present between treatment groups. The truth may lie somewhere in between, where certain patients will benefit more than others from PN depending on individual comorbidity and risk profile.

In summary, the majority of observational data supports the benefits of PN, though this recent randomized trial questions the strength of that association and the inherent biases of observational studies regarding the outcome of overall survival. However, outcome measures related to renal function and CKD are not reported in the trial and conclusions cannot be drawn in this regard. Such findings reiterate that many of these questions remain debatable and that further analysis is essential to identify which patients may be impacted the most by our surgical choices.

\subsection{Underutilization of Partial Nephrectomy}

\subsubsection{Overall rates}

Despite strong evidence supporting the use of PN, there has been a slow uptake of this procedure and a persistent overuse of RN. Several studies have demonstrated this 
trend at the population level. The most recent US population-level data report overall rates of PN use for all kidney tumours rising from $15 \%$ to $25 \%$ between 2002 and $2008 .^{82}$ The rate of PN use across population-level data specifically for T1a tumours has been reported as $42 \%$ in $2006^{58}$. Though rates of PN have steadily increased, they still fall below an ideal rate of utilization, particularly in small renal masses. Though not every tumour or patient is an ideal candidate for PN, experts have estimated that approximately $70 \%$ of patients presenting with T1a tumours have lesions for which it is technically feasible to perform PN and this procedure should thus be considered the standard of care for such patients. ${ }^{43}$

\subsubsection{High-risk Subgroups}

Certain subgroups are at the greatest risk of developing CKD and ultimately ESRD, and thus represent a high-risk population in which nephron-sparing approaches should be performed whenever possible. In turn, underutilization of PN in such groups represents an even greater concern than in the overall population of individuals with T1a tumours. Two of the most well-established risk factors for the development of CKD are diabetes mellitus and hypertension. Despite an elevated risk of CKD in these patients, initial evidence has demonstrated that we fail to preferentially perform $\mathrm{PN}$ in this population. Using Canadian population data, Abouassaly et al. demonstrated that rates of

PN utilization were equivalent regardless of preoperative morbidity and risk of CKD.${ }^{83}$ In particular, the odds ratio of undergoing a PN in the setting of HTN or diabetes was 1.07 (95\% CI 0.98-1.16) and 1.08 (95\% CI 0.96-1.20), respectively. The impact of the 
underutilization of PN may be most relevant and most detrimental in this high-risk population.

\subsubsection{Reasons for underuse}

The overuse of RN, and thus the underuse of PN, is likely due to the interaction of multiple contributing factors. One of the major issues may be the increased technical complexity of performing PN compared to RN. Fewer urologic surgeons have significant experience with this procedure, given that only recently has PN been considered a standard practice. Those with a greater experience likely represent younger surgeons who completed their training more recently or urologic surgeons who embraced PN early in its uptake. ${ }^{84-86}$ There is also a general perception of increased complication rates and shortterm morbidity associated with PN compared to RN. Current evidence supports a slightly different profile of complications, though the actual rates of overall complications are similar in most reported series. ${ }^{87}$

There is also evidence to suggest that the rapid uptake of laparoscopic RN may have had detrimental effects on the uptake of PN. Through their analysis of Canadian population-level data, Abouassaly et al. demonstrated an abrupt and significant decrease in the rate of uptake of PN coinciding with a rapid upswing in the uptake of laparoscopic RN. ${ }^{5}$ Though multiple factors likely contributed to this unexpected pattern of uptake, the end result was a preference for laparoscopic $\mathrm{RN}$ in a number of cases that may have otherwise been candidates for PN.

A final concern is that we still lack knowledge in specific areas to convincingly support PN over RN in the surgical treatment of SRMs in all patients. The vast majority 
of data, and the strongest evidence we have attained thus far, has focused on measures of renal function such as serum Creatinine and glomerular filtration rate (GFR). Up to this point, we have not established the relationship between surgical approach (PN vs. RN) and hard outcomes of ESRD such as dialysis or renal transplantation. The lack of such data may still provide a barrier to uptake for this procedure.

\subsection{Limitations in the Literature}

One of the major limitations of the body of literature surrounding arguments for PN versus $\mathrm{RN}$ in the surgical management of SRMs is establishing the relationship between surgical approach and hard outcomes of chronic kidney disease (dialysis or renal transplantation). CKD is graded based on GFR and although CKD is undesirable, surgically induced CKD may have a different natural history than that secondary to medical etiologies such as DM and/or HTN. Thus, ESRD requiring renal replacement therapy in patients treated for RCC is a more relevant measure.

The lack of such evidence may be a major contributing factor to explain the delayed uptake of this procedure. Perceptions remain that nephron-sparing approaches may not significantly impact long-term patient outcomes. Developing associations between surgical approach and both clear and clinically relevant outcomes may be necessary in this story. The absence of such associations will continue to detrimentally impact the diffusion and implementation of these practices in the future.

Furthermore, ESRD requiring renal replacement therapy represents an important outcome in and of itself. Chronic dialysis represents a major source of morbidity for the patient and a major source of health resource utilization. Mortality rates once a patient 
has been initiated on chronic dialysis have fallen over the years but have remained quite elevated at approximately 250 per 1000 patient years for those in their first year of dialysis. ${ }^{88}$ Mortality rates amongst those with CKD in the absence of ESRD have been reported at approximately 160 per 1000 patient-years, whereas death rates for those without CKD range from 50-100 per 1000 patient-years. ${ }^{89}$ These patients also suffer from complications and hospitalizations related to increased cardiac morbidity and infection rates. Rates of cardiovascular disease for those on dialysis were 1100 per 1000 patientyears compared to 550 for those with CKD in the absence of ESRD and 180 for those without CKD. Similar trends were reported regarding infection-related hospital admissions of 800 per 1000 patient-years for those on dialysis compared to 350 for those with CKD but not on dialysis and 100 for patients without CKD. ${ }^{89}$ Thus, CKD itself is associated with higher risks of cardiac morbidity, serious infections, and mortality, and CKD with ESRD is associated with even higher risks of all 3 outcomes. Moreover, there is a huge financial cost of maintaining a patient on dialysis with the annual cost of the treatment alone ranging from $\$ 26,000$ to $\$ 51,000$ (in the United States) depending on the specific modality. ${ }^{90}$

Over 3000 surgeries for renal tumours are performed in Canada yearly, and the majority of these consist of radical nephrectomies. Given this volume of cases, the potential burden of preventable ESRD following RN could have a significant impact at the population level (although actual estimates of preventable ESRD post RN are nonexistent). For these reasons, identifying the relationship between surgical approach and ESRD requiring renal replacement therapy remains a significant concern and major limitation in the current literature. In this thesis, the outcome of ESRD as a result of RN 
or PN is examined to address this important gap in knowledge. In addition, multiple methodolic approaches (Kaplan-Meier curves, Cox PH models, propensity score adjustment, and competing risks analysis) were utilized to reduce bias. 


\section{CHAPTER 3 METHODS}

\subsection{Study design}

A population-based, retrospective cohort study with data derived from linked administrative databases.

\subsection{Data Sources}

\subsubsection{Ontario Cancer Registry}

The Ontario Cancer Registry (OCR) is the largest and most comprehensive cancer database in the province of Ontario. It identifies virtually all pathologically confirmed malignant neoplasms in the province with the exception of non-melanoma skin cancer. There are four means by which patients are captured for entry into the OCR. These include the following: 1) Cancer-related hospital discharges and day surgeries obtained from the Canadian Institute for Health Information; 2) Cancer-related pathology reports; 3) Records from patients seen for treatment or consultation in one of the 14 regional cancer centres; 4) Death certificates obtained from the Ontario Registrar General with cancer identified as the primary cause of death. ${ }^{91}$

Re-abstraction studies have identified an ascertainment rate of 90-95\% for all malignancies in the province of Ontario. ${ }^{92}$ Other variables available within the OCR include basic demographic information, histology, date of diagnosis, cause of death, and date of death.

\subsubsection{Institute for Clinical Evaluative Sciences}


The Institute for Clinical Evaluative Sciences (ICES) was established in 1992 with the goal of providing information to help direct the future of the Ontario health care system. ICES targets anyone involved in such change including policy makers, physicians, administrators, and researchers. The tools by which ICES is able to facilitate these goals is a series of administrative databases stored within ICES. These databases are unique in their ability to link population-based health information at the level of the individual. Such linkages are performed with unique patient identifiers in a manner that ensures both privacy and confidentiality. ${ }^{93}$

The Ontario Cancer Data Linkage Project is an initiative within ICES meant to improve the accessibility of these data for researchers. This is a joint initiative with ICES and Cancer Care Ontario (CCO) specifically targeting cancer-related health services research in which de-identified information is provided to researchers on a cd (cd-link). ${ }^{91}$,

${ }^{93}$ Such an approach bypasses the need for a direct physical presence in ICES to retrieve the data, thus providing broader access. The specific databases utilized for this cd-link project are described below.

\subsubsection{Canadian Institute for Health Information}

The Canadian Institute of Health Information (CIHI) is a non-profit organization that collects, analyzes, and publishes data related to healthcare provided in the Canadian health system. The goals of CIHI are to provide information that is both comprehensive and standardized and that can be used to monitor, compare, and improve care. ${ }^{94}$

The specific subset of CIHI data that were utilized for this project is the CIHI Discharge Abstract Database (CIHI DAD). The CIHI DAD captures patient-level 
information from all acute care hospitalizations in the province of Ontario and throughout Canada. It specifically includes demographic, clinical, and administrative information from all acute inpatient and same-day surgery facilities. Each hospitalization is associated with an individual record. All information is received directly from the acute care facilities and provides access to clinical information on procedures performed, in-hospital complications, and patient comorbidities. Coding of diagnoses within this database used the International Classification of Diseases-9 (ICD-9) system prior to 2002 and the International Classification of Diseases-10 (ICD-10) from 2002 onward. The Canadian Classification of Diagnostic, Therapeutic, and Surgical Procedures (CCP - prior to January 2002) and Canadian Classification of Health Interventions (CCI - starting in 2002) were utilized for the coding of procedures. Re-abstraction studies are performed regularly by the CIHI Data Quality Department as a further means of ensuring quality of data and accuracy of coding. ${ }^{95-98}$

\subsubsection{Ontario Health Insurance Plan Database}

The Ontario Health Insurance Plan (OHIP) database encompasses all physician billing claims for insured services in the province of Ontario. In the setting of universal care, approximately $95 \%$ of Ontario physicians are in a fee-for-service practice and use OHIP billing claims for reimbursement. Each billing code is associated with information on the specific service provided (consultation or procedure), date of service, individual receiving the service, and primary diagnosis. ${ }^{99}$

\subsection{Linkages}


All datasets were linkable through ICES using unique patient identifiers (i.e. deterministic linkage). Specific data fields were selected from each database. These data fields were then extracted, linked across databases within ICES and provided as a specific cd-link dataset.

\subsection{Ethics and Confidentiality}

This study utilizes information obtained from administrative databases. Data were initially provided on a $\mathrm{CD}$, which was stored in a locked cabinet. Only authorized study personnel accessed data. Individual patient records were de-identified within ICES and linked with a unique identifier prior to being provided for research purposes. Following standard procedures as part of the cd-link data release, the datafile generated within ICES was subjected to privacy risk analysis, prior to release to the investigators, using proprietary software (PARAT) to ensure an acceptable level of risk $(<5 \%)$ of reidentification. No attempts were made to identify individual patients and all data were analyzed and presented in aggregate form. Ethics approval was obtained from the University of Toronto. A copy of the letter of approval from the Office of Research Ethics at the University of Toronto is shown in Appendix B.

\subsection{Dataflow}

The initial cohort, identified through the OCR, selected all patients with pathologically confirmed kidney cancer in the province of Ontario between the years 1995 and 2010. From this initial cohort, we identified patients who underwent surgical treatment and classified them as either having undergone $\mathrm{RN}$ or PN using $\mathrm{CCP} / \mathrm{CCI}$ 
procedure codes. Finally, the OHIP database was utilized to determine whether patients developed ESRD requiring renal replacement therapy (chronic dialysis or renal transplantation) (Figure 3.1). We had a look-back period of 2 years prior to the time of diagnosis for each patient in order to identify pre-operative comorbidities. A look forward period was available for all patients until the most recent available data update (March 2011).

Figure 3.1: Dataflow

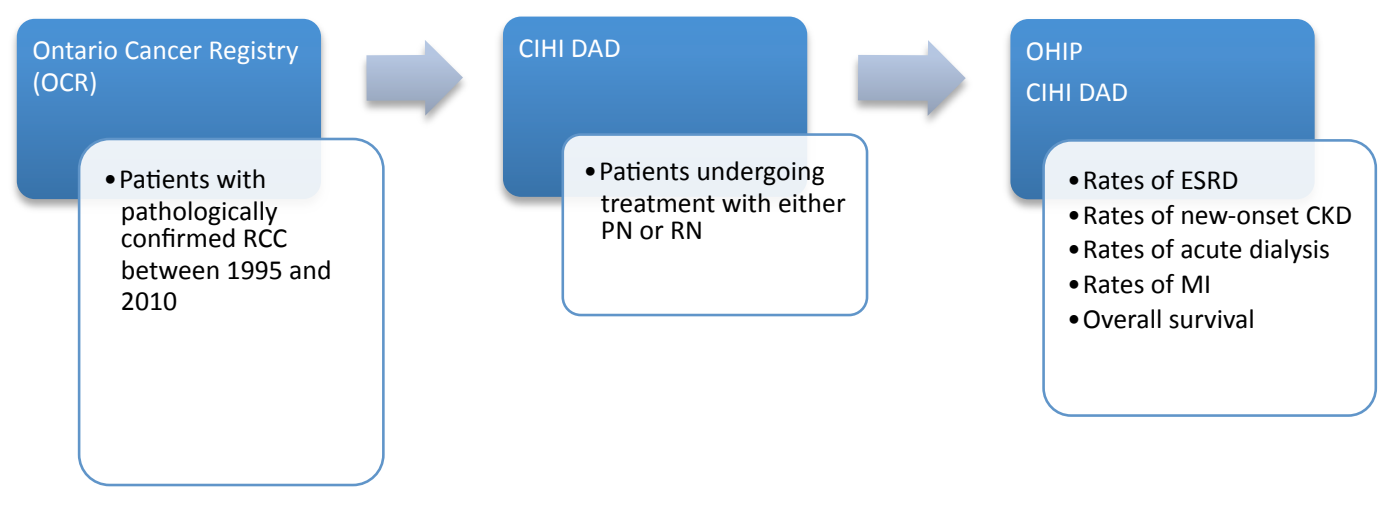

\subsection{Population (Inclusion/Exclusions)}

The cohort for this study was initially identified through the OCR and included all incident cases of pathologically confirmed kidney cancer in the province of Ontario between 1995 and $2011(n=22,188)$. Patients with either unspecified histology or with kidney malignancy other than RCC were excluded $(n=5,987)$. Patients who were not managed primarily with surgery $(\mathrm{RN}, \mathrm{PN})$ were excluded $(\mathrm{n}=3,062)$. The management of these excluded patients was not delineated in the current study. A percentage of these patients likely presented with advanced or metastatic disease and were managed 
primarily with systemic therapy. Other patients were likely managed with ablative therapies or active surveillance/watchful waiting approaches. Patients undergoing multiple renal surgeries between 1995 and 2010 were also excluded from the analysis. Further exclusions included patients with pre-operative ESRD requiring chronic dialysis $(\mathrm{n}=169)$ or renal transplantation $(\mathrm{n}=15)$. Also excluded were those who had previously undergone acute dialysis $(\mathrm{n}=11)$. Those with a prior diagnosis of a non-kidney malignancy (i.e. second tumour) were excluded $(\mathrm{n}=872)$. Finally, patients with histologic sarcomatoid differentiation were excluded $(\mathrm{n}=135)$. This was because patients with sarcomatoid histology represented a subset of patients with particularly aggressive disease whose behaviour was distinctly different than the remainder of the cohort. Exclusion of such patients was considered a priori based on prior literature ${ }^{100}$, however such findings were confirmed within our own dataset and patient population prior to making such a decision. Review of the Ontario population confirmed these findings with a 5-year cancer-specific survival (CSS) of $41 \%$ for those with sarcomatoid pathology compared to $65 \%$ for those without sarcomatoid differentiation. The final cohort consisted of 11, 937 individuals (Figure 3.2). 
Figure 3.2: Flowchart for identification of final cohort

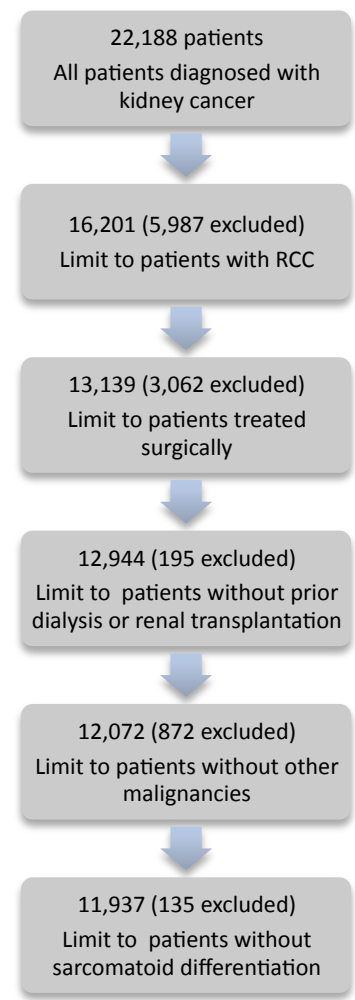

\subsection{Covariables}

\subsubsection{Demographic data}

The OCR provides information on patient gender and age. Gender was analyzed as a dichotomous variable (male, female). Patient age was determined at the time of diagnosis and analyzed as a categorical variable $(<50,50-59,60-69,70-79, \geq 80$ years old). Year of surgery was obtained from the CIHI DAD and was analyzed both as a continuous and categorical (1995-1999, 2000-2004, 2005-2010) variable. Year of surgery was assessed as a categorical variable in our final analysis as these time intervals were felt to be more clinically meaningful while maintaining the ability to appropriately capture the trends. Within the CIHI database, the accuracy of demographic data has been demonstrated to range from $93-100 \%$ when compared to the hospital record as the gold standard. $^{95-98,101,102}$ 


\subsubsection{Renal cell carcinoma}

Patients with renal cell carcinoma (RCC) were identified using ICD-9 and ICD-10 diagnostic codes (Table 3.1). ICD-9 coding was utilized until 2002, at which point coding schemes were updated to ICD-10. Patients with urothelial carcinomas of the renal pelvis or ureter were specifically identified and excluded based on ICD-9 $(189.1,189.2)$ and ICD-10 (C65.9, C66.9) diagnostic codes. Information on specific histologic subtype was also available through the OCR and allowed further classification of RCC tumors as clear cell, papillary, chromophobe, sarcomatoid, or unspecified.

Table 3.1: ICD-9/10 diagnostic codes for renal cell carcinoma

\begin{tabular}{|l|l|}
\hline Renal cell carcinoma & Codes \\
\hline ICD-9 & 189.0 (malignant kidney neoplasm, except renal pelvis) \\
\hline ICD-10 & C64.9 (malignant kidney neoplasm, except renal pelvis) \\
\hline
\end{tabular}

\subsubsection{Surgery (PN/RN)}

All patients undergoing surgical treatment for RCC were identified using the CIHI DAD. CCP and CCI procedure codes were utilized to classify patients as having undergone RN or PN (Table 3.2). Studies specifically assessing the validity of procedural coding for RN or PN have not been performed. However, other surgical procedures have been validated using the medical record as a gold standard. These have included urologic procedures such as radical prostatectomy, transurethral resection of the prostate and 
bilateral orchiectomy, which were demonstrated to have almost complete agreement (kappa $0.86-0.94) .{ }^{103}$ Procedural codes for knee replacement surgery were validated in the CIHI database and compared to the hospital record from 5 Ontario hospitals with a greater than $94 \%$ complete agreement and a false positive rate of $<5 \%$. ${ }^{102}$

Table 3.2: CCP/CCI procedural codes for radical and partial nephrectomy

\begin{tabular}{|l|l|}
\hline Radical nephrectomy & Codes \\
\hline Open & CCI: 1PC89/91LB, 1PC89/91PF, 1PC89/91QF \\
\hline & CCP: 6741, 6742, 6744 \\
\hline Laparoscopic & CCI: 1PC91DA, 1PC89DA, 1PC91AB \\
\hline Partial nephrectomy & \\
\hline Open & CCI: 1PC87LA, 1PC87LAXXE, 1PC87LAXXG, 1PC87NQ \\
\hline Laparoscopic & CCI: 1PC87DA \\
\hline
\end{tabular}

\subsubsection{Chronic dialysis}

Physician billing codes from the OHIP database were used to identify patients undergoing chronic dialysis (Table 3.3). A minimum of 12 billing codes for chronic dialysis (billed weekly) over a 6-month period was required to meet criteria for classification as requiring chronic dialysis treatment. A binary outcome variable was then created based on this algorithm. Quinn et al. assessed the validity of this algorithm in a multicentre chart abstraction study in Ontario. An incident dialysis population was evaluated between the years 2001 and 2005 using the medical record as the reference standard. Patients were correctly identified as having received outpatient dialysis with a 
sensitivity of $100 \%$ and a positive predictive value of $96 \%$. They identified an $88 \%$ agreement for treatment initiation date within a 28-day window, as well as strong agreement in coding for secondary variables including demographic data (age, sex), initial dialysis modality (96.1\% agreement), death ( $95.6 \%$ agreement), and subsequent renal transplantation $(99.1 \%$ agreement $) .{ }^{104}$

Table 3.3: OHIP billing codes for acute and chronic dialysis

\begin{tabular}{|l|l|}
\hline Chronic dialysis & Codes \\
\hline Hemodialysis (OHIP) & G326, G860, G333, G862, G863, G865, G866 \\
\hline Peritoneal dialysis (OHIP) & G332, G333, G861, G864 \\
\hline Acute dialysis & \\
\hline Hemodialysis (OHIP) & R849, G323 \\
\hline Peritoneal dialysis (OHIP) & G330, G331 \\
\hline
\end{tabular}

\subsubsection{Acute dialysis}

Physician billing codes from the OHIP database were used to identify patients undergoing acute dialysis (Table 3.3). A single physician billing code was considered sufficient for classification as having undergone acute dialysis. Acute dialysis was then analyzed as a binary time-to-event outcome. The validity of acute dialysis coding in the CIHI database has not been specifically assessed. However, American studies have assessed the validity of acute dialysis coding (ICD codes) in identifying those with acute renal failure with a sensitivity of $90 \%$ and positive predictive value of $94 \% .{ }^{104,105}$ 


\subsubsection{Renal transplantation}

Renal transplantation was identified through the CIHI DAD using CCP/CCI procedure coding (Table 3.4). The validity of procedural coding within the CIHI database has previously been demonstrated to have a high degree of agreement when compared to the medical record. Procedural coding in general has been reported at greater than $94 \%$ for complete agreement with false positive rates of $<5 \%$. When only the primary procedure during a hospitalization is evaluated, complete agreement is reported in greater than $99 \%$ of cases. ${ }^{102,106}$

Table 3.4: CCP/CCI procedural codes for renal transplantation

\begin{tabular}{|l|l|}
\hline Renal transplantation & CCP: 6750, 6759 \\
\hline & CCI: 1PC85LAXXJ, 1PC85LAXXK \\
\hline
\end{tabular}

\subsubsection{Renal Replacement}

The primary outcome measure of ESRD requiring renal replacement therapy was a combined outcome of having received either chronic dialysis or renal transplantation. A dichotomous outcome variable was then created utilizing the previously described coding and algorithms for chronic dialysis and renal transplantation. Patients who required acute dialysis in the post-operative period and subsequently progressed to ESRD remained eligible for the acquiring the outcome of ESRD.

\subsubsection{Comorbidity Adjustment}


Comorbidity adjustment is important in any retrospective study due to issues of confounding. They are particularly important for our question because strong issues of selection bias may occur with our patients. Also, there are very distinct comorbidities that may drive treatment selection and directly impact renal functional outcomes. These include DM, HTN, and prior CKD. Because of their importance, we analyzed several methods of controlling for comorbidity. Each method offered its own strengths and weaknesses and we wanted to compare them to ensure a stronger analysis. We ultimately chose to analyze with individual comorbidities because we felt it better adjusted for variables in the groups that we believed were the driving forces behind selection of type of surgery.

\section{Charlson-Deyo comorbidity score}

The Charlson comorbidity index is calculated based on 17 distinct categories of comorbidity (e.g. myocardial infarction) that are summed and comprise a total summary score. Following convention, the category of "malignancy" was removed prior to calculation in this study. Summary scores were then collapsed into a categorical variable based on score distribution with the following categories: $0,1,2+$.

The Charlson comorbidity index is a well validated and widely used method of assessing comorbidity and predicting outcomes. It was initially developed as a method of predicting mortality based on comorbid disease and has since been applied to administrative databases using ICD-9 diagnostic coding as the Charlson-Deyo comorbidity index. ${ }^{107,108}$ It has also since been adapted to ICD-10 coding and has demonstrated both good performance and prediction. ${ }^{109,110}$ Numerous studies in the 
literature have utilized the Charlson-Deyo comorbidity index as a reliable means of controlling for comorbidity, and it has been well correlated with post-operative complications and mortality in both cancer and non-cancer settings. ${ }^{106,111}$ The Charlson comorbidity index has also been associated with mortality in the setting of kidney cancer patients ${ }^{112}$, as well as with blood transfusion rates in patients undergoing nephrectomy for renal masses. ${ }^{113}$

\section{Johns Hopkins Adjusted Clinical Groups System}

The Johns Hopkins Adjusted Clinical Groups (ACGs) system utilizes an algorithm based on ICD-9 and ICD-10 diagnostic codes to characterize a patient's illness. It is a unique proprietary system that utilizes both inpatient and outpatient diagnoses in its scoring. Each ICD-9 and ICD-10 diagnosis is assigned to 1 of 32 Aggregated Diagnosis Groups (ADGs). Individuals are then assigned to 1 of 106 ACGs, with the assumption that individuals within the same ACG will have similar health resource utilization. ADGs can also be collapsed into one of 12 Collapsed ADGs (CADGs). ${ }^{114}$

CADG and ADG scores were utilized in the current study in 2 methods. First a sum of the 12 CADGs was obtained and analyzed in the following categories: 0-2, 3, 4, 5, $6,7,8,9+$. Secondly, ADGs were analyzed using a previously validated scoring system utilizing weighted ADG scores and collapsing age and gender (Mortality Risk Score). ${ }^{115}$

The Johns Hopkins ACG system has been well established as a tool to predict health care resource utilization, but more recent studies have also demonstrated its ability to predict mortality and long-term hospitalization. ${ }^{116}$ Furthermore, ADGs accurately predict 1-year mortality in the general population and performed well compared to other established methods of comorbidity adjustment (Charlson comorbidity score). ${ }^{117}$ The 
Mortality Risk Score has also proved to be a strong predictor of 1-year mortality and a useful tool for comorbidity adjustment. ${ }^{115}$ A distinct advantage of the Johns Hopkins ACG system over the Charlson comorbidity score is that it utilizes both inpatient and outpatient health administrative data, whereas the Charlson score relies solely on inpatient diagnoses.

\section{Diabetes mellitus}

Those with diabetes mellitus (DM) were identified using an algorithm requiring either a single hospitalization discharge (CIHI DAD) with a diagnostic code for DM or 2 physician billings claims (OHIP) with a diagnosis of DM within a 2-year period. (Table 3.5) This algorithm has been well validated in the province of Ontario and is utilized to identify patients for inclusion in the Ontario Diabetes Database. This algorithm was originally developed and validated using claims data in the province of Manitoba with a sensitivity greater than $95 \% .{ }^{118}$ This algorithm was validated within Ontario administrative databases by Hux et al. who demonstrated $86 \%$ sensitivity, $97 \%$ specificity, and $80 \%$ positive predictive value in identifying diabetic patients. The validity of this algorithm in capturing diabetics within administrative databases was compared against the Ontario Drug Benefit Program (claims for insulin or oral hypoglycemics medications), as well as results from a self-reported health survey. ${ }^{118}$

\section{Hypertension}

Those with hypertension were identified using an algorithm of 2 outpatient physician billing claims (OHIP) with a diagnosis of hypertension within a 2-year period 
or a single inpatient diagnostic code (Table 3.5). A similar algorithm allowing for a 3year capture period has previously been utilized and validated in the literature. This algorithm was modified for this study due to the fact that only a 2-year look-back period of data was available to identify pre-operative comorbidity. A diagnosis of pre-operative hypertension was also allowed if the requirements of the algorithm were met and at least the initial diagnosis of hypertension occurred prior to surgery. This algorithm was validated in an Ontario-wide study demonstrating a sensitivity of $73 \%$, specificity of $95 \%$ and positive predictive value of $87 \%$ when compared to physician-assigned diagnoses within the medical record. ${ }^{119}$

\section{Myocardial infarction}

Patients who have had a myocardial infarction (MI) were identified through an acute inpatient hospitalization (CIHI DAD) with a most responsible diagnosis of MI according to ICD-9 or ICD-10 diagnostic codes (Table 3.5). In addition, their hospitalization had to be a minimum of 3 days. This current algorithm was utilized to develop predictive models for mortality within the Ontario population and has been further validated with the new ICD-10 coding. ${ }^{120,121}$ Accuracy of this diagnosis when compared to the hospital chart was approximately $94 \% .{ }^{122}$ Other studies validating CIHI diagnostic codes for MI demonstrated a sensitivity of $89 \%$ and positive predictive value of $89 \% .{ }^{123}$ This current algorithm has been utilized in multiple other studies and has been widely adopted in the literature. ${ }^{124,125}$ Myocardial infarction was analyzed as a dichotomous variable and utilized as both a pre-operative comorbidity and a postoperative outcome. Patients with a pre-operative MI were excluded from analysis for the 
outcome of post-operative MI. Otherwise, these patients were included in the analysis and pre-operative MI was accounted for in the analysis of comorbidity. Patients with peri-operative MI during the same hospitalization as their surgery were not captured as a distinct MI event.

\section{Chronic kidney disease}

Those with chronic kidney disease (CKD) were identified using an algorithm that required either a single hospitalization discharge (CIHI DAD) with a diagnostic code for CKD or 2 physician billings claims (OHIP) with a diagnosis of CKD within a 3-year period (Table 3.5). In addition, outpatient physician billing claims had to be provided by either a primary care physician or nephrologist (Physician specialty code 00,13 , or 16).

Both methods of capture for patients with CKD have been analyzed previously. ${ }^{126}$ Lee et al. analyzed diagnostic codes for CKD within the CIHI database by comparing it to the medical record. Individuals with preexisting CKD based on inpatient diagnostic codes had a median serum Creatinine level of $2.96 \mathrm{mg} / \mathrm{dL}$ ( $262 \mathrm{umol} / \mathrm{L}$ ), while those without a diagnosis of CKD had a median serum Creatinine of $1.11 \mathrm{mg} / \mathrm{dL}(98 \mathrm{umol} / \mathrm{L})$. Data from the same study demonstrated a sensitivity of $45 \%$, specificity of $97 \%$, and positive predictive value of $72 \% .{ }^{127} \mathrm{CIHI}$ diagnostic codes in general have demonstrated high agreement for both primary (81-95\%) and secondary (88-99\%) diagnoses. ${ }^{128}$ The OHIP physician billings claims and associated diagnostic code for CKD were compared to the medical record and demonstrated a sensitivity of $74 \%$, specificity of $99 \%$, positive predictive value of $29 \%$, and negative predictive value of $99 \% .{ }^{129}$ 
Both criteria were incorporated in the identification of patients with CKD with a goal of achieving a more complete capture of CKD. CKD was analyzed as a dichotomous variable and assessed as both a pre-operative comorbidity and a post-operative outcome.

Table 3.5: ICD9/10 diagnostic codes for various comorbidities

\begin{tabular}{|l|l|}
\hline Diabetes mellitus & ICD-9: 2500 - 2509 \\
\hline Hypertension & ICD-10: E10 - E14 \\
\hline & ICD-9: 401-405 \\
\hline Myocardial infarction & ICD-9: 4100 \\
\hline Chronic kidney disease & $\begin{array}{l}\text { ICD-9: 582, 5820-5829,583, 5830-5839, 585, 5850, 586, } \\
5860,588,5880-5889\end{array}$ \\
\hline & ICD-10: N18, N19 \\
\hline
\end{tabular}

\subsection{Statistical Analysis}

\subsubsection{Power calculation}

We expected a total of 11,000 cases of surgically treated RCC eligible for analysis in this study. The rate of PN in Ontario during the relevant time-frame was approximately $20 \%$. The accrual period was 15 years and median follow-up expected to be 7 years. An estimated median time to event for our primary outcome (ESRD) was 10 years.

Therefore, with a Type 1 error probability (alpha) of 0.05 and an expected hazard ratio of 0.8 , we anticipated a power of 0.82 to be able to detect a hazard ratio of 0.8 or lower for ESRD associated with PN compared to RN. ${ }^{130}$ 


\subsubsection{Data checks}

Multiple checks were performed to ensure completeness and appropriateness of the data. Distribution of variables between treatment groups as well as its consistency with prior studies was assessed. Outcome variables were assessed between groups and over time to determine appropriate distributions and fluctuations across the years. Multiple cross-tabulations were performed to ensure that the data appeared logical across age groups, gender, and year of treatment.

\subsubsection{Descriptive analyses}

General descriptive statistics were calculated for the overall cohort and between the treatment groups. We assessed demographic, pathologic, and clinical variables. Continuous variables were expressed as means with standard deviations and compared between treatment groups (PN or RN) using a non-paired student's t-test. Dichotomous and categorical variables were expressed as percentages and compared between treatment groups using the Pearson's chi-square test. Significance was defined as a two-sided pvalue less than 0.05 . Overall rates of the primary and secondary outcomes were calculated for the entire cohort and also compared between treatment groups. Trends in choice of surgical treatment were reported across the years. No adjustment was made for multiple significance testing. ${ }^{131}$

\subsubsection{Cox proportional hazards model}

Time-to-event analysis was performed assessing ESRD requiring RRT as the primary outcome. Secondary outcomes (new-onset CKD, myocardial infarction, acute 
dialysis, overall survival, and cancer-specific survival) were also measured using time-toevent analysis. The main treatment variable (surgical approach) as well as each of the potential covariables were analyzed initially in a univariable Cox Proportional Hazards model to assess for statistically significant relationships with each of the outcomes. A multivariable model was then developed to determine the adjusted association between surgical approach and the various outcomes. Because of the large number of patients in our cohort, we were able to include all variables deemed clinically relevant (regardless of p-value in the univariate analysis) without concern for overfitting our model, following the guideline of a minimum of 10 events per variable. ${ }^{132}$ The proportional hazards assumption was tested graphically using log-log plots.

Various models were developed using different methods of assessing comorbidity and compared to one another. These included models using each of the summary comorbidity scores (ADG, Charlson) and also utilizing individually calculated comorbidities (DM, HTN, MI, CKD). Other covariables utilized in the multivariable model included demographic factors, disease and tumor characteristics, and year of procedure. Predictive models utilizing individually calculated comorbidity were ultimately used as the primary analysis because of their ability to discriminate between particularly relevant specific pre-operative clinical comorbidities (i.e. HTN, DM, CKD). Hazards ratios and $95 \%$ confidence intervals were provided for variables in each analysis using the Cox Proportional Hazards model. Significance was defined as a p-value less than 0.05 . Kaplan Meier curves were generated for each of the primary and secondary outcomes and were stratified by treatment choice. Curves were then compared for statistical difference utilizing the log-rank test. 


\subsubsection{Propensity score model}

Propensity scores were utilized as another method to reduce bias and to control for differences between treatment groups. We first determined propensity scores or the probability that a patient will receive a certain treatment ( $\mathrm{PN}$ or $\mathrm{RN})$ based on known pretreatment variables. Propensity scores were then included in predictive models to control for bias between groups and develop more balanced comparisons. Propensity scores for undergoing PN were obtained using a multivariable logistic regression model. The following variables were included in the model: age group, sex, year of surgery, histology, prior hypertension, prior MI, prior DM, prior CKD. The propensity scores were then divided into quintiles for further analysis. Variables were compared between the treatment groups within each quintile to ensure balance. (appendix A, Table 1)

Cox Proportional Hazards models were then used to assess the association between surgical approach and our various outcomes while controlling for propensity score as either a continuous and categorical (quintiles) variable (in separate models). Effect of surgical approach was presented as a Hazards Ratio with 95\% confidence intervals. Significance was defined as a p-value less than 0.05 .

Other methods of utilizing propensity score to control for confounding were considered. The most common of these alternate methods is utilizing propensity score to create a matched analysis. The potential benefit of such a strategy is the elimination from analysis of patients at the extremes, and it also avoids assumptions of a linear relationship between the propensity score and the outcome. It may also be advantageous when dealing with relatively rare outcomes or small sample sizes. Such methods, however, have 
potential disadvantages. A matched analysis will exclude all unmatched patients, which may not necessarily be appropriate and whose real benefit remains unknown. ${ }^{133}$

\subsubsection{Competing risks analysis}

There were concerns of possible competing risks impacting the validity of the results of the modeling approaches detailed above. The primary competing risk of concern in this cohort was overall mortality. The cohort of patients diagnosed with RCC represents a relatively frail and elderly population. In such situations, other failures (i.e. mortality) may occur prior to the outcome of interest (ESRD), thus obscuring the true impact of a selected intervention (such as PN). Such competing risks may represent a significant bias in this population given the overwhelming prevalence of mortality compared to rates of those progressing to ESRD. Prior studies have reported this in patients with medical renal disease and have been consistent with the current cohort ${ }^{4}$, in which the mortality rate was $35 \%$ at 5 years compared to a rate of ESRD of $2 \%$ at 5 years.

Cumulative incidence curves were obtained for each of the primary and secondary outcomes (other than overall survival), which were then analyzed factoring in the competing risk of death. A local expert in competing risks analysis (Melania Pintilie, $\mathrm{PhD}$ ) was consulted for assistance during this portion of the analysis, and the previously described SAS procedures "compcif", "compcp", and "cuminc" were utilized to perform this analysis. ${ }^{134}$ Cumulative incidence curves were then created and compared between 
the treatment groups utilizing the Pepe-Mori test. Statistical significance was set at a pvalue of less than 0.05 .

\subsubsection{Subset Analysis - Modern cohort}

A separate subset analysis was performed on a modern cohort of patients diagnosed between 2003 and 2010. Although this analysis was not planned a priori, as we proceeded with our analyses and gained a deeper understanding of the field, it became a critical analysis in understanding the impact of surgical approach on current, modernday practice. Indications for PN have shifted over time and the procedure has gained acceptance as a standard approach for the surgical management renal tumours only in more recent years. Major published series demonstrating oncologic equivalence between $\mathrm{RN}$ and PN in appropriately selected patients with T1a tumours were published around the year 2000. ${ }^{1,32,65}$ Many of the major studies demonstrating benefit for PN compared to $\mathrm{RN}$ regarding the preservation of renal function followed later, with the often referenced Huang study not appearing until $2006^{2}$. Furthermore, PN was not integrated into guidelines as the standard of care until 2009 with the publication of guidelines for T1 renal tumours by the American Urologic Association. ${ }^{43}$

Practice patterns and trends in uptake of PN demonstrated a slow dissemination and poor utilization prior to 2003. Studies utilizing administrative databases to assess national (US) trends in the utilization of PN for treatment of kidney tumours report overall rates of PN of less than $10 \%$ between the years of 1998 and $2002 .{ }^{135,136}$ Furthermore, utilization of PN was concentrated in high volume and teaching institutions during this time period. ${ }^{85}$ Dissemination and uptake progressed after 2002, and reflected a 
shift in practice patterns where PN was utilized for patients with normal contralateral kidneys and normal renal function as opposed to being reserved only for patients with solitary kidneys and impending renal failure. The impact of surgical technique on each of our primary and secondary outcomes was analyzed separately for the modern cohort to assess for differences in outcomes in a cohort that may be a more accurate reflection of current practice and patient selection. Baseline differences between the modern cohort and the earlier cohort were obtained. In addition, sensitivity analyses were performed to confirm findings including an analysis of sequential start dates for defining the modern period to ensure consistency of results. 


\section{CHAPTER 4 RESULTS}

\subsection{Descriptive Statistics}

A total of 11,937 patients were surgically treated for RCC in Ontario between 1995 and 2010 and met criteria for inclusion in the final cohort. A flow diagram of steps to obtain the final cohort is described in Figure 3.2. A total of 9,830 patients $(82 \%)$ underwent RN compared to 2,107 patients (18\%) who underwent PN. Baseline characteristics of patients within each treatment group and for the overall cohort are described in Table 4.1. Median follow-up was 59 months, with a median follow-up of 60 months amongst those undergoing $\mathrm{RN}$, compared to 48 months amongst those undergoing PN. Overall survival at 5 years was $65 \%$, with a 5-year overall survival of 59\% amongst those undergoing $\mathrm{RN}$, compared to $90 \%$ among those undergoing PN. Male patients comprised $60 \%$ of the cohort undergoing RN compared to $63 \%$ of those undergoing PN. Unspecified RCC (59\%) represented the most commonly diagnosed histologic subtype of RCC followed by clear cell histology (31\%). Half of all patients (48\%) were diagnosed in the most recent time frame (2005-2010) with $72 \%$ of all PNs having been performed during this period compared to $43 \%$ of all RNs. A pre-operative diagnosis of hypertension, diabetes mellitus, and CKD was present in $42 \%, 19 \%$, and $5 \%$ of patients, respectively. 
Table 4.1: Baseline characteristics

\begin{tabular}{|l|l|l|l|}
\hline & $\begin{array}{l}\text { Radical } \\
\text { nephrectomy }\end{array}$ & $\begin{array}{l}\text { Partial } \\
\text { nephrectomy }\end{array}$ & Overall \\
\hline Patients & $9,830(82 \%)$ & $2,107(18 \%)$ & 11,937 \\
\hline Median follow-up (mo) & $60(30-107)$ & $48(24-72)$ & $59(24-108)$ \\
\hline Sex & & & \\
\hline Male & $5,900(60 \%)$ & $1,335(63 \%)$ & $7,235(61 \%)$ \\
\hline Female & $3,930(40 \%)$ & $772(37 \%)$ & $4,702(39 \%)$ \\
\hline Age group & & & \\
\hline <50 & $1,787(18 \%)$ & $540(26 \%)$ & $2,327(19 \%)$ \\
\hline $50-59$ & $2,408(25 \%)$ & $592(28 \%)$ & $3,000(25 \%)$ \\
\hline $60-69$ & $2,704(28 \%)$ & $539(26 \%)$ & $3,243(27 \%)$ \\
\hline $70-79$ & $2,264(23 \%)$ & $383(18 \%)$ & $2,647(22 \%)$ \\
\hline$\geq 80$ & $667(7 \%)$ & $53(3 \%)$ & $720(6 \%)$ \\
\hline Histology & & & \\
\hline Clear cell & $2,966(30 \%)$ & $757(36 \%)$ & $3,723(31 \%)$ \\
\hline Papillary & $499(5 \%)$ & $217(10 \%)$ & $716(6 \%)$ \\
\hline Chromophobe & $371(4 \%)$ & $131(6 \%)$ & $502(4 \%)$ \\
\hline NOS & $5,994(61 \%)$ & $1,002(48 \%)$ & $6,996(59 \%)$ \\
\hline Year group & & & \\
\hline $1995-1999$ & $2,589(26 \%)$ & $76(4 \%)$ & $2,665(31 \%)$ \\
\hline $2000-2004$ & $2,972(30 \%)$ & $518(25 \%)$ & $3,490(29 \%)$ \\
\hline $2005-2010$ & $4,269(43 \%)$ & $1,513(72 \%)$ & $5,782(48 \%)$ \\
\hline Weighted ADG (quintile) & & & \\
\hline 1 (least comorbidity) & $1,921(20 \%)$ & $396(19 \%)$ & $2,317(19 \%)$ \\
\hline 2 & $2,326(24 \%)$ & $508(24 \%)$ & $2,834(24 \%)$ \\
\hline 3 & $2,054(21 \%)$ & $437(21 \%)$ & $2,491(21 \%)$ \\
\hline 4 & $1,712(17 \%)$ & $360(17 \%)$ & $2,072(17 \%)$ \\
\hline 5 & $1,817(18 \%)$ & $406(19 \%)$ & $2,223(19 \%)$ \\
\hline Charlson score & & & \\
\hline 0 (least comorbidity) & $7,471(76 \%)$ & $1,585(75 \%)$ & $9,056(76 \%)$ \\
\hline 1 & $1,860(19 \%)$ & $409(19 \%)$ & $2,269(19 \%)$ \\
\hline$\geq 2$ & $499(5 \%)$ & $113(5 \%)$ & $612(5 \%)$ \\
\hline Hypertension & & & \\
\hline No & $1,210(59 \%)$ & $1,210(57 \%)$ & $6,975(58 \%)$ \\
\hline Yes & $4,065(41 \%)$ & $897(43 \%)$ & $4,962(42 \%)$ \\
\hline Diabetes & & & \\
\hline No & $8,021(82 \%)$ & $1,651(78 \%)$ & $9,672(81 \%)$ \\
\hline Yes & $1,809(18 \%)$ & $456(22 \%)$ & $2,265(19 \%)$ \\
\hline CKD & $9,415(96 \%)$ & $1,927(91 \%)$ & $11,342(95 \%)$ \\
\hline No & $415(4 \%)$ & $180(9 \%)$ & $595(5 \%)$ \\
\hline Yes & & & \\
\hline Myocardial infarction & $9,725(98.9 \%)$ & $2,090(99.2 \%)$ & $11,815(99 \%)$ \\
\hline No & & & \\
\hline & & & \\
\hline & & & \\
\hline
\end{tabular}


\begin{tabular}{|c|l|l|l|}
\hline Yes & $105(1.1 \%)$ & $17(0.8 \%)$ & $122(1 \%)$ \\
\hline Abbreviations: $\mathrm{mo}=$ months; NOS = not otherwise specified; ADG = Johns Hopkins'
\end{tabular} Aggregated Diagnosis Groups; $\mathrm{CKD}=$ chronic kidney disease

\subsubsection{Data Checks}

Several assessments of the dataset were performed and general trends were examined to ensure that the data appeared appropriate and consistent with previously established figures. The number of surgeries performed for the treatment of RCC in the province of Ontario increased steadily between 1995 and 2010, with 1,047 such surgeries being performed in 2010. Rates of PN in the province increased steadily and peaked at a rate of $33 \%$ in 2010, the most recent year of the cohort (Figure 4.1).

Figure 4.1: a) Numbers of cases of PN and RN performed in the province of Ontario (1995-2010); b) Percentage of cases performed as either RN or PN by year in the province of Ontario.

a)

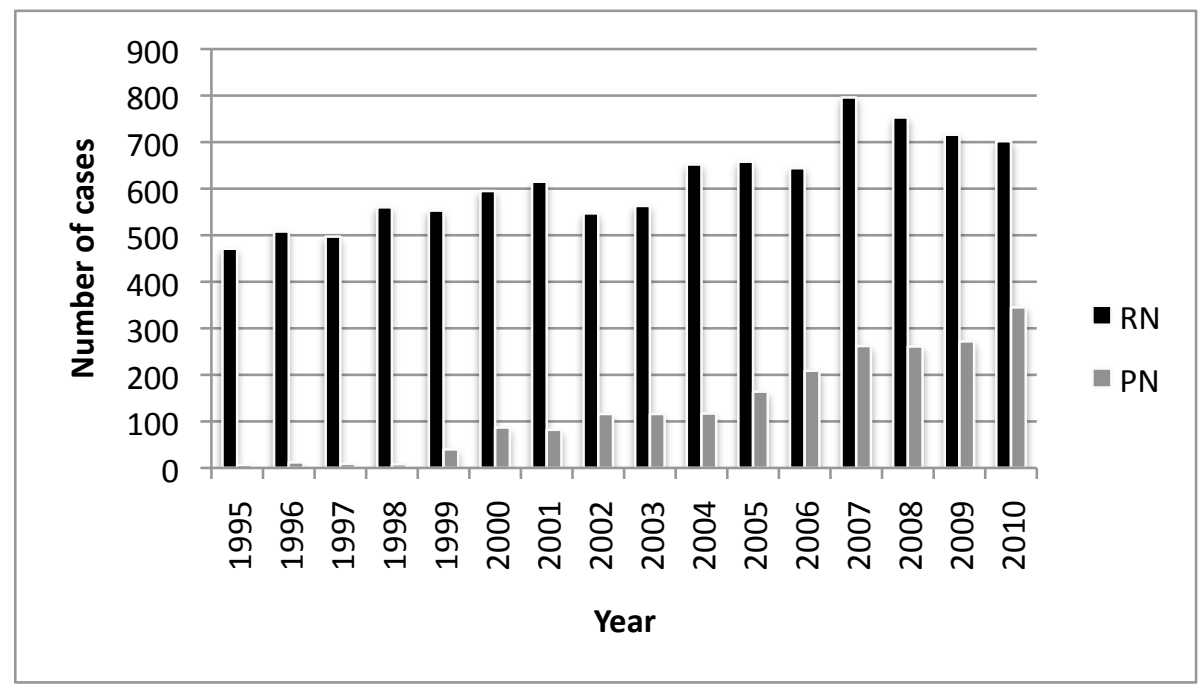


b)

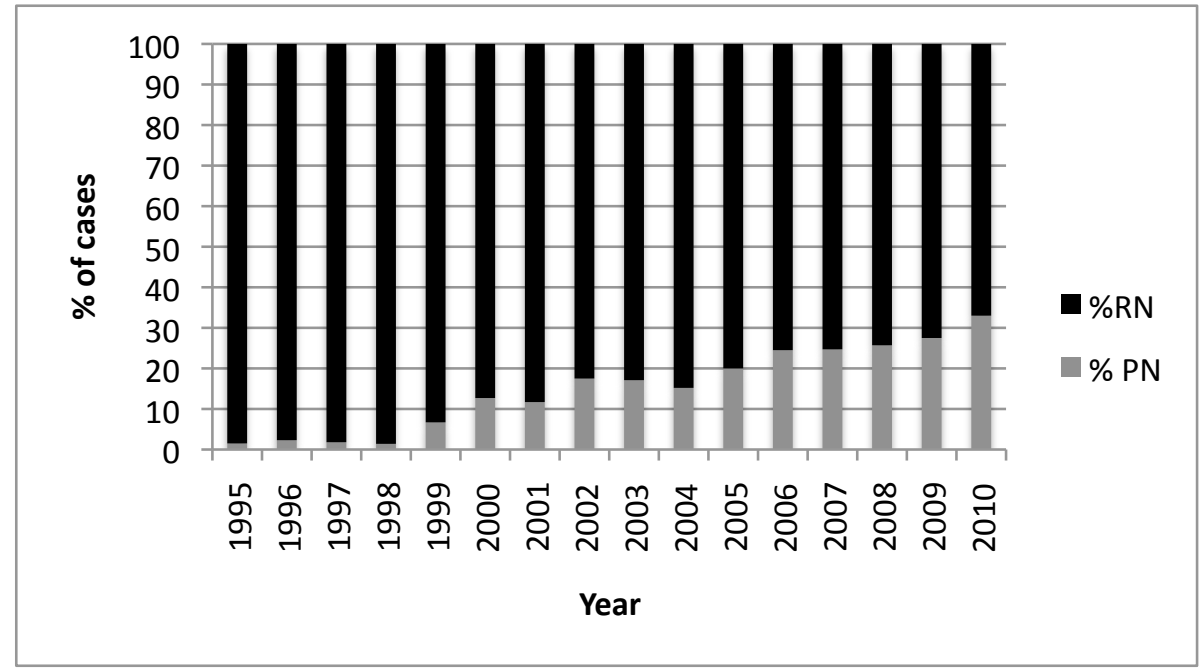

Rates of renal replacement therapy demonstrated a peak in the initial quarter following surgery and rapidly declined (Figure 4.2).

Figure 4.2: Absolute number of ESRD events by post-operative quarter

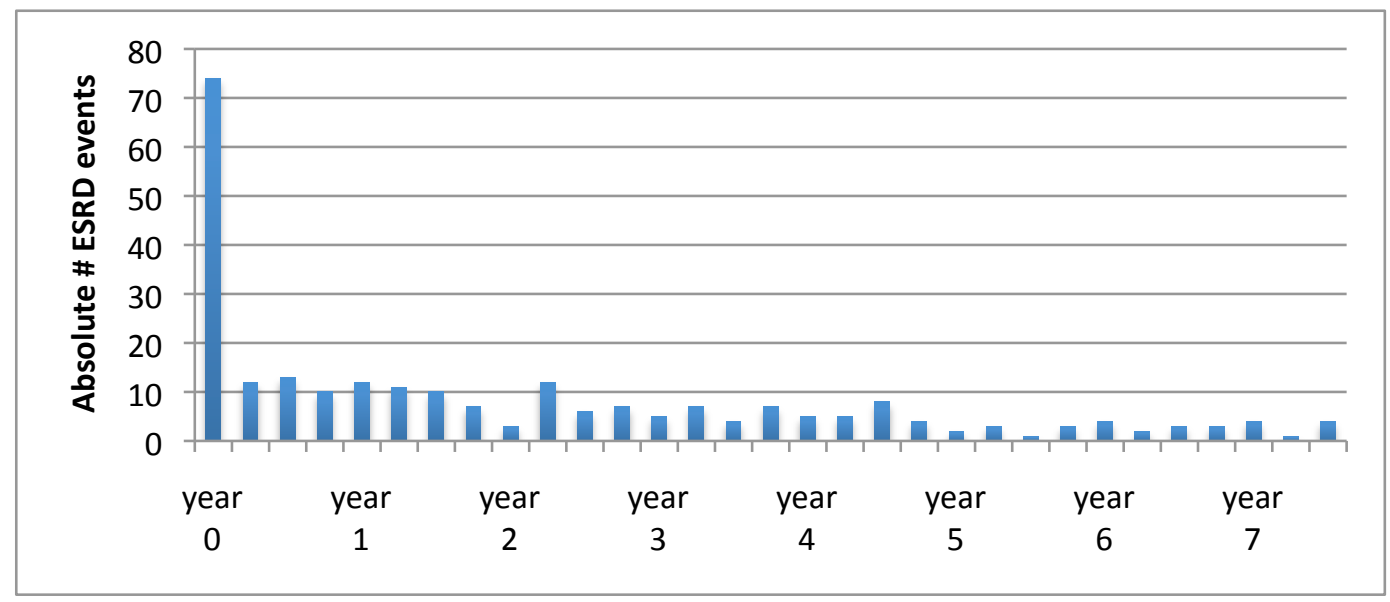

Capture rates for each of the individual comorbidities (HTN, DM, CKD, MI) were determined across the years to ensure consistency over time, across coding systems 
(ICD-9 and ICD-10) and with the prior published literature. This is demonstrated in Figure 4.3. The rate of the primary outcome (ESRD requiring RRT) was similarly plotted. Variability regarding outcome rates for PN was demonstrated in the earlier years of the procedure and stability was demonstrated in more recent years as the procedure became more widely accepted and performed (Figure 4.4).

Figure 4.3: Percentage of patients diagnosed with individual comorbidities based on year of diagnosis.

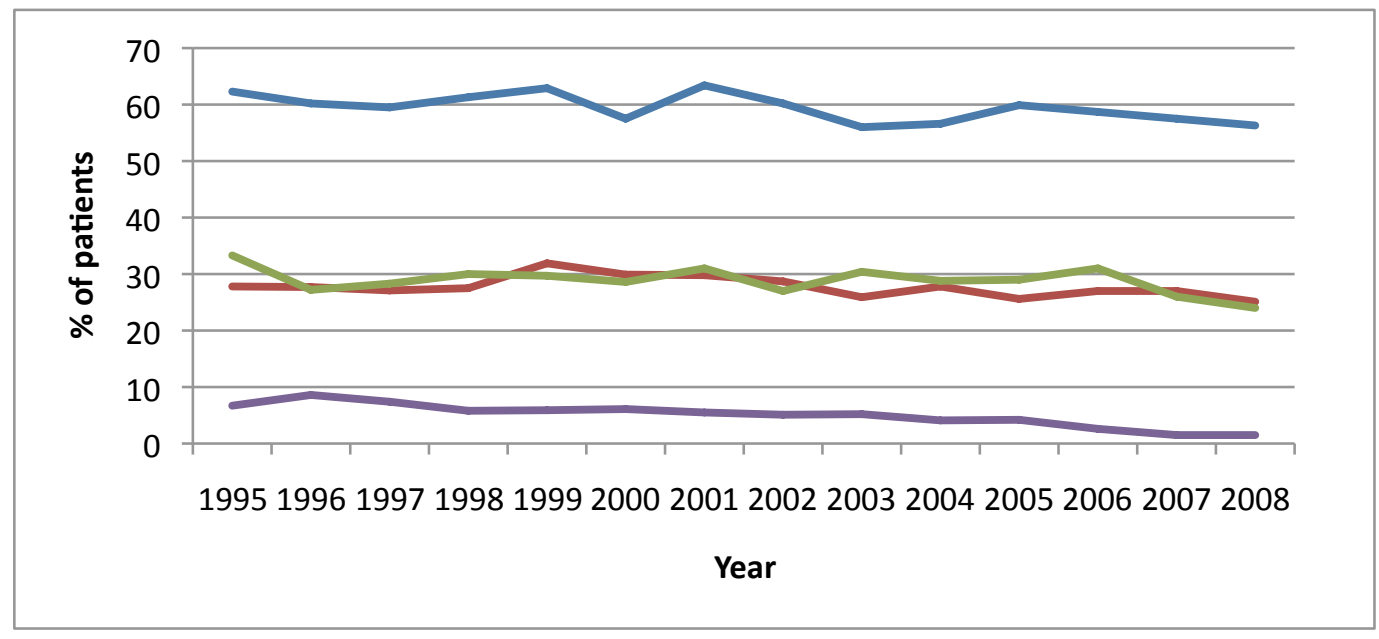


Figure 4.4: Rates of ESRD during subsequent post-operative years stratified by surgery ( $\mathrm{PN}$ vs. $\mathrm{RN}$ ) for patients diagnosed in various time periods.

a) Years 1995-1999

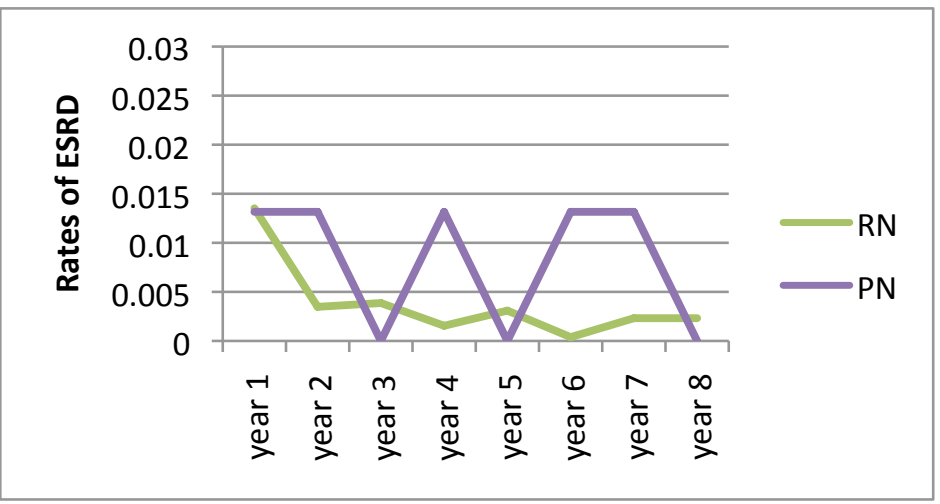

b) Years $2000-2004$

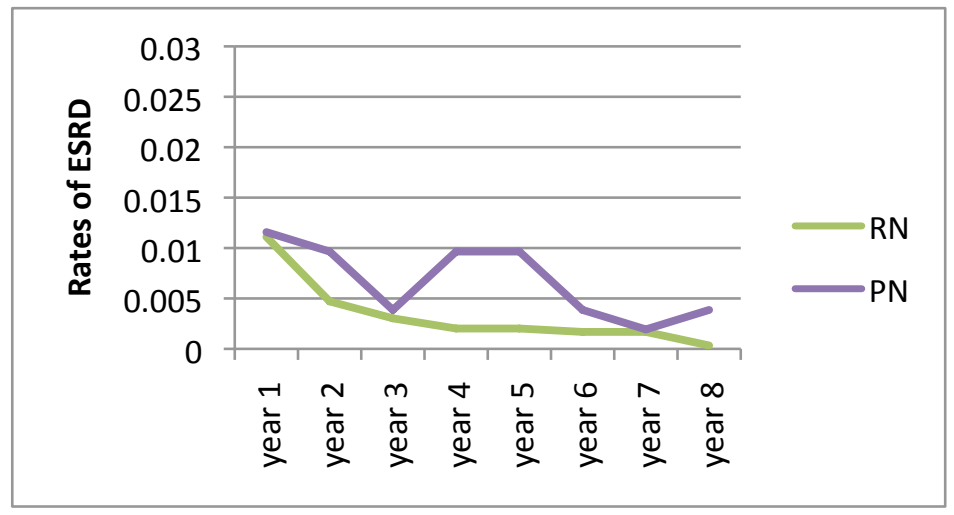

c) Years $2005-2010$

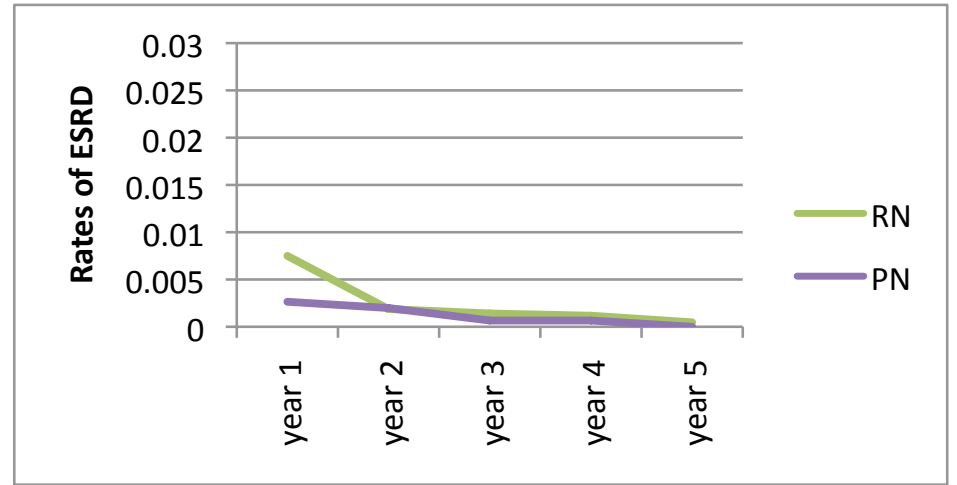




\subsubsection{Descriptive statistics - Outcomes}

The overall rate of patients who developed ESRD requiring RRT in the postoperative period was $2.5 \%$ (292 patients), with $2.2 \%$ (47 patients) amongst those undergoing PN compared to 2.5\% (245 patients) amongst those undergoing RN (Table 4.2). Among patients requiring RRT, 23 patients (7.8\%) underwent renal transplantation as their initial method of RRT.

Table 4.2: Overall rates of primary and secondary outcomes

\begin{tabular}{|c|l|l|l|}
\hline & $\begin{array}{l}\text { Radical } \\
\text { nephrectomy }\end{array}$ & $\begin{array}{l}\text { Partial } \\
\text { nephrectomy }\end{array}$ & Overall \\
\hline $\begin{array}{l}\text { Primary outcome } \\
\text { Renal replacement therapy }\end{array}$ & $245(2.5 \%)$ & $47(2.2 \%)$ & $292(2.5 \%)$ \\
$\begin{array}{l}\text { Secondary outcomes } \\
\text { Myocardial infarction }\end{array}$ & $\begin{array}{l}304(3.1 \%) \\
\text { New-onset CKD }\end{array}$ & $\begin{array}{l}31(1.5 \%) \\
1,838(18.7 \%)\end{array}$ & $\begin{array}{l}335(2.8 \%) \\
\text { Acute dialysis }\end{array}$ \\
\hline
\end{tabular}

Abbreviations: $\mathrm{CKD}=$ chronic kidney disease

Patients with greater pre-operative comorbidity developed ESRD requiring RRT at rates higher than those having less comorbidity. This trend was demonstrated across various measures of comorbidity, including Charlson comorbidity score (Figure 4.5) and weighted ADG score (Figure 4.6). Similarly, increased rates of RRT were identified amongst patients diagnosed with pre-operative hypertension, pre-operative DM, preoperative MI, and pre-operative CKD (Figure 4.7). Rates of ESRD requiring RRT increased across age groups (Figure 4.8). 
Figure 4.5: Rates of ESRD (\%) stratified by Charlson comorbidity score

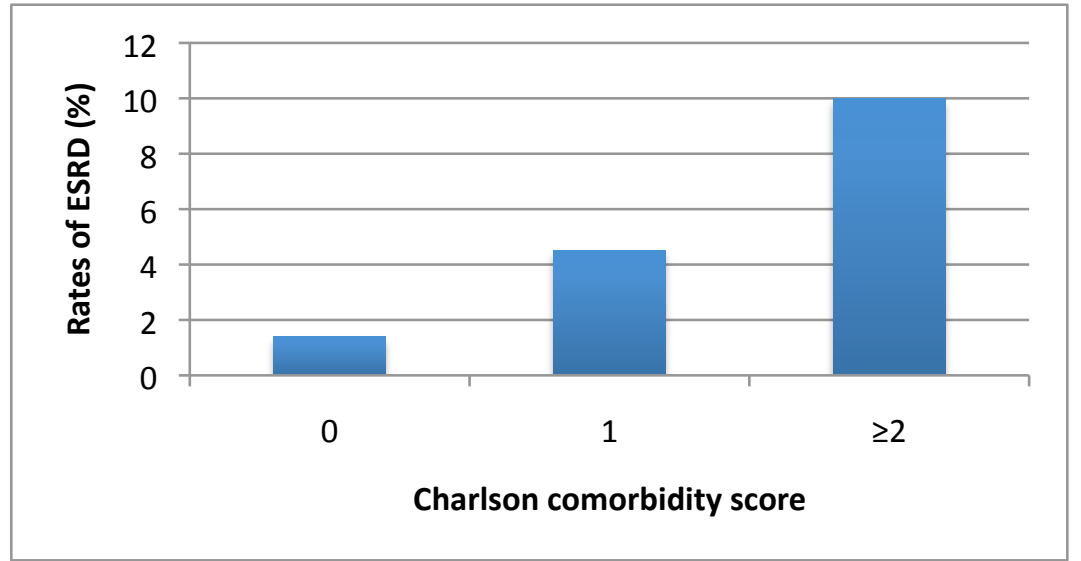

Figure 4.6: Rates of ESRD (\%) stratified by weighted ADG score (quintiles)

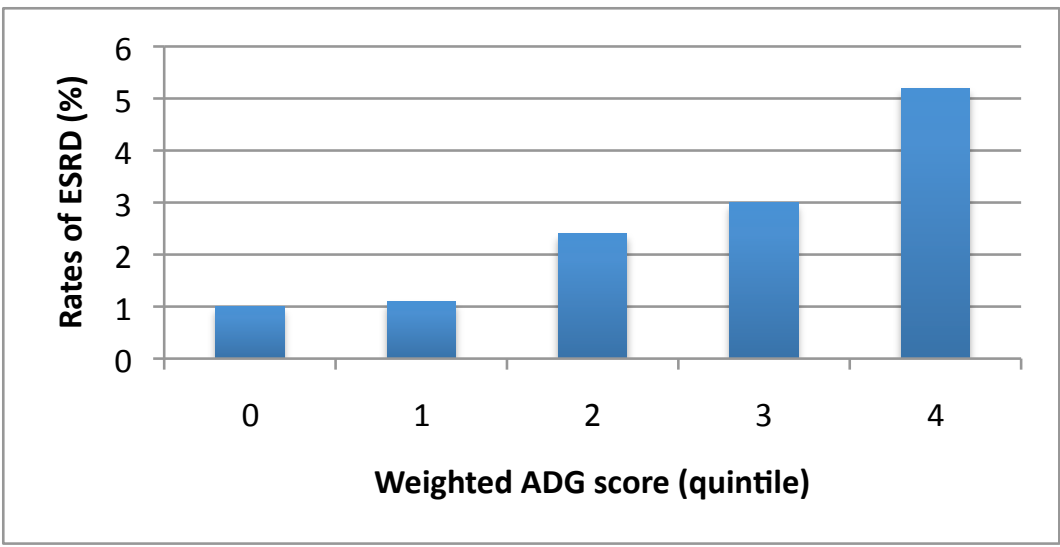

Figure 4.7: Rates of ESRD (\%) stratified by pre-operative comorbidity

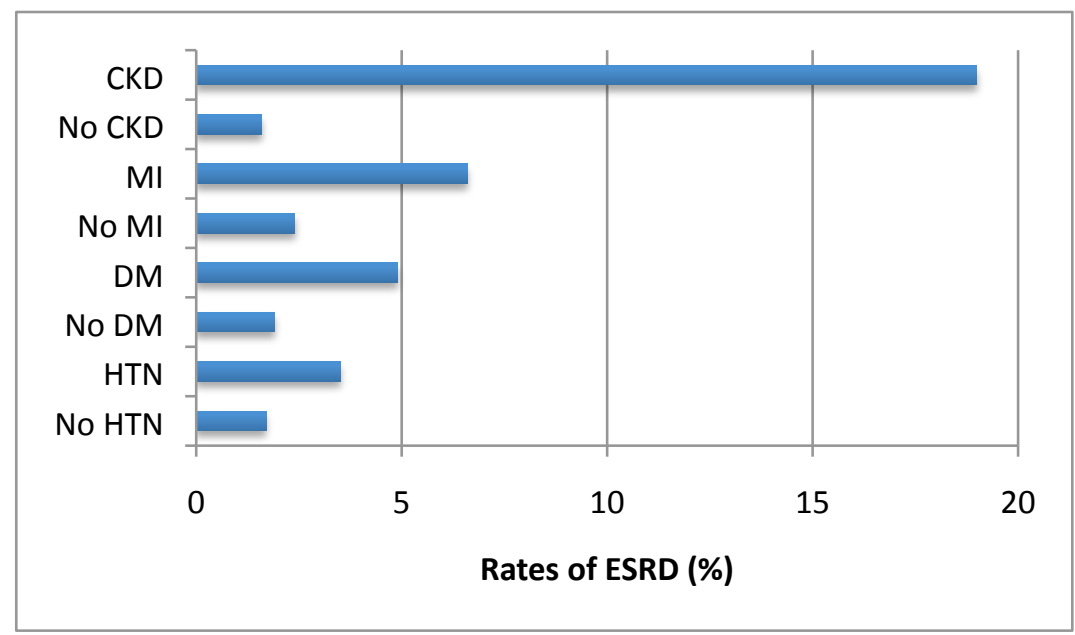


Figure 4.8: Rates of ESRD (\%) by stratified by age group

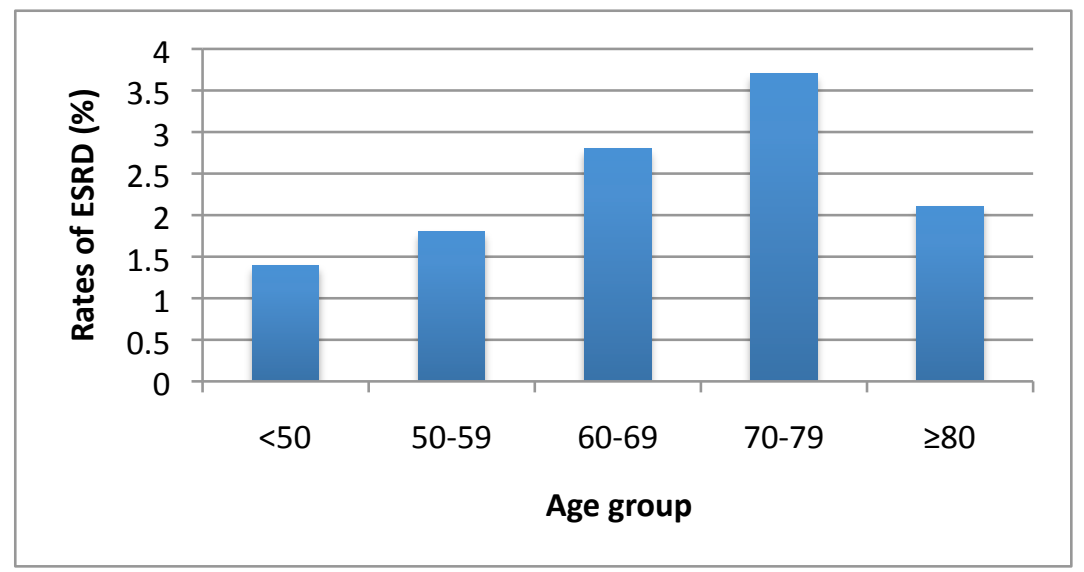

The overall (unadjusted) rates of patients requiring post-operative acute dialysis therapy, being hospitalized for post-operative MI, and developing new-onset CKD were $2.2 \%$ (311 patients), 2.8\% (335 patients), and 16.9\% (2,012 patients), respectively. The unadjusted rate of each adverse outcome was greater for those undergoing $\mathrm{RN}$ compared to those undergoing PN (Table 4.2).

\subsubsection{Development of propensity scores}

Propensity scores were developed using logistic regression analysis to assess the propensity of undergoing PN compared to RN. All variables believed to be clinically significant in contributing to a patient's likelihood of undergoing PN or RN were included in modeling the propensity score. Variables utilized in obtaining the propensity score were demographic variables (gender, age, year of diagnosis), tumour characteristics (histology), and comorbidities (HTN, DM, MI, CKD). Propensity scores ranged from 0.007 to 0.613 , and histograms demonstrating the range and distribution of scores are presented in Figure 4.9. 
Figure 4.9: Histograms demonstrating range and distribution of propensity scores

a) Overall cohort

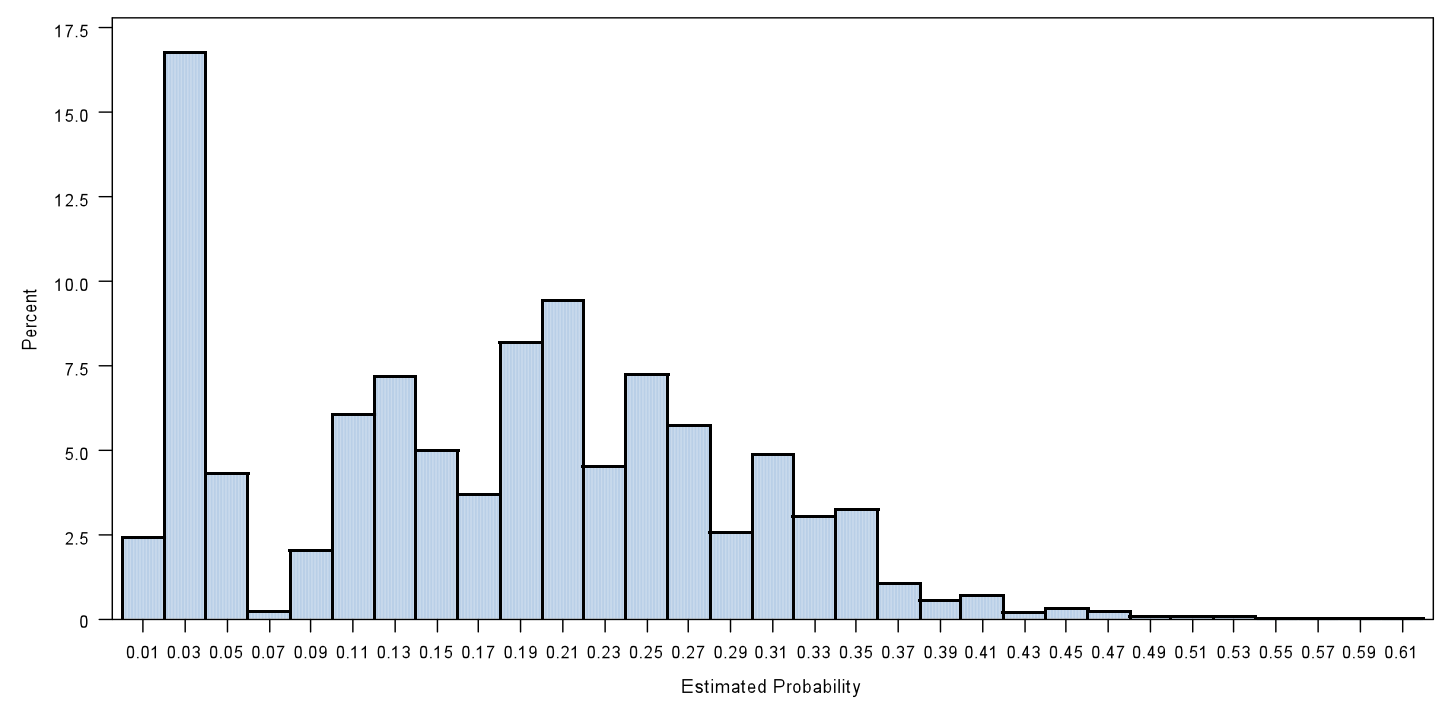

b) Patients undergoing RN

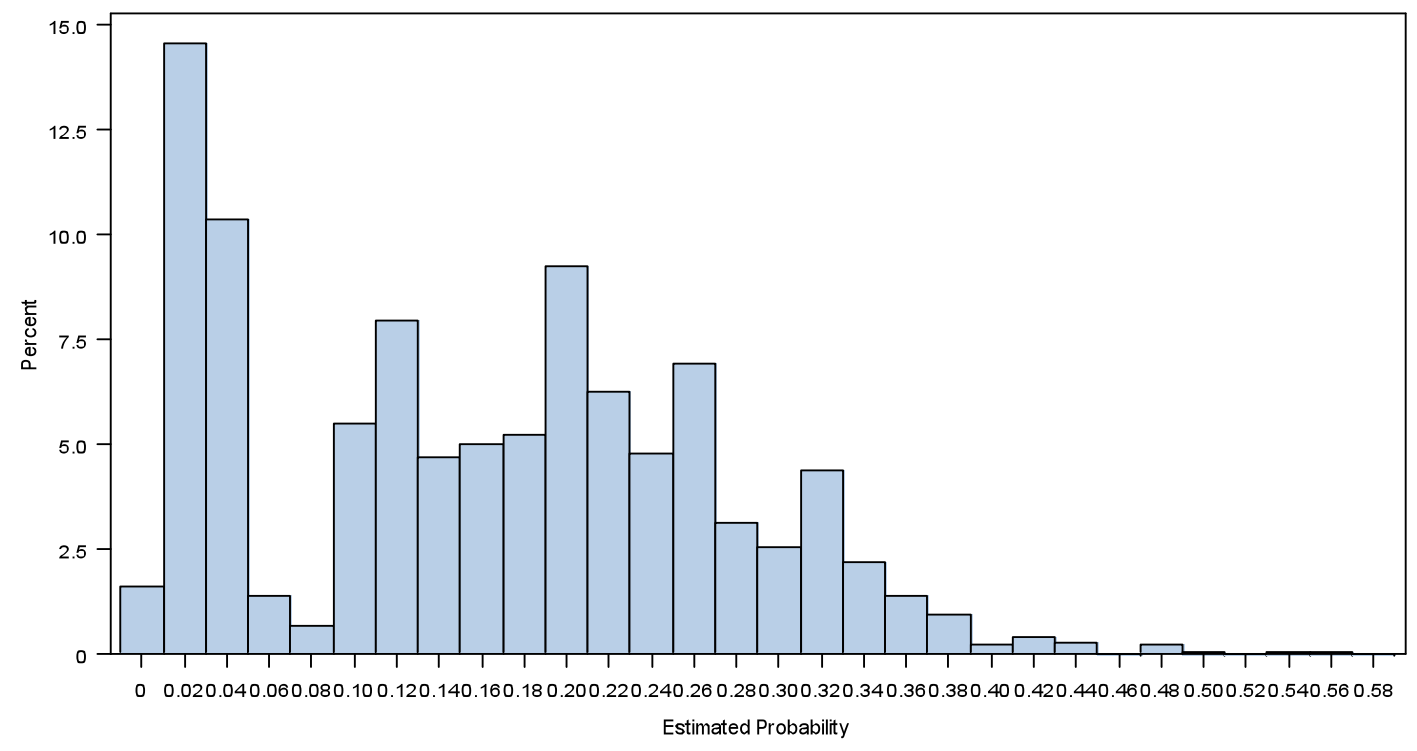


c) Patients undergoing PN

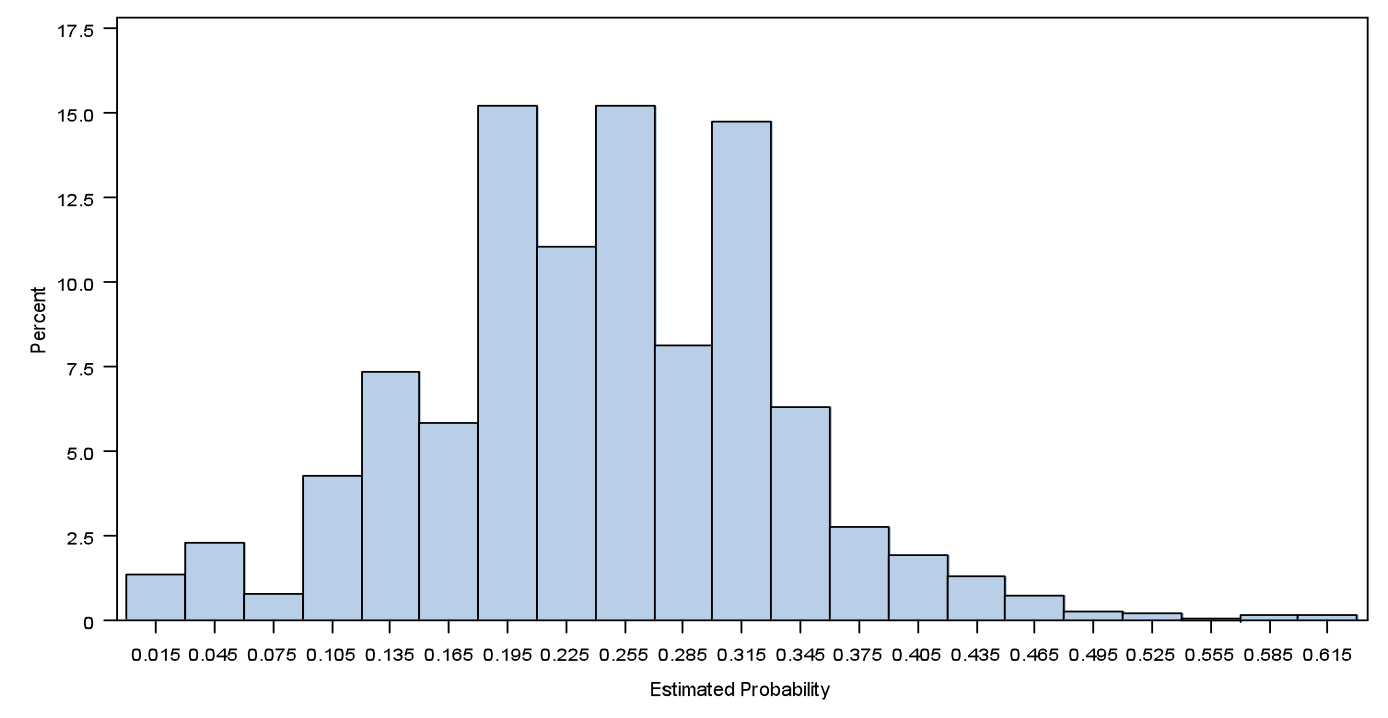

Propensity scores were also converted into a categorical variable (quintiles) for analysis. The distribution of demographic and clinical variables was assessed within each quintile. Improved balance between treatment groups with respect to the distribution of covariates was noted within each quintile compared to initial comparisons of the overall cohort (Appendix A, Table 1).

Propensity scores were then utilized to adjust for differences in treatment groups while assessing the impact of surgical treatment on the primary and secondary outcomes. Propensity scores were placed into the time-to-event models both as a continuous and as a categorical (quintile) variable (in separate models). No significant differences were identified between the two methods of utilizing propensity score regarding the relationship between surgical approach and the various outcomes. Propensity score as a continuous variable was utilized in the primary analysis. 


\subsection{Primary outcome - ESRD requiring RRT}

Kaplan-Meier curve (ESRD)

Kaplan Meier curves were generated to demonstrate risk of ESRD stratified by surgical treatment (Figure 4.10). A comparison of curves using the log-rank test did not demonstrate significant differences between the two surgical treatments groups $(\mathrm{p}=0.62)$.

Figure 4.10: Kaplan Meier curves stratified by surgical treatment for outcome of ESRD requiring RRT.

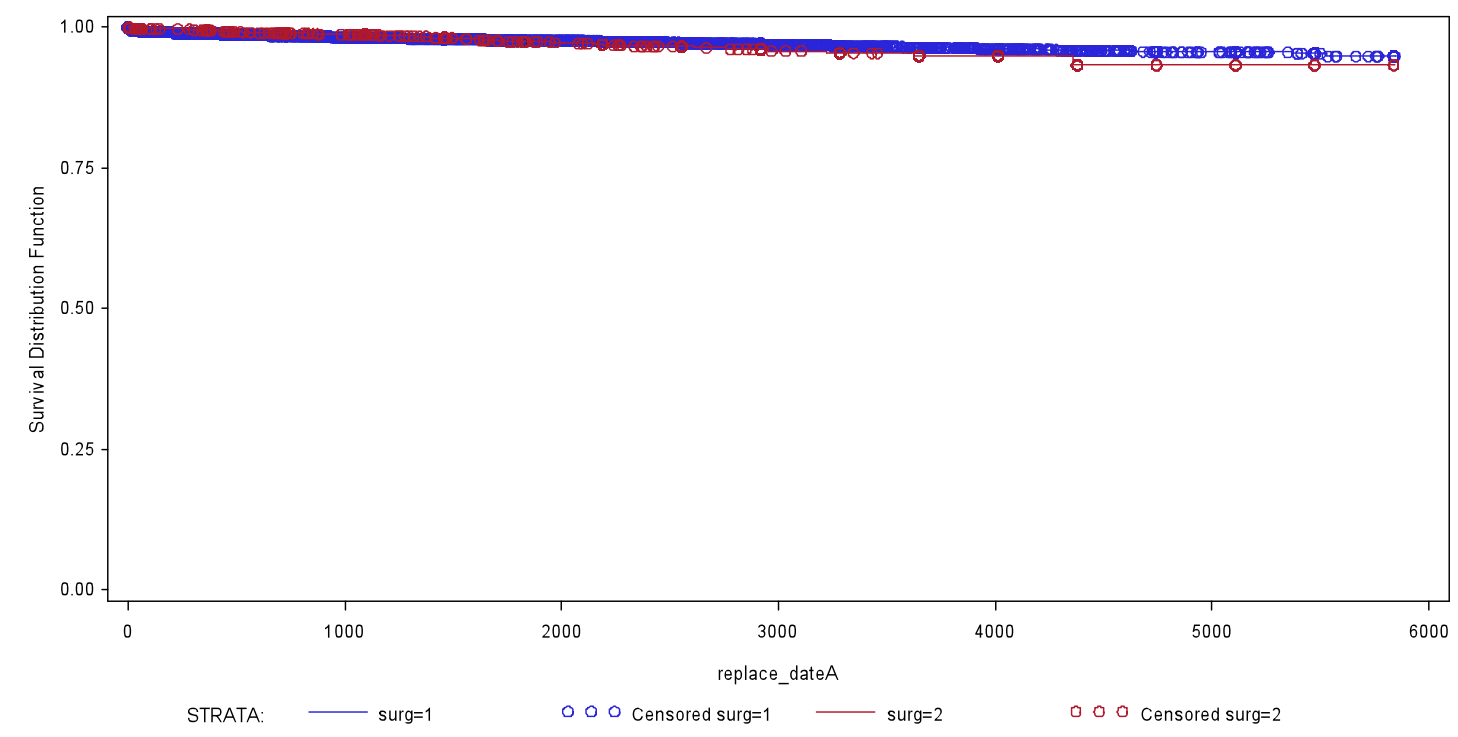

Cox proportional hazards model (ESRD)

No differences between surgical treatment groups were identified on univariable analysis using a Cox proportional hazards model (HR 1.08, CI 0.79-1.48). Variables significantly predictive of time to ESRD on univariable analysis included male gender 
(HR 1.23, 0.97-1.57), increasing patient age group $(\mathrm{p}<0.05)$, most recent diagnosis year group (HR 0.49, 0.35-0.68), unspecified histology (HR 1.85, 1.36-2.51), Charlson comorbidity score $=1($ HR 3.57, 2.75-4.63) Charlson comorbidity score $\geq 2$ (HR 9.16, 6.75-12.43), increasing weighted ADG score (HR 1.07 per unit, 1.06-1.08), pre-operative HTN (HR 2.12, 1.68-2.68), pre-operative DM (HR 2.81, 1.39-5.67), and pre-operative CKD (HR 14.44, 10.48-19.89). (Table 4.3)

Table 4.3: Univariable Cox proportional hazards model (ESRD)

\begin{tabular}{|l|l|l|}
\hline & Univariable & \\
\hline & HR (CI) & p-value \\
\hline Surgery & & \\
\hline RN & Ref & \\
\hline PN & $1.08(0.79-1.48)$ & 0.62 \\
\hline Sex & & \\
\hline Female & Ref & \\
\hline Male & $1.23(0.97-1.57)$ & 0.08 \\
\hline Age group & & $<0.05$ \\
\hline$<50$ & Ref & \\
\hline $50-59$ & $1.45(0.94-2.24)$ & 0.09 \\
\hline $60-69$ & $2.32(1.55-3.48)$ & $<0.05$ \\
\hline $70-79$ & $3.21(2.15-4.79)$ & $<0.05$ \\
\hline$\geq 80$ & $2.19(1.18-4.05)$ & $<0.05$ \\
\hline Year group & & $<0.05$ \\
\hline $1995-1999$ & Ref & \\
\hline $2000-2004$ & $0.89(0.68-1.16)$ & 0.41 \\
\hline $2005-2010$ & $0.49(0.35-0.68)$ & $<0.05$ \\
\hline Histology & & $<0.05$ \\
\hline Clear cell & Ref & \\
\hline Papillary & $1.17(0.61-2.24)$ & 0.64 \\
\hline Chromophobe & $0.61(0.22-1.69)$ & 0.34 \\
\hline NOS & $1.85(1.36-2.51)$ & $<0.05$ \\
\hline Charlson score & & $<0.05$ \\
\hline 0 & Ref & \\
\hline 1 & $3.57(2.75-4.63)$ & $<0.05$ \\
\hline$\geq 2$ & $9.16(6.75-12.43)$ & $<0.05$ \\
\hline ADG (weighted) & & \\
\hline & $1.07(1.06-1.08)$ & $<0.05$ \\
\hline HTN & & \\
\hline No & Ref & \\
\hline Yes & $2.12(1.68-2.68)$ & $<0.05$ \\
\hline MI & & \\
\hline No & Ref & \\
\hline
\end{tabular}




\begin{tabular}{|c|l|l|}
\hline Yes & $2.81(1.39-5.67)$ & $<0.05$ \\
\hline DM & & \\
\hline No & Ref & \\
\hline Yes & $3.11(2.45-3.95)$ & $<0.05$ \\
\hline CKD & & \\
\hline No & Ref & \\
\hline Yes & $14.44(10.48-19.89)$ & $<0.05$ \\
\hline
\end{tabular}

Abbreviations: $\mathrm{HR}=$ hazards ratio $\mathrm{CI}=$ confidence interval; $\mathrm{RN}=$ radical nephrectomy; $\mathrm{PN}=$ partial nephrectomy; NOS = not otherwise specified; ADG = Johns Hopkins' Aggregated Diagnosis Groups; HTN = hypertension; $\mathrm{MI}=$ myocardial infarction; DM = diabetes mellitus; $\mathrm{CKD}=$ chronic kidney disease.

Various multivariable Cox proportional hazards models were assessed utilizing distinct methods available to control for patient comorbidity - Charlson comorbidity score, ADG score (weighted), and individually captured comorbid diagnoses (Table 4.4). Models utilizing Charlson comorbidity score and ADG (weighted) score are demonstrated in the appendix (Appendix A, Tables 2 and 3). Overall findings were similar between the various models. Surgical approach was not significantly associated with ESRD requiring RRT in any of the models. Variables associated with a significantly increased hazard of ESRD requiring RRT across all the models included patient age group (60-69, 70-79) and unspecified histology. Each measure of increasing patient comorbidity was associated with increased hazards of developing ESRD, including Charlson comorbidity score, weighted ADG score, pre-operative HTN, pre-operative DM, and pre-operative CKD. 
Table 4.4: Univariable and multivariable Cox proportional hazards model with individual comorbidities (ESRD)

\begin{tabular}{|l|l|l|l|l|}
\hline & Univariable & & Multivariable & \\
\hline & HR (CI) & p-value & HR (CI) & p-value \\
\hline Surgery & & & & \\
\hline RN & Ref & & Ref & \\
\hline PN & $0.63(0.79-1.48)$ & 0.62 & $0.75(0.54-1.05)$ & 0.09 \\
\hline Sex & & & & \\
\hline Female & Ref & & Ref & \\
\hline Male & $1.23(0.97-1.57)$ & 0.08 & $1.08(0.84-1.37)$ & 0.55 \\
\hline Age group & & $<0.05$ & & $<0.05$ \\
\hline$<50$ & Ref & & Ref & \\
\hline $50-59$ & $1.45(0.94-2.24)$ & 0.09 & $1.21(0.77-1.87)$ & 0.39 \\
\hline $60-69$ & $2.32(1.55-3.48)$ & $<0.05$ & $1.65(1.09-2.49)$ & $<0.05$ \\
\hline $70-79$ & $3.21(2.15-4.79)$ & $<0.05$ & $1.93(1.27-2.91)$ & $<0.05$ \\
\hline$\geq 80$ & $2.19(1.18-4.05)$ & $<0.05$ & $1.17(0.63-1.12)$ & 0.61 \\
\hline Year group & & $<0.05$ & & $<0.05$ \\
\hline $1995-1999$ & Ref & & Ref & \\
\hline $2000-2004$ & $0.89(0.68-1.16)$ & 0.41 & $0.85(0.64-1.12)$ & 0.25 \\
\hline 2005-2010 & $0.49(0.35-0.68)$ & $<0.05$ & $0.37(0.26-0.54)$ & $<0.05$ \\
\hline Histology & & $<0.05$ & & $<0.05$ \\
\hline Clear cell & Ref & & Ref & \\
\hline Papillary & $1.17(0.61-2.24)$ & 0.64 & $0.92(0.47-1.77)$ & 0.81 \\
\hline Chromophobe & $0.61(0.22-1.69)$ & 0.34 & $0.63(0.22-1.75)$ & 0.37 \\
\hline NOS & $1.85(1.36-2.51)$ & $<0.05$ & $1.64(1.19-2.26)$ & $<0.05$ \\
\hline HTN & & & & \\
\hline No & Ref & & Ref & \\
\hline Yes & $2.12(1.68-2.68)$ & $<0.05$ & $1.52(1.19-1.94)$ & $<0.05$ \\
\hline MI & & & & \\
\hline No & Ref & & Ref & \\
\hline Yes & $2.81(1.39-5.67)$ & $<0.05$ & $1.24(0.60-2.55)$ & 0.55 \\
\hline DM & & & & \\
\hline No & Ref & & Ref & \\
\hline Yes & $3.11(2.45-3.95)$ & $<0.05$ & $2.07(1.61-2.65)$ & $<0.05$ \\
\hline CKD & $14.44(10.48-19.89)$ & $<0.05$ & $16.81(12.91-21.89)$ & $<0.05$ \\
\hline No & & & & \\
\hline Yes & & & Ref & \\
\hline Abbrvian & & & \\
\hline
\end{tabular}

Abbreviations: $\mathrm{HR}=$ hazards ratio; $\mathrm{CI}=$ confidence interval; $\mathrm{RN}=$ radical nephrectomy; $\mathrm{PN}=$ partial nephrectomy; NOS = not otherwise specified; $\mathrm{HTN}=$ hypertension; $\mathrm{MI}=$ myocardial infarction; $\mathrm{DM}=$ diabetes mellitus; $\mathrm{CKD}=$ chronic kidney disease. 


\section{Propensity score model (ESRD)}

No significant relationship was identified between surgical treatment and the development of ESRD requiring RRT (HR 1.04, 95\% CI 0.75-1.45) while utilizing a continuous propensity score variable to control for confounding factors. Similar results were demonstrated utilizing propensity score as a categorical variable (quintiles) (Appendix A, Table 4).

Competing risks analysis (ESRD)

Using the Pepe-Mori test to compare the cumulative incidence functions, no significant difference was identified between PN and RN in their relationship to the development of ESRD requiring RRT while controlling for the outcome of overall mortality $(\mathrm{p}=0.37)$. (Figure 4.11)

Figure 4.11: Competing risks analysis: cumulative probability curves stratified by surgical treatment for the outcome of ESRD requiring RRT.

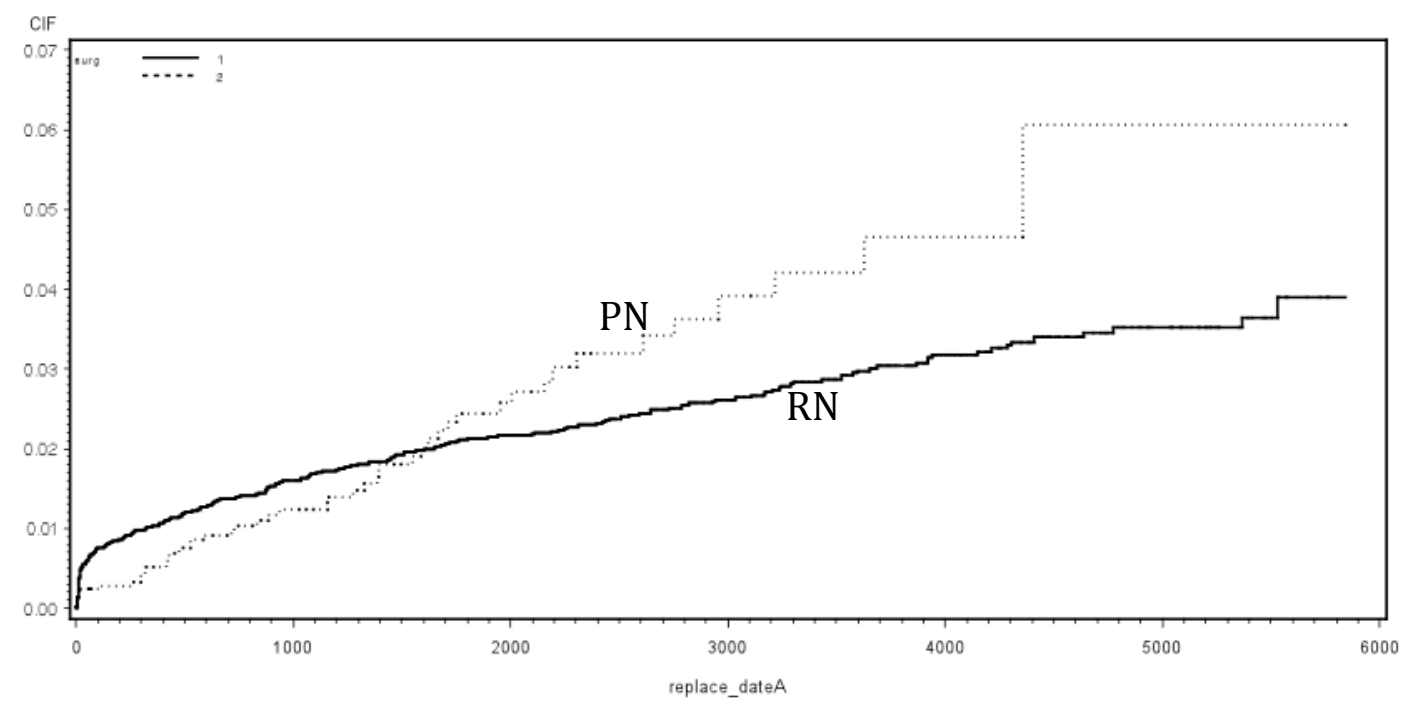


Summary (ESRD)

Significant differences were not identified between RN and PN regarding the outcome of ESRD. A comparison of curves using the log-rank test did not demonstrate significant differences between the two surgical treatments groups $(\mathrm{p}=0.62)$. No significant differences were identified on either the multivariable Cox PH models adjusting for individual comorbidities (HR 0.75, 95\% CI 0.54-1.05) or propensity score (HR 1.04, 95\% CI 0.75-1.45). Furthermore, no significant differences were identified between groups when accounting for the competing risk of overall survival $(\mathrm{p}=0.37)$.

\subsection{Primary outcome - ESRD requiring RRT (Modern cohort, 2003-2010) Baseline characteristics}

Baseline characteristics of the modern cohort are demonstrated in Appendix A, Table 5. A total of 7,230 individuals were analyzed in this cohort with 1,746 patients (24\%) having undergone PN compared to 5,484 (76\%) patients having undergone RN. Median follow-up was 41 months for the overall cohort. Rates of major comorbidity are similar between the groups across both composite comorbidity scores (Charlson Comorbidity score, $\mathrm{ADG}$ ) and individually identified risk factors (HTN, DM, prior CKD, prior MI). (Appendix A, Table 5)

Kaplan-Meier curve - ESRD (Modern cohort)

Kaplan Meier curves were created to demonstrate the risk of ESRD stratified by surgical treatment within a modern cohort (2003-2010) (Figure 4.12). A comparison of curves using the log-rank test demonstrated significant benefit for PN in preventing ESRD requiring RRT $(\mathrm{p}<0.05)$. 
Figure 4.12: Kaplan Meier curves stratified by surgical treatment for outcome of ESRD requiring RRT (modern cohort, 2003-2010).

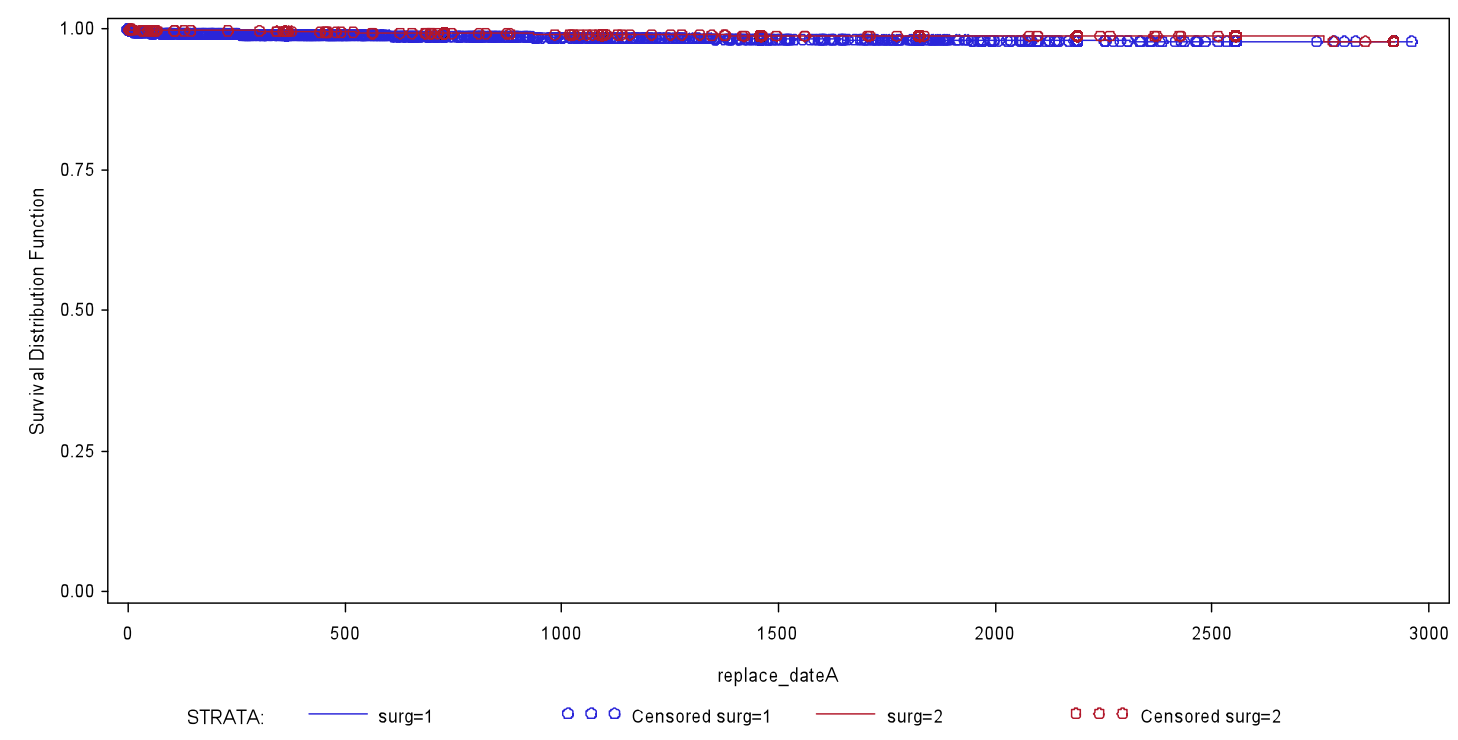

Cox proportional hazards model - ESRD (Modern cohort)

Using a multivariable Cox proportional hazards model to assess this cohort, PN was significantly associated with a decreased likelihood of ESRD requiring RRT (HR $0.43,95 \%$ CI $0.25-0.75)$. Other variables associated with significantly increased hazards of ESRD requiring RRT were pre-operative diagnoses of DM (HR 1.77, 95\% CI 1.172.67) or CKD (HR 20.26, 95\% CI 13.30-30.86). (Table 4.5) A sensitivity analysis utilizing sequential years to define the modern cohort is presented in table 4.5. The table demonstrates consistent findings, with PN imparting an increasing benefit compared to $\mathrm{RN}$ in the prevention of ESRD as an increasingly modern cohort is utilized. 
Table 4.5: Univariable and multivariable Cox proportional hazards model with individual comorbidities in the modern cohort - 2003-2010 (ESRD)

\begin{tabular}{|c|c|c|c|c|}
\hline & Univariable & & Multivariable & \\
\hline & $\mathrm{HR}(\mathrm{CI})$ & p-value & $\mathrm{HR}(\mathrm{CI})$ & p-value \\
\hline \multicolumn{5}{|l|}{ Surgery } \\
\hline $\mathrm{RN}$ & Ref & & Ref & \\
\hline $\mathrm{PN}$ & $0.59(0.35-1.01)$ & 0.06 & $0.44(0.25-0.75)$ & $<0.05$ \\
\hline \multicolumn{5}{|l|}{ Sex } \\
\hline Female & Ref & & Ref & \\
\hline Male & $1.29(0.85-1.95)$ & 0.22 & $1.07(0.70-1.63)$ & 0.75 \\
\hline Age group & & $<0.05$ & & 0.53 \\
\hline$<50$ & Ref & & Ref & \\
\hline $50-59$ & $0.86(0.41-1.82)$ & 0.70 & $0.74(0.35-1.57)$ & 0.44 \\
\hline $60-69$ & $1.75(0.92-3.36)$ & 0.08 & $1.11(0.57-2.17)$ & 0.75 \\
\hline $70-79$ & $2.74(1.45-5.17)$ & $<0.05$ & $1.20(0.61-2.33)$ & 0.58 \\
\hline$\geq 80$ & $1.87(0.75-4.70)$ & 0.17 & $0.77(0.30-1.99)$ & 0.59 \\
\hline Histology & & 0.05 & & 0.09 \\
\hline Clear cell & Ref & & Ref & \\
\hline Papillary & $1.37(0.67-2.79)$ & 0.38 & $1.18(0.57-2.42)$ & 0.65 \\
\hline Chromophobe & $0.39(0.09-1.62)$ & 0.19 & $0.43(0.10-1.80)$ & 0.25 \\
\hline NOS & $1.60(1.04-2.45)$ & 0.03 & $1.55(1.00-2.40)$ & 0.05 \\
\hline Year & $0.86(0.78-0.95)$ & $<0.05$ & $0.85(0.76-0.94)$ & $<0.05$ \\
\hline \multicolumn{5}{|l|}{ HTN } \\
\hline No & Ref & & Ref & \\
\hline Yes & $2.28(1.52-3.42)$ & $<0.05$ & $1.52(0.99-2.32)$ & 0.05 \\
\hline \multicolumn{5}{|l|}{ MI } \\
\hline No & Ref & & Ref & \\
\hline Yes & $4.55(1.67-12.38)$ & $<0.05$ & $1.91(0.68-5.32)$ & 0.21 \\
\hline \multicolumn{5}{|l|}{$\mathrm{DM}$} \\
\hline No & Ref & & Ref & \\
\hline Yes & $2.93(1.98-4.35)$ & $<0.05$ & $1.77(1.17-2.67)$ & $<0.05$ \\
\hline \multicolumn{5}{|l|}{ CKD } \\
\hline No & Ref & & Ref & \\
\hline Yes & $22.83(15.42-33.82)$ & $<0.05$ & $20.26(13.30-30.86)$ & $<0.05$ \\
\hline
\end{tabular}

Abbreviations: $\mathrm{HR}=$ hazards ratio; $\mathrm{CI}=$ confidence interval; $\mathrm{RN}=$ radical nephrectomy; $\mathrm{PN}=$ partial nephrectomy; NOS = not otherwise specified; $\mathrm{HTN}=$ hypertension; $\mathrm{MI}=$ myocardial infarction; $\mathrm{DM}=$ diabetes mellitus; $\mathrm{CKD}=$ chronic kidney disease . 
Table 4.6: Analysis of the impact of PN on outcome of ESRD analyzing sequentially by year to define the modern cohort

\begin{tabular}{|l|l|l|}
\hline Year of analysis & HR (CI) & p-value \\
\hline$\geq 1995$ & $0.77(0.55-1.07)$ & 0.12 \\
\hline$\geq 1996$ & $0.83(0.59-1.17)$ & 0.31 \\
\hline$\geq 1997$ & $0.85(0.60-1.19)$ & 0.35 \\
\hline$\geq 1998$ & $0.85(0.60-1.20)$ & 0.37 \\
\hline$\geq 1999$ & $0.88(0.63-1.25)$ & 0.50 \\
\hline$\geq 2000$ & $0.79(0.55-1.14)$ & 0.22 \\
\hline$\geq 2001$ & $0.65(0.43-1.00)$ & 0.05 \\
\hline$\geq 2002$ & $0.65(0.42-1.01)$ & 0.06 \\
\hline$\geq 2003$ & $0.44(0.25-0.75)$ & $<0.05$ \\
\hline$\geq 2004$ & $0.41(0.21-0.76)$ & $<0.05$ \\
\hline$\geq 2005$ & $0.37(0.18-0.76)$ & $<0.05$ \\
\hline$\geq 2006$ & $0.37(0.17-0.81)$ & $<0.05$ \\
\hline$\geq 2007$ & $0.28(0.10-0.73)$ & $<0.05$ \\
\hline$\geq 2008$ & $0.26(0.07-0.89)$ & $<0.05$ \\
\hline$\geq 2009$ & $0.11(0.01-0.88)$ & $<0.05$ \\
\hline & &
\end{tabular}

\section{Propensity score model - ESRD (Modern cohort)}

PN was associated with a decreased time to progression to ESRD compared to $\mathrm{RN}$ (HR $0.47,95 \%$ CI $0.27-0.82$ ) utilizing a propensity score as a continuous variable to control for confounding factors. Similar results were demonstrated utilizing propensity score as a categorical variable (quintiles) (Appendix A, Table 4).

Competing risks analysis - ESRD (Modern cohort)

Using the Pepe-Mori test to compare cumulative incidence functions, PN compared to RN was associated with a decreased time to the development of ESRD requiring RRT while controlling for the outcome of overall mortality $(\mathrm{p}<0.05)$ (Figure 4.13). 
Figure 4.13: Competing risks analysis: cumulative probability curves stratified by surgical treatment for the outcome of ESRD requiring RRT in the modern cohort (20032010).

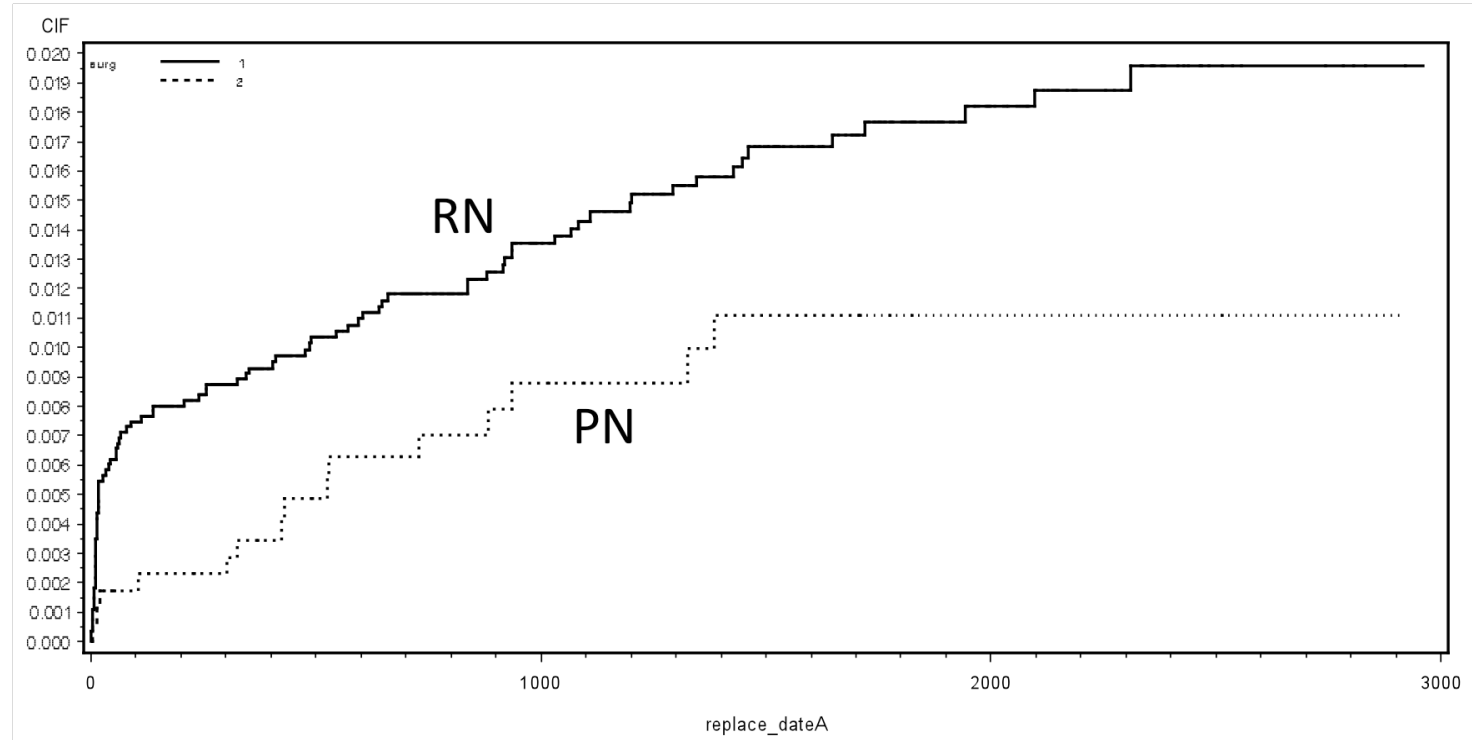

Summary (ESRD - modern cohort)

Significant differences were identified between $\mathrm{RN}$ and $\mathrm{PN}$ regarding the outcome of ESRD when analyzing the modern cohort. A comparison of curves using the log-rank test demonstrated significant differences between the two surgical treatments groups $(p<0.05)$. Significant differences were identified with a similar HR on both the multivariable Cox PH models adjusting for individual comorbidities (HR 0.43, 95\% CI 0.25-0.75) and propensity score (HR 0.47, 95\% CI 0.27-0.82). Differences persisted between groups when accounting for the competing risk of overall survival $(\mathrm{p}<0.05)$.

\subsection{Secondary outcome - Myocardial infarction}

Kaplan-Meier curve-Myocardial infarction 
Kaplan Meier curves were created to demonstrate the risk of hospitalization for MI stratified by surgical treatment (Figure 4.14). A comparison of curves with the logrank test demonstrated a benefit for PN in reducing the likelihood of $\mathrm{MI}(\mathrm{p}<0.05)$.

Figure 4.14: Kaplan Meier curves stratified by surgical treatment for outcome of MI.

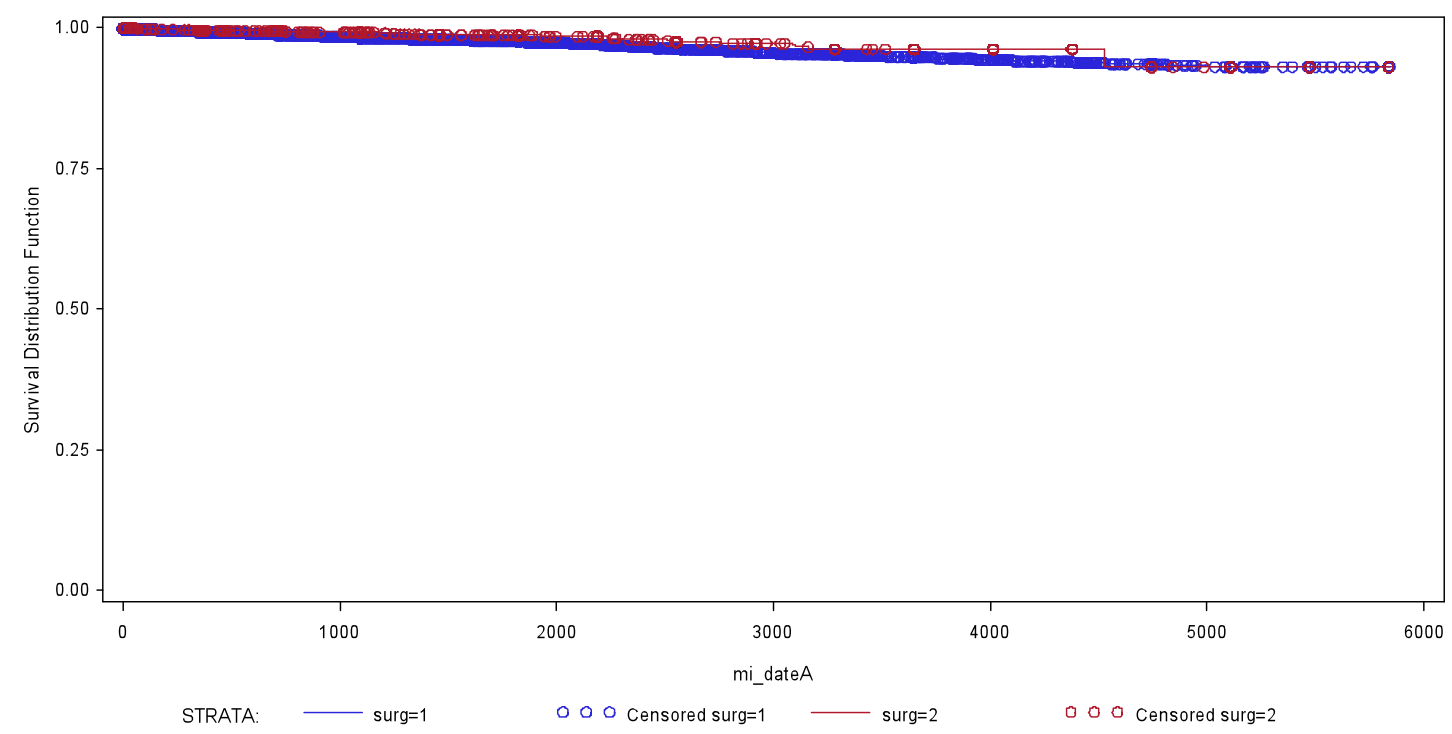

Cox proportional hazards model-Myocardial infarction

Univariable Cox proportional hazards analysis demonstrated that PN was associated with a decreased hazard of subsequent MI (HR 0.62, 95\% CI 0.43-0.90). Other variables significant on univariable analysis included male gender (HR 1.46, 95\% CI 1.16-1.84), increasing age group $(\mathrm{p}<0.05)$, recent diagnosis year group (HR $0.38,95 \%$ CI 0.27-0.54), unspecified histology (HR 1.44, 95\% CI 1.09-1.89), pre-operative HTN (HR 
$1.45,95 \%$ CI 1.17-1.80), pre-operative DM (HR 2.19, 95\% CI 1.73-2.78), and preoperative CKD (HR 2.19, 95\% CI 1.47-3.28).

On multivariable analysis, however, surgical treatment was no longer a significant predictor of time to MI (HR $0.76,95 \%$ CI $0.52-1.12)$. Variables that remained significant on multivariable analysis included male gender (HR 1.52, 95\% CI 1.20-1.91), increasing age group (HR 5.58, 95\% CI 3.31-9.44), recent diagnosis year group (HR 0.37, 95\% CI 0.25-0.54), pre-operative DM (HR 1.95, 95\% CI 1.54-2.49), and pre-operative CKD (HR $1.87,95 \%$ CI 1.24-2.84) (Table 4.7). 
Table 4.7: Univariable and multivariable Cox proportional hazards model (MI)

\begin{tabular}{|l|l|l|l|l|}
\hline & Univariable & & Multivariable & \\
\hline & HR (CI) & p-value & HR (CI) & p-value \\
\hline Surgery & & & & \\
\hline RN & Ref & & Ref & \\
\hline PN & $0.62(0.43-0.90)$ & $<0.05$ & $0.76(0.52-1.12)$ & 0.17 \\
\hline Sex & & & & \\
\hline Female & Ref & & Ref & \\
\hline Male & $1.46(1.16-1.84)$ & $<0.05$ & $1.52(1.20-1.91)$ & $<0.05$ \\
\hline Age group & & $<0.05$ & & $<0.05$ \\
\hline$<50$ & Ref & & Ref & \\
\hline $50-59$ & $1.92(1.22-3.00)$ & $<0.05$ & $1.76(1.12-2.77)$ & $<0.05$ \\
\hline $60-69$ & $3.14(2.07-4.78)$ & $<0.05$ & $2.71(1.77-4.15)$ & $<0.05$ \\
\hline $70-79$ & $4.60(3.04-6.96)$ & $<0.05$ & $3.95(2.59-6.04)$ & $<0.05$ \\
\hline$\geq 80$ & $6.28(3.75-10.49)$ & $<0.05$ & $5.58(3.31-9.44)$ & $<0.05$ \\
\hline Year group & & $<0.05$ & & $<0.05$ \\
\hline $1995-1999$ & Ref & & Ref & \\
\hline $2000-2004$ & $0.78(0.61-1.00)$ & 0.05 & $0.80(0.62-1.04)$ & 0.10 \\
\hline $2005-2010$ & $0.38(0.27-0.54)$ & $<0.05$ & $0.37(0.25-0.54)$ & $<0.05$ \\
\hline Histology & & $<0.05$ & & 0.30 \\
\hline Clear cell & Ref & & & \\
\hline Papillary & $1.50(0.88-2.57)$ & 0.13 & & \\
\hline Chromophobe & $0.63(0.25-1.57)$ & 0.32 & & \\
\hline NOS & $1.44(1.09-1.89)$ & $<0.05$ & & \\
\hline HTN & & & & \\
\hline No & Ref & & Ref & \\
\hline Yes & $1.45(1.17-1.80)$ & $<0.05$ & $1.14(0.91-1.42)$ & 0.22 \\
\hline DM & Ref & & & \\
\hline No & $2.19(1.73-2.78)$ & $<0.05$ & $1.95(1.54-2.49)$ & $<0.05$ \\
\hline Yes & & & & \\
\hline CKD & Ref & & Ref & \\
\hline No & $2.19(1.47-3.28)$ & $<0.05$ & $1.87(1.24-2.84)$ & $<0.05$ \\
\hline Yes & & & \\
\hline Abbrev & & \\
\hline
\end{tabular}

Abbreviations: $\mathrm{HR}=$ hazards ratio; $\mathrm{CI}=$ confidence interval; $\mathrm{RN}=$ radical nephrectomy; $\mathrm{PN}=$ partial nephrectomy; $\mathrm{NOS}=$ not otherwise specified; $\mathrm{HTN}=$ hypertension; $\mathrm{DM}=$ diabetes mellitus; $\mathrm{CKD}=$ chronic kidney disease. 


\section{Propensity score model - Myocardial infarction}

No significant relationship was identified between surgical treatment and subsequent hospitalization for MI (HR 0.81, 95\% CI 0.55-1.19) while utilizing a continuous propensity score variable to control for confounding factors. Similar results were demonstrated utilizing propensity score as a categorical variable (quintiles) (Appendix A, Table 4).

Competing risks analysis - Myocardial infarction

PN was associated with a significantly decreased risk of hospitalization for MI compared to RN after comparing cumulative incidence functions while controlling for the outcome of all cause mortality $(\mathrm{p}<0.05)$. (Figure 4.15 )

Figure 4.15: Competing risks analysis: cumulative probability curves stratified by surgical treatment for the outcome of hospitalization for MI.

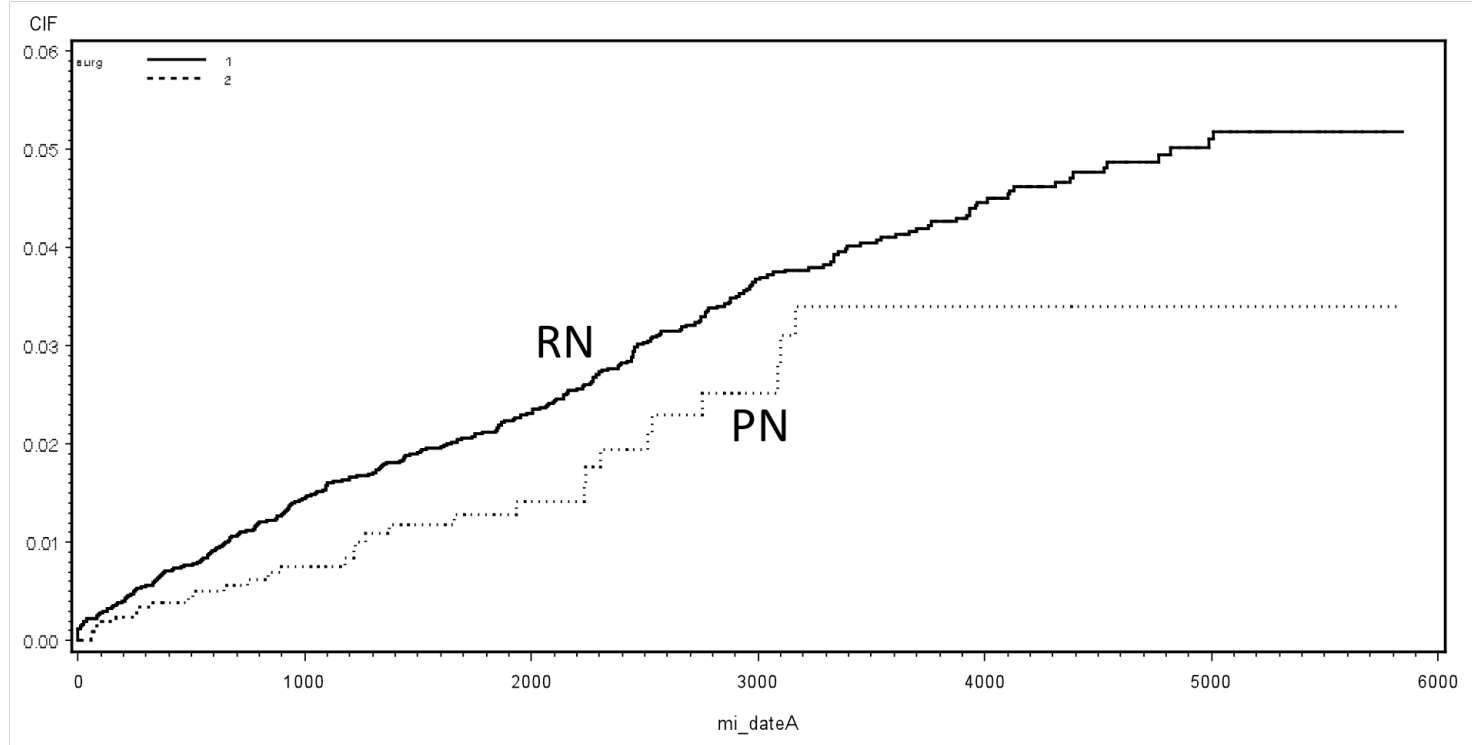


Summary (MI)

Results varied between surgical treatment groups (RN vs. PN) regarding the outcome of MI. A comparison of curves using the log-rank test demonstrated significant differences between the two surgical treatments groups $(\mathrm{p}<0.05)$. No significant differences were identified on either the multivariable Cox PH models adjusted for individual comorbidities (HR 0.76, 95\% CI 0.52-1.12) or propensity scores (HR 0.81, 95\% CI 0.551.19). The competing risk analysis differs from the previously described time-to-event analysis in that significant differences were identified between groups when accounting for the competing risk of overall survival $(\mathrm{p}<0.05)$, in favour of PN.

\subsection{Secondary outcome - CKD}

Kaplan-Meier curve - CKD

Kaplan Meier curves were created to demonstrate risk of new onset CKD stratified by surgical treatment (Figure 4.16). A comparison of curves with the log-rank test demonstrated a benefit for PN in reducing the risk of developing new-onset CKD $(\mathrm{p}<0.05)$. 
Figure 4.16: Kaplan Meier curves stratified by surgical treatment for outcome of developing new-onset CKD.

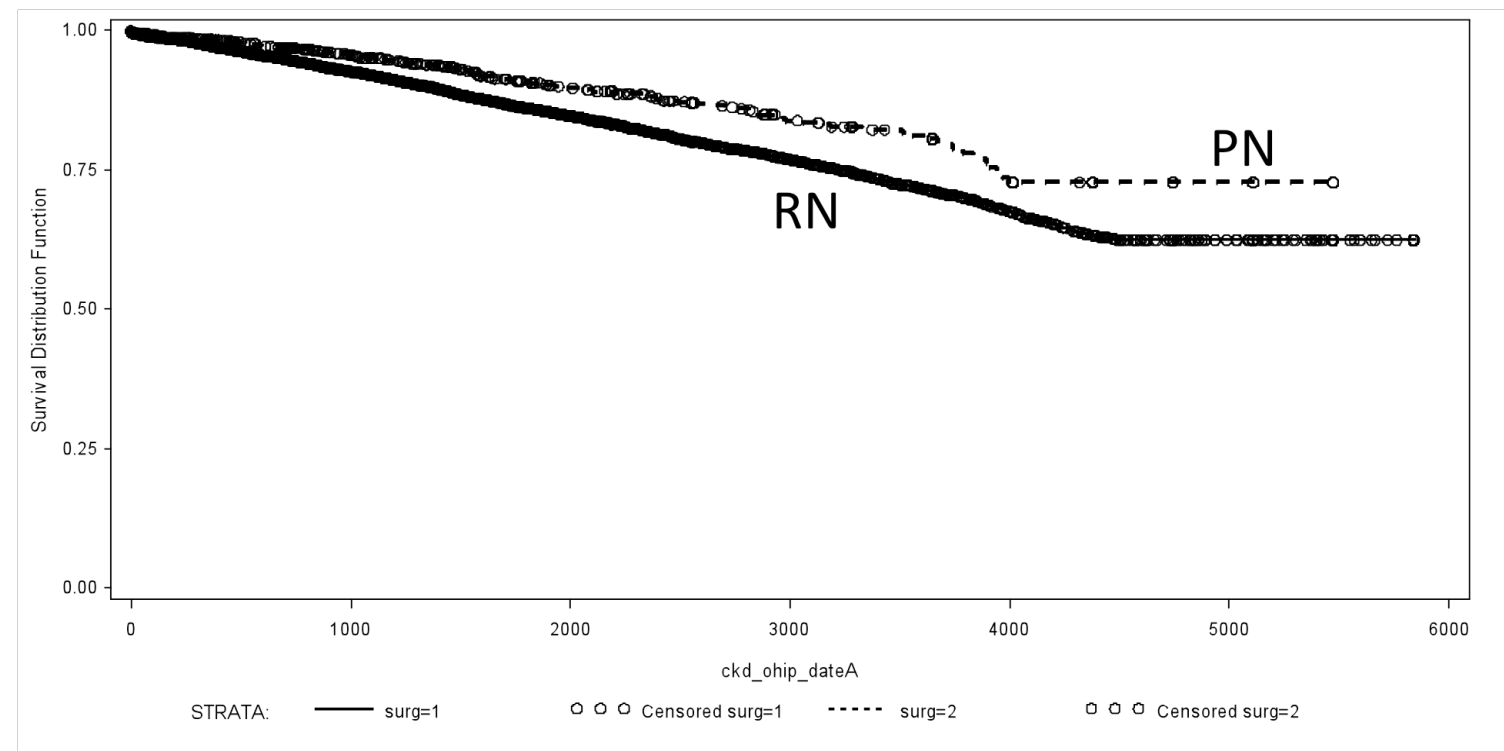

Cox proportional hazard model $-C K D$

Univariable analysis demonstrated that PN was associated with a decreased hazard of developing CKD (HR 0.66, 95\% CI 0.58-0.77). Other variables significant on univariable analysis included male gender (HR 1.48, 95\% CI 1.35-1.63), increasing patient age $(\mathrm{p}<0.05)$, recent diagnosis year group (HR 5.87, 95\% CI 5.01-6.87), histology (papillary (HR 1.29, 95\% CI 1.06-1.59) and unspecified histology (HR 0.73, 95\% CI 0.66-0.81)), pre-operative HTN (HR 1.83, 95\% CI 1.67-2.00), and pre-operative DM (HR $2.04,95 \%$ CI $1.85-2.26)$.

Variables that remained significant on multivariable analysis included having undergone PN (HR 0.48, 0.41-0.57), male gender (HR 1.58, 1.44-1.74), patient age $\geq 80$ (HR 6.45, 5.47-7.61), recent diagnosis year group (HR 6.45, 5.47-7.61), pre-operative HTN (HR 1.46, 95\% CI 1.33-1.60), and pre-operative DM (HR 1.67, 95\% CI 1.51-1.85) (Table 4.8). 
Table 4.8: Univariable and multivariable Cox proportional hazards model (new-onset CKD, $n=11,342)$

\begin{tabular}{|l|l|l|l|l|}
\hline & Univariable & & Multivariable & \\
\hline & HR (CI) & p-value & HR (CI) & p-value \\
\hline Surgery & & & & \\
\hline RN & Ref & & Ref & \\
\hline PN & $0.66(0.58-0.77)$ & $<0.05$ & $0.48(0.41-0.57)$ & $<0.05$ \\
\hline Sex & & & & \\
\hline Female & Ref & & Ref & \\
\hline Male & $1.48(1.35-1.63)$ & $<0.05$ & $1.58(1.44-1.74)$ & $<0.05$ \\
\hline Age group & & $<0.05$ & & $<0.05$ \\
\hline$<50$ & Ref & & Ref & \\
\hline $50-59$ & $1.53(1.30-1.79)$ & $<0.05$ & $1.32(1.12-1.55)$ & $<0.05$ \\
\hline $60-69$ & $2.29(1.98-2.66)$ & $<0.05$ & $1.87(1.61-2.17))$ & $<0.05$ \\
\hline $70-79$ & $2.87(2.45-3.34)$ & $<0.05$ & $2.40(2.06-2.81)$ & $<0.05$ \\
\hline$\geq 80$ & $4.20(3.43-5.15)$ & $<0.05$ & $6.45(5.47-7.61)$ & $<0.05$ \\
\hline Year group & & $<0.05$ & & $<0.05$ \\
\hline $1995-1999$ & Ref & & Ref & \\
\hline $2000-2004$ & $2.37(2.07-2.72)$ & $<0.05$ & $2.55(2.21-2.93)$ & $<0.05$ \\
\hline $2005-2010$ & $5.87(5.01-6.87)$ & $<0.05$ & $6.45(5.47-7.61)$ & $<0.05$ \\
\hline Histology & & $<0.05$ & & 0.43 \\
\hline Clear cell & Ref & & & \\
\hline Papillary & $1.29(1.06-1.59)$ & $<0.05$ & & \\
\hline Chromophobe & $1.05(0.81-1.36)$ & 0.68 & & \\
\hline NOS & $0.73(0.66-0.81)$ & $<0.05$ & & \\
\hline HTN & & & & $<0.05$ \\
\hline No & Ref & & Ref & \\
\hline Yes & $1.83(1.67-2.00)$ & $<0.05$ & $1.46(1.33-1.60)$ & \\
\hline MI & & & & \\
\hline No & Ref & & Ref & \\
\hline Yes & $1.44(0.99-2.09)$ & 0.05 & $1.29(0.88-1.87)$ & 0.17 \\
\hline DM & & & & Ref \\
\hline No & Ref & & $1.67(1.51-1.85)$ & $<0.05$ \\
\hline Yes & $2.04(1.85-2.26)$ & $<0.05$ & \\
\hline Abbrevatins & & & \\
\hline
\end{tabular}

Abbreviations: $\mathrm{HR}=$ hazards ratio; $\mathrm{CI}=$ confidence interval; $\mathrm{RN}=$ radical nephrectomy; $\mathrm{PN}=$ partial nephrectomy; NOS = not otherwise specified; $\mathrm{HTN}=$ hypertension; $\mathrm{MI}=$ myocardial infarction; $\mathrm{DM}=$ diabetes mellitus. 
Propensity score model - CKD

A significant benefit was identified for PN regarding the outcome of time to newonset CKD controlling for propensity score as a continuous variable (HR 0.50, 95\% CI 0.43-0.59). Similar results were demonstrated utilizing propensity score as a categorical variable (quintiles) (Appendix A, Table 4).

Competing risks analysis - CKD

Significant differences were demonstrated for the outcome of new-onset CKD on competing risks analysis controlling for overall mortality, with PN offering a significant protective effect $(\mathrm{p}<0.05)$. (Figure 4.17)

Figure 4.17: Competing risks analysis: cumulative probability curves stratified by surgical treatment for the outcome of the development of new-onset CKD.

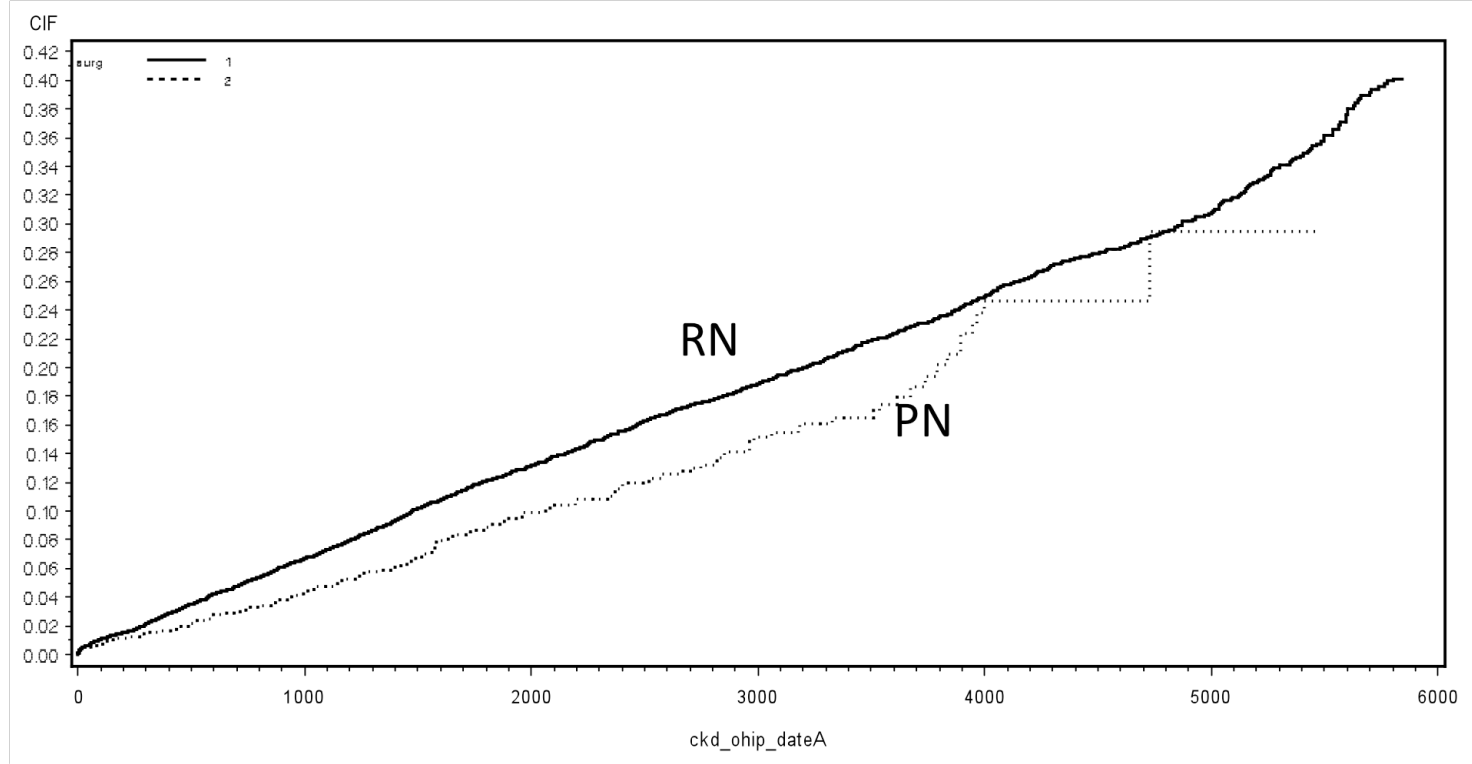




\section{Summary (new-onset CKD)}

Significant differences were identified between $\mathrm{RN}$ and $\mathrm{PN}$ regarding the outcome of new-onset CKD. A comparison of curves using the log-rank test demonstrated significant differences between the two surgical treatments groups $(\mathrm{p}<0.05)$. Significant differences were identified with a similar HR for both the multivariable Cox PH models after adjusting for individual comorbidities (HR 0.48, 0.41-0.57) and propensity score (HR $0.50,95 \%$ CI $0.43-0.59)$. Differences persisted between groups when accounting for the competing risk of overall survival $(\mathrm{p}<0.05)$.

\subsection{Secondary outcome - Acute dialysis}

Cox proportional hazards model - Acute dialysis

Univariable Cox proportional hazards analysis demonstrated that surgical approach was not significantly associated with time to acute dialysis (HR $1.14,95 \%$ CI $0.85-1.54)$. Other variables significant on univariable analysis included male gender (HR $1.43,95 \%$ CI $1.13-1.82)$, increasing patient age $(\mathrm{p}<0.05)$, recent diagnosis year group (HR 0.64, 95\% CI 0.47-0.85), unspecified histology (HR 1.34, 95\% CI 1.02-1.76), preoperative HTN (HR 1.45, 95\% CI 1.17-1.80), pre-operative DM (HR 2.19, 95\% CI 1.732.78), and pre-operative CKD (HR 2.19, 95\% CI 1.47-3.28).

Variables that remained significant on multivariable analysis included male gender (HR 1.29, 95\% CI 1.01-1.65), patient age $\geq 80$ (HR 1.95, 95\% CI 1.11-3.43), most recent diagnosis year group (HR 0.45, 0.32-0.63), pre-operative HTN (HR 1.40, 95\% CI 1.11-1.76), and pre-operative CKD (HR 10.08, 95\% CI 7.75-13.11) (Table 4.9). 
Table 4.9: Univariable and multivariable Cox proportional hazards model (acute dialysis)

\begin{tabular}{|c|c|c|c|c|}
\hline & Univariable & & Multivariable & \\
\hline & $\mathrm{HR}(\mathrm{CI})$ & p-value & $\mathrm{HR}(\mathrm{CI})$ & p-value \\
\hline \multicolumn{5}{|l|}{ Surgery } \\
\hline $\mathrm{RN}$ & Ref & & Ref & \\
\hline $\mathrm{PN}$ & $1.14(0.85-1.54)$ & 0.37 & $0.93(0.68-1.27)$ & 0.65 \\
\hline \multicolumn{5}{|l|}{ Sex } \\
\hline Female & Ref & & Ref & \\
\hline Male & $1.43(1.13-1.82)$ & $<0.05$ & $1.29(1.01-1.65)$ & $<0.05$ \\
\hline Age group & & $<0.05$ & & $<0.05$ \\
\hline$<50$ & Ref & & Ref & \\
\hline $50-59$ & $1.57(1.02-2.41)$ & $<0.05$ & $1.33(0.87-2.06)$ & 0.18 \\
\hline $60-69$ & $2.46(1.65-3.66)$ & $<0.05$ & $1.82(1.21-2.74)$ & $<0.05$ \\
\hline $70-79$ & $3.20(2.15-4.78)$ & $<0.05$ & $2.12(1.40-3.20)$ & $<0.05$ \\
\hline$\geq 80$ & $3.13(1.81-5.40)$ & $<0.05$ & $1.95(1.11-3.43)$ & $<0.05$ \\
\hline Year group & & $<0.05$ & & $<0.05$ \\
\hline $1995-1999$ & Ref & & Ref & \\
\hline $2000-2004$ & $0.85(0.65-1.12)$ & 0.25 & $0.75(0.56-1.00)$ & 0.05 \\
\hline $2005-2010$ & $0.64(0.47-0.85)$ & $<0.05$ & $0.45(0.32-0.63)$ & $<0.05$ \\
\hline Histology & & $<0.05$ & & 0.36 \\
\hline Clear cell & Ref & & & \\
\hline Papillary & $1.50(0.90-2.49)$ & 0.11 & & \\
\hline Chromophobe & $0.56(0.22-1.40)$ & 0.21 & & \\
\hline NOS & $1.34(1.02-1.76)$ & $<0.05$ & & \\
\hline \multicolumn{5}{|l|}{ HTN } \\
\hline $\mathrm{No}$ & Ref & & Ref & \\
\hline Yes & $1.90(1.51-2.37)$ & $<0.05$ & $1.40(1.11-1.76)$ & $<0.05$ \\
\hline \multicolumn{5}{|l|}{ MI } \\
\hline No & Ref & & Ref & \\
\hline Yes & $2.28(1.07-4.83)$ & 0.66 & $1.07(0.50-2.31)$ & 0.84 \\
\hline \multicolumn{5}{|l|}{$\mathrm{DM}$} \\
\hline No & Ref & & Ref & \\
\hline Yes & $3.01(2.39-3.79)$ & $<0.05$ & $2.12(1.67-2.70)$ & 0.05 \\
\hline \multicolumn{5}{|l|}{ CKD } \\
\hline No & Ref & & Ref & \\
\hline Yes & $6.82(4.96-9.37)$ & $<0.05$ & $10.08(7.75-13.11)$ & $<0.05$ \\
\hline
\end{tabular}

Abbreviations: $\mathrm{HR}=$ hazards ratio; $\mathrm{CI}=$ confidence interval; $\mathrm{RN}=$ radical nephrectomy; $\mathrm{PN}=$ partial nephrectomy; $\mathrm{NOS}=$ not otherwise specified; $\mathrm{HTN}=$ hypertension; $\mathrm{MI}=$ myocardial infarction; $\mathrm{DM}=$ diabetes mellitus; $\mathrm{CKD}=$ chronic kidney disease. 


\section{Propensity score model - Acute dialysis}

No significant differences were identified for the outcome of acute dialysis using a propensity score model (continuous variable) to control bias between groups (HR 1.13, 95\% CI 0.83-1.55). Similar results were demonstrated utilizing propensity score as a categorical variable (quintiles) (Appendix A, Table 4).

Competing risks analysis - Acute dialysis

No significant differences were identified for the outcome of acute dialysis, despite controlling for the competing risk of overall mortality $(\mathrm{p}=0.81)$. (Figure 4.18)

Figure 4.18: Competing risks analysis: cumulative probability curves stratified by surgical treatment for the outcome of acute dialysis

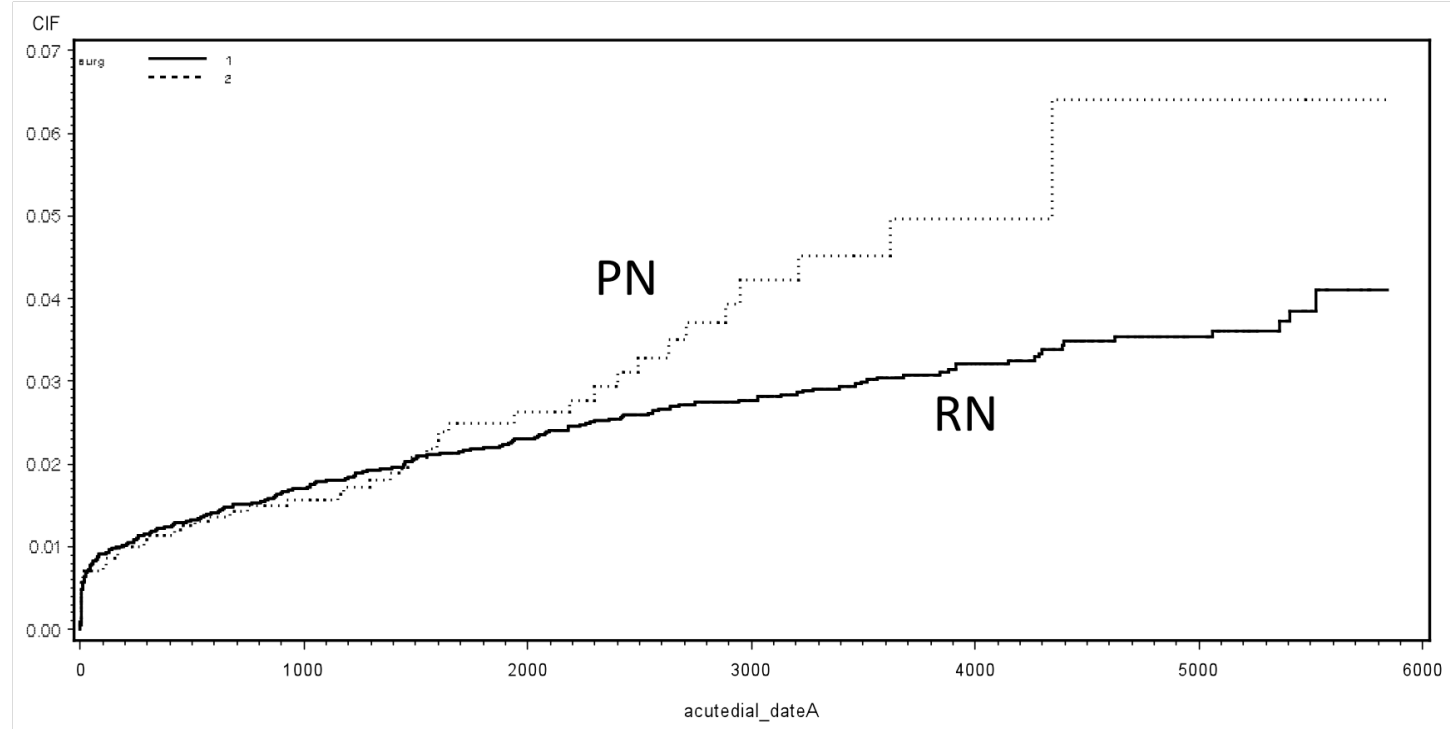


Summary (acute dialysis)

Significant differences were not identified between RN and PN regarding the outcome of acute dialysis. A comparison of groups did not identify significant differences when utilizing a multivariable Cox PH models adjusting for individual comorbidities (HR 1.29, 95\% CI 1.01-1.65) or propensity scores (HR 1.13, 95\% CI 0.83-1.55). Furthermore, no significant difference was identified between groups when accounting for the competing risk of overall survival $(\mathrm{p}=0.81)$.

\subsection{Secondary outcome - Overall survival}

Kaplan-Meier curve - Overall survival

Kaplan Meier curves were created to demonstrate risk of death stratified by surgical treatment (Figure 4.19). A comparison of curves with the log-rank test demonstrated a significant association between PN and improved overall survival $(p<0.05)$. Overall survival at 5 years was $65 \%$ for the entire cohort, with a 5-year overall survival of 59\% amongst those undergoing $\mathrm{RN}$, compared $90 \%$ among those undergoing PN. 
Figure 4.19: Kaplan Meier curves stratified by surgical treatment for outcome of overall survival.

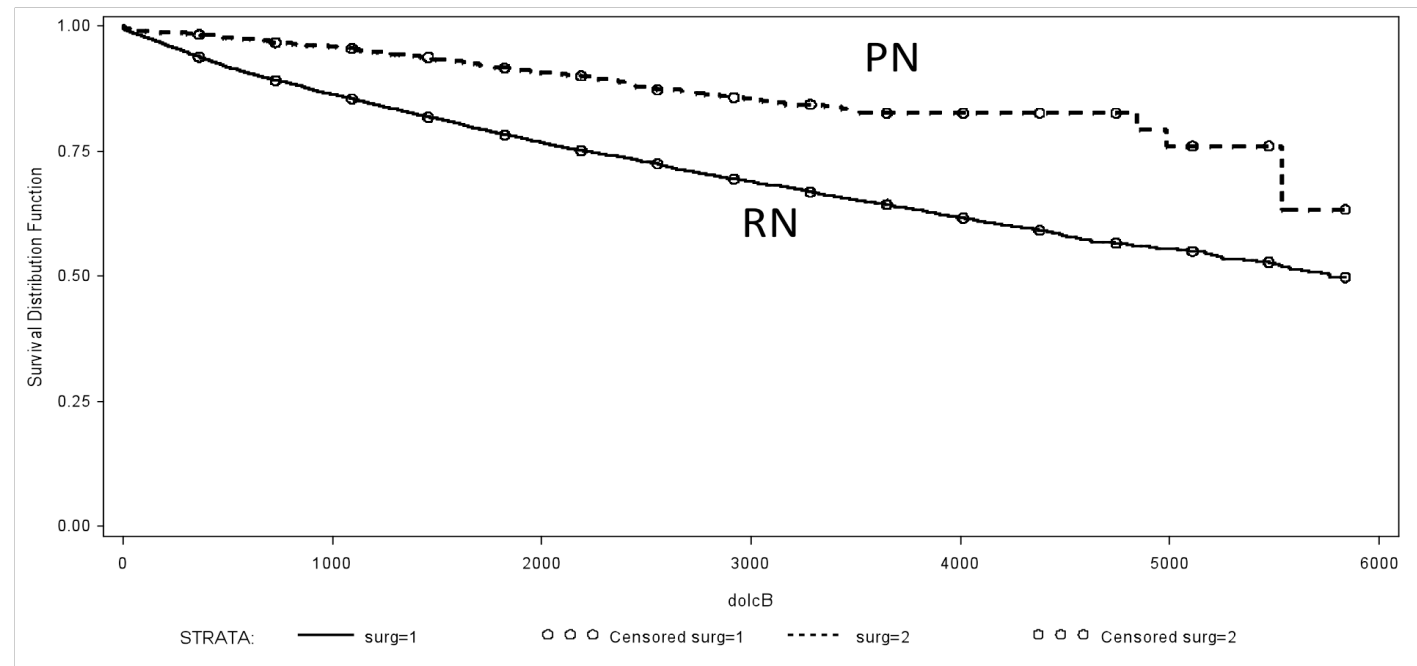

Cox proportional hazards model - Overall survival

Univariable Cox proportional hazards analysis demonstrated that PN, compared to RN, was associated with a decreased hazards of death (HR 0.37, 95\% CI 0.31-0.43). Other variables significant on univariable analysis included male gender (HR 1.25, 95\% CI 1.15-1.34), increasing age group $(\mathrm{p}<0.05)$, recent diagnosis year group (HR $0.64,95 \%$ CI 0.57-0.71), unspecified histology (HR 1.77, 95\% CI 1.61-1.96), pre-operative HTN (HR 1.18, 95\% CI 1.10-1.27), pre-operative MI (HR 1.79, 95\% CI 1.37-2.36), preoperative DM (HR 1.58, 95\% CI 1.45-1.72), and pre-operative CKD (HR 1.68, 95\% CI $1.32-2.14)$

Variables that remained significant on multivariable analysis included having undergone PN (HR 0.43, 95\% CI 0.37-0.51), male gender (HR 1.33, 95\% CI 1.23-1.43), patient age $\geq 80$ (HR 7.62, 95\% CI 6.46-8.98), recent diagnosis year group (HR 0.87, 95\% CI 0.78-0.98), unspecified histology (HR 1.66, 95\% CI 1.50-1.84), pre-operative MI (HR 
1.47, 95\% CI 1.12-1.93), pre-operative DM (HR 1.42, 95\% CI 1.30-1.55), and preoperative CKD (HR 1.49, 95\% CI 1.28-1.74) (Table 4.10).

Table 4.10: Univariable and multivariable Cox proportional hazards model (overall survival)

\begin{tabular}{|l|l|l|l|l|}
\hline & Univariable & & Multivariable & \\
\hline & HR (CI) & p-value & HR (CI) & p-value \\
\hline Surgery & & & & \\
\hline RN & Ref & & Ref & \\
\hline PN & $0.37(0.31-0.43)$ & $<0.05$ & $0.43(0.37-0.51)$ & $<0.05$ \\
\hline Sex & & & & \\
\hline Female & Ref & & Ref & \\
\hline Male & $1.25(1.15-1.34)$ & $<0.05$ & $1.33(1.23-1.43)$ & $<0.05$ \\
\hline Age group & & $<0.05$ & & $<0.05$ \\
\hline$<50$ & Ref & & Ref & \\
\hline $50-59$ & $1.79(1.54-2.08)$ & $<0.05$ & $1.73(1.48-2.02)$ & $<0.05$ \\
\hline $60-69$ & $2.81(2.44-3.24)$ & $<0.05$ & $2.65(2.28-3.04)$ & $<0.05$ \\
\hline $70-79$ & $4.45(3.87-5.12)$ & $<0.05$ & $4.19(3.63-4.83)$ & $<0.05$ \\
\hline$\geq 80$ & $8.22(6.99-9.66)$ & $<0.05$ & $7.62(6.46-8.98)$ & $<0.05$ \\
\hline Year group & & $<0.05$ & & $<0.05$ \\
\hline $1995-1999$ & Ref & & Ref & \\
\hline $2000-2004$ & $0.80(0.74-0.88)$ & $<0.05$ & $0.99(0.91-1.09)$ & 0.96 \\
\hline $2005-2010$ & $0.64(0.57-0.71)$ & $<0.05$ & $0.87(0.78-0.98)$ & $<0.05$ \\
\hline Histology & & $<0.05$ & & $<0.05$ \\
\hline Clear cell & Ref & & Ref & \\
\hline Papillary & $0.86(0.67-1.09)$ & 0.22 & $0.86(0.67-1.09)$ & 0.23 \\
\hline Chromophobe & $0.82(0.61-1.10)$ & 0.19 & $0.95(0.71-1.27)$ & 0.76 \\
\hline NOS & $1.77(1.61-1.96)$ & $<0.05$ & $1.66(1.50-1.84)$ & $<0.05$ \\
\hline HTN & & & & \\
\hline No & Ref & & Ref & \\
\hline Yes & $1.18(1.10-1.27)$ & $<0.05$ & $0.92(0.86-1.00)$ & 0.05 \\
\hline MI & & & & \\
\hline No & Ref & & Ref & \\
\hline Yes & $1.79(1.37-2.36)$ & $<0.05$ & $1.47(1.12-1.93)$ & $<0.05$ \\
\hline DM & & & & \\
\hline No & Ref & & Ref & \\
\hline Yes & $1.58(1.45-1.72)$ & $<0.05$ & $1.42(1.30-1.55)$ & $<0.05$ \\
\hline CKD & Ref & & Ref & \\
\hline No & $1.68(1.32-2.14)$ & $<0.05$ & $1.49(1.28-1.74)$ & $<0.05$ \\
\hline Yes & & & & \\
\hline & & & \\
\hline
\end{tabular}


Abbreviations: $\mathrm{HR}=$ hazards ratio; $\mathrm{CI}=$ confidence interval; $\mathrm{RN}=$ radical nephrectomy; $\mathrm{PN}=$ partial nephrectomy; $\mathrm{NOS}=$ not otherwise specified; HTN = hypertension; $\mathrm{MI}=$ myocardial infarction; $\mathrm{DM}=$ diabetes mellitus; $\mathrm{CKD}=$ chronic kidney disease.

\section{Propensity score model - Overall survival}

PN was associated with a decreased risk of overall mortality compared to RN (HR 0.45, 95\% CI $0.38-0.52$ ) utilizing propensity score as a continuous variable to control for confounding factors. Similar results were demonstrated utilizing propensity score as a categorical variable (quintiles) (Appendix A, Table 4).

\section{Summary (Overall survival)}

Significant differences were identified between RN and PN regarding the outcome of overall survival. A comparison of curves using the log-rank test demonstrated significant differences between the two surgical treatments groups $(\mathrm{p}<0.05)$. Significant differences were identified with a similar HR on both the multivariable Cox PH models adjusting for individual comorbidities (HR 0.43, 95\% CI 0.37-0.51) and propensity score (HR 0.45, $95 \%$ CI $0.38-0.52)$.

\subsection{Secondary outcome - Cancer-specific survival}

Kaplan-Meier curve - Cancer-specific survival

Kaplan Meier curves were created to demonstrate risk of cancer-specific death stratified by surgical treatment (Figure 4.20). A comparison of curves with the log-rank test demonstrated a significant association between PN and improved cancer-specific survival $(\mathrm{p}<0.05)$. Cancer-specific survival at 5 years was $84 \%$ for the entire cohort, with 
a 5-year cancer-specific survival of $80 \%$ amongst those undergoing $\mathrm{RN}$, compared to 98\% among those undergoing PN.

Figure 4.20: Kaplan Meier curves stratified by surgical treatment for outcome of cancerspecific survival.

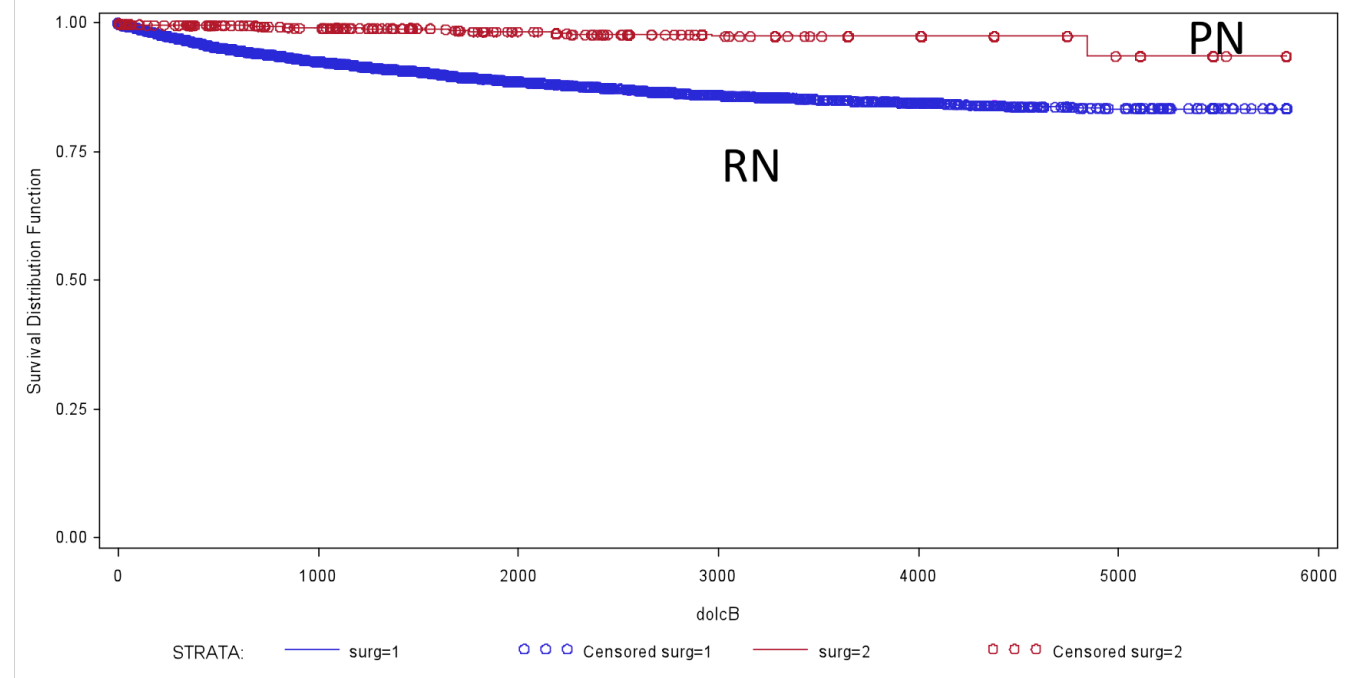

Cox proportional hazard model-Cancer-specific survival

Univariable Cox proportional hazards analysis demonstrated that PN was associated with a decreased hazard of cancer-specific death (HR 0.14, 95\% CI 0.10-0.21). Other variables significant on univariable analysis included male gender (HR 1.27, 95\% CI 1.12-1.43), increasing patient age $(<0.05)$, recent diagnosis year group $($ HR $0.25,95 \%$ 
CI 0.21-0.30), unspecified histology (HR 2.82, 95\% CI 2.37-3.35), and pre-operative HTN (HR 0.87, 95\% CI 0.77-0.99).

Variables that remained significant on multivariable analysis included having undergone PN (HR 0.21, 95\% CI 0.14-0.30), male gender (HR 1.33, 95\% CI 1.18-1.51), patient age $\geq 80$ (HR 4.13, 95\% CI 3.21-5.32), recent diagnosis year group (HR 0.42, 95\% CI 0.35-0.51), unspecified histology (HR 2.16, 95\% CI 1.80-2.59), pre-operative HTN (HR 0.79, 95\% CI 0.70-0.90), and pre-operative CKD (HR 1.85, 95\% CI 1.36-2.49) (Table 4.11). 
Table 4.11: Univariable and multivariable Cox proportional hazards model (cancerspecific survival)

\begin{tabular}{|c|l|l|l|l|}
\hline & Univariable & & Multivariable & \\
\hline & HR (CI) & p-value & HR (CI) & p-value \\
\hline Surgery & & & & \\
\hline RN & Ref & & Ref & \\
\hline PN & $0.14(0.10-0.21)$ & $<0.05$ & $0.21(0.14-0.30)$ & $<0.05$ \\
\hline Sex & & & & \\
\hline Female & Ref & & Ref & \\
\hline Male & $1.27(1.12-1.43)$ & $<0.05$ & $1.33(1.18-1.51)$ & $<0.05$ \\
\hline Age group & & $<0.05$ & & $<0.05$ \\
\hline$<50$ & Ref & & Ref & \\
\hline $50-59$ & $1.51(1.22-1.87)$ & $<0.05$ & $1.54(1.25-1.91)$ & $<0.05$ \\
\hline $60-69$ & $1.94(1.58-2.37)$ & $<0.05$ & $1.92(1.56-2.36)$ & $<0.05$ \\
\hline $70-79$ & $2.49(2.03-3.05)$ & $<0.05$ & $2.45(1.99-3.02)$ & $<0.05$ \\
\hline$\geq 80$ & $4.16(3.25-5.32)$ & $<0.05$ & $4.13(3.21-5.32)$ & $<0.05$ \\
\hline Year group & & $<0.05$ & & $<0.05$ \\
\hline $1995-1999$ & Ref & & Ref & \\
\hline $2000-2004$ & $0.67(0.58-0.76)$ & $<0.05$ & $0.92(0.80-1.05)$ & 0.24 \\
\hline $2005-2010$ & $0.25(0.21-0.30)$ & $<0.05$ & $0.42(0.35-0.51)$ & $<0.05$ \\
\hline Histology & & $<0.05$ & & $<0.05$ \\
\hline Clear cell & Ref & & Ref & \\
\hline Papillary & $0.72(0.45-1.15)$ & 0.17 & $0.82(0.51-1.31)$ & 0.40 \\
\hline Chromophobe & $0.88(0.53-1.45)$ & 0.62 & $1.08(0.66-1.80)$ & 0.74 \\
\hline NOS & $2.82(2.37-3.35)$ & $<0.05$ & $2.16(1.80-2.59)$ & $<0.05$ \\
\hline HTN & & & & \\
\hline No & Ref & & Ref & \\
\hline Yes & $0.87(0.77-0.99)$ & $<0.05$ & $0.79(0.70-0.90)$ & $<0.05$ \\
\hline MI & & & & \\
\hline No & Ref & & Ref & \\
\hline Yes & $1.12(0.65-1.95)$ & 0.66 & $0.91(0.53-1.58)$ & 0.75 \\
\hline DM & & & & \\
\hline No & Ref & & Ref & \\
\hline Yes & $1.14(0.98-1.32)$ & 0.07 & $1.16(0.99-1.35)$ & 0.05 \\
\hline CKD & Ref & & & Ref \\
\hline No & $0.98(0.73-1.31)$ & 0.89 & $1.85(1.36-2.49)$ & $<0.05$ \\
\hline Yes & & & & \\
\hline & & & \\
\hline
\end{tabular}

Abbreviations: $\mathrm{HR}=$ hazards ratio $\mathrm{CI}=$ confidence interval; $\mathrm{RN}=$ radical nephrectomy; $\mathrm{PN}=$ partial nephrectomy; NOS = not otherwise specified; HTN = hypertension; $\mathrm{MI}=$ myocardial infarction; $\mathrm{DM}=$ diabetes mellitus; $\mathrm{CKD}=$ chronic kidney disease. 
Propensity score model - Cancer specific survival

PN was associated with a decreased risk of cancer-specific mortality compared to RN (HR $0.21,95 \%$ CI $0.15-0.31$ ) utilizing propensity score as a continuous variable to control for confounding factors. Similar results were demonstrated utilizing propensity score as a categorical variable (quintiles) (Appendix A, Table 4).

\section{Summary (Cancer specific survival)}

Significant differences were identified between RN and PN regarding the outcome of cancer-specific survival. A comparison of curves using the log-rank test demonstrated significant differences between the two surgical treatments groups $(\mathrm{p}<0.05)$. Significant differences were identified with a similar HR on both the multivariable Cox PH models adjusting for individual comorbidities (HR 0.21, 95\% CI 0.14-0.30) and propensity score (HR 0.21, 95\% CI 0.15-0.31).

\subsection{Summary of Findings}

No significant benefit was identified for PN compared to $\mathrm{RN}$ in preventing the onset of ESRD requiring RRT in the primary analysis of the overall cohort. However, when data were analyzed for the modern cohort (2003-2010), a significant benefit was identified for PN compared to RN. Similar findings were demonstrated using propensity score analysis to control for confounding between the groups in this modern cohort. A benefit for undergoing PN compared to RN was demonstrated for the prevention of newonset $\mathrm{CKD}$ as well as for overall survival utilizing multivariable time-to-event analysis. A benefit of PN regarding hospitalization for MI was not identified on time-to-event 
analysis, but was demonstrated once controlling for competing risks of death. A summary of findings for all outcomes is displayed in Table 4.12.

Table 4.12: Summary of findings for the impact of PN compared to RN across various outcomes and analysis methods.

\begin{tabular}{|l|l|ll|ll|l|}
\hline & Log rank & \multicolumn{2}{|l|}{ Cox PH model } & \multicolumn{2}{l|}{ Propensity score } & $\begin{array}{l}\text { Competing } \\
\text { risks }\end{array}$ \\
\cline { 2 - 7 } & p-value & HR & $95 \%$ CI & HR & $95 \%$ CI & p-value \\
\hline $\begin{array}{l}\text { ESRD } \\
\text { (overall) }\end{array}$ & 0.62 & 0.75 & $0.54-1.05$ & 1.04 & $0.75-1.45$ & 0.37 \\
\hline $\begin{array}{l}\text { ESRD } \\
\text { (modern cohort) }\end{array}$ & $<0.05$ & 0.44 & $0.25-0.75$ & 0.47 & $0.27-0.82$ & $<0.05$ \\
\hline $\begin{array}{l}\text { Myocardial } \\
\text { infarction }\end{array}$ & $<0.05$ & 0.76 & $0.52-1.12$ & 0.81 & $0.55-1.19$ & $<0.05$ \\
\hline New-onset CKD & $<0.05$ & 0.48 & $0.41-0.57$ & 0.50 & $0.43-0.59$ & $<0.05$ \\
\hline Acute dialysis & 0.37 & 0.93 & $0.68-1.27$ & 1.13 & $0.83-1.55$ & 0.81 \\
\hline Overall survival & $<0.05$ & 0.43 & $0.37-0.51$ & 0.45 & $0.38-0.52$ & $\mathrm{n} / \mathrm{a}$ \\
\hline $\begin{array}{l}\text { Cancer-specific } \\
\text { survival }\end{array}$ & $<0.05$ & 0.21 & $0.14-0.30$ & 0.21 & $0.15-0.31$ & $\mathrm{n} / \mathrm{a}$ \\
\hline
\end{tabular}

Abbreviations: $\mathrm{PH}=$ proportional hazards; $\mathrm{HR}=$ hazards ratio; $\mathrm{ESRD}=$ end stage renal disease; $\mathrm{CKD}=$ chronic kidney disease, $\mathrm{n} / \mathrm{a}=$ not applicable. 


\section{CHAPTER 5 DISCUSSION}

\subsection{Summary}

The current study compared outcomes of PN and RN for the treatment of RCC using administrative databases from the province of Ontario. Significant benefits were not identified for PN in the prevention of ESRD when the entire cohort was analyzed. However, a significant benefit was identified for PN when analyzing a subgroup of patients diagnosed in a modern cohort (2003-2010), despite controlling for confounding factors and accounting for competing risks. Compared to RN, PN was also associated with the prevention of new-onset CKD, decreased rates of MI, and improved overall survival. Lastly, we reported updated trends in the surgical management of RCC and demonstrated continued uptake of PN across the province.

\subsection{Primary outcome - ESRD}

Surgical management of patients with RCC with PN was associated with decreased rates of ESRD requiring RRT compared to RN. This was not seen when analyzing the cohort across all years, but was demonstrated with a strong association (HR 0.44, CI 0.25-0.75) in the modern cohort of patients (2003-2010). Analysis of the entire cohort demonstrated a trend towards a benefit for PN in the prevention of ESRD (HR 0.75, CI 0.54-1.05) though findings fell short of statistical significance. The benefit of PN compared to $\mathrm{RN}$ with regards to ESRD in the modern cohort was a robust finding that remained consistent across various methods of analysis and approaches of controlling for confounders. Furthermore, analysis was performed in sequential cohorts based on year of diagnosis with 2003 identified as an important time point after which the benefit of PN in 
preventing ESRD was found to be statistically significant. In each subsequent cohort more recent than 2003, this association was identified with increasing strength (Table 4.6).

The distinction of a modern cohort is important since it consists of patients that encompass a more accurate representation of current practice. Prior to 2003, PN was not universally accepted as a standard of care for the treatment of small renal masses and broader uptake of this procedure did not occur until the early $2000 \mathrm{~s} .{ }^{82}$ The potential selection bias present in the earlier years of this cohort may make such comparisons between the groups difficult, especially regarding outcomes of renal function. During earlier years of the present cohort, PN had yet to gain widespread use and acceptance. PN was primarily reserved for patients with concerns for impending ESRD including those with solitary kidneys, bilateral tumours, or pre-existing CKD. Its utilization was primarily in select groups of patients and concentrated in academic institutions, as opposed to being performed broadly across various practice settings as it is today. Including such patients in an analysis of renal functional outcomes will bias findings towards a benefit for RN compared to PN, and furthermore would not accurately reflect current practice patterns.

In addition, we demonstrated low rates of PN and widely variable incidence rates of ESRD among patients undergoing surgery in the initial years of our cohort. PN constituted the surgical approach for $8.3 \%$ of patients undergoing renal surgery for RCC between 1995 and 2002, with absolute yearly numbers of cases performed increasing from 7 in 1995 to 116 in 2002.

These findings represent the first demonstration of the impact of surgical treatments for RCC on the subsequent development of ESRD requiring RRT. Though 
previous work has demonstrated the impact of surgical approach on estimates of renal function $^{2,137}$, this is the first demonstration of hard outcomes of renal failure. This is an important finding in and of itself, given the current lack of data in this aspect of the literature along with the continuing debate regarding the overall benefit of PN compared to $\mathrm{RN}$ across a spectrum of outcomes.

\section{Trends in incidence of ESRD}

Through analysis of provincial data across a 15-year time frame, important population-level trends were identified regarding the development of ESRD in this population of surgically treated patients. The overall incidence of post-operative, newonset ESRD in our cohort was 2.5\%. Prior population-level studies of the general population have reported a lifetime risk of ESRD at $2.7 \%$ amongst men and $1.8 \%$ amongst women, which is consistent with findings from the current study. ${ }^{138}$

Another interesting finding is the decreasing rate of progression to ESRD among patients undergoing PN as we move across the analyzed time period (1995-2010). This shift likely represents the changing indications for performing PN. Initially, PN was considered only in extreme cases of impending ESRD, and it was utilized primarily as a final attempt to render the patient tumour-free while preserving enough renal function to avoid dialysis. PN rates were very low during the initial years of our study, as it was not a widely accepted standard of care and not the universally accepted first line treatment at the time. As we move towards the more modern years of the cohort, utilization of PN increased and the rates of ESRD began to stabilize. Only 76 (7.6\%) of the total PN cases 
were performed in the first 5 years of the cohort (1995-1999) and incidence rates of ESRD requiring dialysis in this subset was $4 \%$.

\subsection{Secondary outcomes}

We also present findings from multiple secondary outcomes that further contribute to the overall understanding of the consequences of surgical treatments for RCC. Many of these outcomes have been previously assessed in prior retrospective studies as well as in the sole randomized clinical trial. The retrospective data have almost universally supported PN over RN for its benefits in improving overall survival, reducing cardiovascular morbidity, and preserving renal function. The results of the EORTC trial contradict some of these findings in that it demonstrated a benefit of $\mathrm{RN}$ compared to $\mathrm{PN}$ in overall survival. Intention-to-treat analysis demonstrated a 10 -year overall survival rate of $81.1 \%$ for $\mathrm{RN}$ and $75.7 \%$ for $\mathrm{PN}$ (HR 1.50,95\% CI 1.03-2.16). Debate remains as to the true impact of these surgical choices and our data lend further support to understanding this controversy by analysis of a large population-based cohort with comprehensive and long follow-up, while also accounting for differences in comorbidity between surgical treatment groups and competing risks of mortality.

\subsubsection{Chronic kidney disease (CKD)}

We demonstrated a clear benefit of $\mathrm{PN}$ compared to $\mathrm{RN}$ in preserving renal function, as measured by new diagnoses of $\mathrm{CKD}$. The protective effects of $\mathrm{PN}$ were robust and consistent when analyzed utilizing multivariable Cox PH models (HR 0.48, 0.41-0.57) and propensity score modeling (HR 0.50, 0.43-0.59) as methods to control for 
bias between the groups. These findings lend further evidence to the benefits of nephron preservation in regards to subsequent renal function. Such findings are important in that the impact on renal function is arguably the most intuitive finding as well as that most directly related to the surgical insult.

The link between surgical approach and subsequent renal function has previously been explored in the urologic literature. Huang et al. in 2006 compared patients with normal contralateral kidneys and normal serum creatinine undergoing either RN or PN for a SRM. They assessed renal functional outcomes using the MDRD equation as a measure of eGFR. They found significant differences between the groups with a 3-year freedom from new-onset GFR lower than $60 \mathrm{ml} / \mathrm{min}$ per $1.73 \mathrm{~m}(2)$ of $80 \%$ after PN and $35 \%$ after RN. ${ }^{2}$ In another single institution series, RN compared to PN was associated with increased risk of renal functional decline across various measures including newonset CKD, percent change in eGFR, and change in CKD stage. ${ }^{139}$ Of the 209 patients with normal pre-operative eGFR undergoing surgery, 95/133 (71.4\%) of those undergoing RN and 13/76 (17.1\%) of those undergoing PN developed new onset CKD (eGFR $<60 \mathrm{~mL} / \mathrm{min}$ per $1.73 \mathrm{~m}(2)$ ). After controlling for demographic information and renal comorbidity (HTN, DM, CKD), RN (compared to PN) was found to be an independent predictor for their various methods of assessing change in renal function. Yet another study evaluated this question by measuring outcomes of serum creatinine, eGFR, acidosis, and proteinuria. This study again confirmed PN as an independent predictor of better renal function compared to $\mathrm{RN}$ across all pre-determined outcome measures. Their analysis of 749 patients undergoing either $\mathrm{RN}$ or PN demonstrated rates of new-onset CKD (eGFR $<60 \mathrm{~mL} / \mathrm{min}$ per $1.73 \mathrm{~m}(2)$ ) of $44.7 \%$ among those undergoing 
RN compared to $16 \%$ among those undergoing PN. RN was also identified as an independent risk factor $(\mathrm{HR} 3.08, \mathrm{p}<0.001)$ for the development of an elevated serum creatinine level $(\geq 2.0 \mathrm{mg} / \mathrm{dL})$.Other independent risk factors for decline in renal function determined by this study included a pre-operative diagnosis of DM, HTN, or CKD. ${ }^{140}$

These and other studies lay the foundation for developing the association between PN and improved renal function. The current study, however, offers several important advantages and insights when comparing its results to the previous literature. The majority of prior studies were from single institutional series. The current study confirms findings from a population-level analysis across all age groups, thus better characterizing real world outcomes. In addition, the current study is able to assess and control for comorbidity at levels comparable to institutional (clinical) databases, despite utilization of population-level data. Another unique component of the current study is its use of diagnoses of CKD and the development of ESRD as distinct clinical endpoints. This contrasts the previous literature, which historically utilized serum creatinine levels or measures of eGFR to assess renal outcomes. The "hard" outcomes utilized in the current study offer a unique approach to renal functional outcomes and potentially represent more clinically relevant outcomes.

\subsubsection{CKD and adverse outcomes}

Outcome measures of $\mathrm{CKD}$ are especially relevant because renal function serves an important mechanistic role in the development of other adverse outcomes including cardiac morbidity, overall survival, and the development of ESRD. The development of CKD may, in fact, represent an initial marker for the subsequent development of other 
morbidity and adverse outcome. The relationship between CKD and the development of adverse outcome and increased mortality has been well established in the medical literature and amongst patient populations with medically induced CKD. Go et al. published one of the initial studies reporting this relationship in 2004, and demonstrated the association between worsening renal function and increasing risks of hospitalization, cardiac events, and death. Estimated glomerular filtration rate (eGFR) was calculated in a cohort of over 1,000,000 patients who were then followed for approximately 3 years to assess outcomes. After controlling for confounding factors, an independent, step-wise association was identified between decreasing eGFR and adverse outcomes of hospitalization, cardiac events, and death. ${ }^{4}$ For example, as eGFR decreased from $\geq 60$ $\mathrm{mL} / \mathrm{min}$ per $1.73 \mathrm{~m}(2)$ to $<15 \mathrm{~mL} / \mathrm{min}$ per $1.73 \mathrm{~m}(2)$, the age-standardized rate of death from any cause (per 100 person-years) increased from 0.76 to 14.14 . Similarly, as eGFR decreased from $\geq 60 \mathrm{~mL} / \mathrm{min}$ per $1.73 \mathrm{~m}(2)$ to $<15 \mathrm{~mL} / \mathrm{min}$ per $1.73 \mathrm{~m}(2)$, the agestandardized rate of cardiovascular events (per 100 person-years) increased from 2.11 to 36.60.

The impact of CKD on adverse outcomes was further confirmed through a series of studies analyzing various populations, outcome measures, and methods of estimating kidney function. A large meta-analysis assessing over 2 million patients across various populations demonstrated a significant relationship between worsening renal function (reduced eGFR, increased albuminuria) and increasing risk of mortality and ESRD. ${ }^{141}$ Turin et al. utilized administrative databases in the province of Alberta to demonstrate an association between decreased eGFR and shorter life expectancy, ${ }^{142}$ while others demonstrated an association between worsening renal function (reduced eGFR, presence 
of proteinuria) and decreased overall survival, increased rates of MI, and increased likelihood of progression to ESRD. ${ }^{143}$

Similar relationships were demonstrated within subgroups with variable risks of developing CKD. A step-wise relationship was demonstrated between worsening renal function (decreased eGFR, increased albuminuria) and all-cause mortality, cardiovascular mortality, and development of ESRD regardless of hypertensive status. ${ }^{144}$ A similar study demonstrated an increased risk of ESRD and all-cause or cardiovascular death, regardless of whether or not a patient had a diagnosis of DM. ${ }^{145}$ Decreased eGFR remained a strong risk factor for the development ESRD and death despite specifically controlling for HTN and DM. ${ }^{146,147}$ These studies demonstrate that regardless of overall risk profile or the medical etiology of renal dysfunction, patients with CKD are at increased risk of adverse outcomes and should thus be monitored and managed appropriately. A body of literature has now established the relationship between worsening renal function and outcomes of cardiac morbidity and mortality, overall mortality, and the development of ESRD.

\subsubsection{Medical versus Surgical CKD}

The relationship between worsening renal function and the development of subsequent adverse outcome has been well established amongst patients with medicallyinduced renal dysfunction. Patients from the current study possess many of the previously discussed medically-related risk factors, yet potentially possess an additional surgical insult to their renal function. The role of surgically-induced renal injury is not as well understood as an independent risk factor for adverse outcome, and these patients may, in fact, behave differently over time than those with medically-induced renal dysfunction. In 
a recent paper, Lane et al. addressed this issue and attempt to distinguish between medically- and surgically-induced CKD. ${ }^{148}$ They demonstrated that surgical approach ( $\mathrm{RN}$ compared to $\mathrm{PN}$ ) may play a less significant role in predicting progressive deterioration of renal function amongst patients without pre-existing CKD. In an analysis of their single institutional cohort, patients with pre-existing CKD had a greater propensity for decline in their renal function following a surgical insult. In turn, those without significant CKD receiving only a surgical insult had a significantly lower risk of progressive renal dysfunction. They characterized the annual median renal functional decline (percent decrease in GFR) as $4.7 \%$ for those with medical CKD compared to $0.7 \%$ for those with surgical CKD $(\mathrm{p}<0.001)$. This study further emphasizes the importance of risk stratification to determine which patients will benefit the most from NSS. Although the current study does not directly address the question of the independent risks of surgically versus medically-induced renal injury, foundations are laid for future research in this critical area of study. Current findings further emphasize the risk of new-onset CKD in patients with medical comorbidity and demonstrate such behaviour in a surgical population.

\subsubsection{Myocardial infarction}

No significant differences were identified between surgical treatment groups regarding the incidence of myocardial infarction on initial time to event analysis. However, the HR was suggestive of a benefit for the prevention of MI in patients who underwent PN, and similar findings were identified whether comorbidity adjustment was performed utilizing a Cox PH model (HR $0.76,95 \%$ CI $0.52-1.12$ ) or propensity score 
model (HR 0.81, 95\% CI 0.55-1.19). Furthermore, a significant benefit in favour of PN in reducing the risk of MI was demonstrated after controlling for the competing risk of overall mortality $(\mathrm{p}<0.05)$. In a cohort where the population is relatively frail and elderly, such as those diagnosed with RCC, other failures (i.e. mortality) may occur prior to the outcome of interest (MI), thus masking the impact of a selected intervention (such as PN). Such competing risks may represent a significant bias and thus must be accounted for in the analysis. Cardiac outcomes from the current study support the benefits of PN in the treatment of RCC and also stress the importance of addressing competing risks in this analysis because of the many inter-related outcomes in this story.

The literature regarding outcomes of cardiac morbidity is less consistent, though findings generally point toward a benefit for PN. In 2009, Huang et al. published their results from the SEER database evaluating 1,991 patients with T1a renal tumours $(<4 \mathrm{~cm})$. In their study, $\mathrm{RN}$ was associated with a 1.4 times greater number of cardiac events. $\mathrm{RN}$, however, was not associated with time to first cardiac event or cardiac death. ${ }^{3}$ Miller et al. assessed 10,123 patients from a SEER-Medicare linked database and found no differences between $\mathrm{RN}$ and PN regarding cardiac events. ${ }^{149}$ Although an association between surgical approach and cardiac morbidity has been demonstrated, the links are not as robust or consistent as the associations between $\mathrm{RN}$ and $\mathrm{CKD}$ or between CKD and cardiac morbidity. Competing risks analysis was not performed in the majority of prior studies, thus potentially masking the true impact of surgical approach on cardiac outcomes. In the current study, differences between the surgical groups regarding MI were not statistically apparent until a competing risks analysis accounting for overall mortality was performed, although the HR was suggestive of a possible relationship but 
with a wide confidence interval. Thus, the effects of using a competing risks approach may have been to improve the precision of the estimate by reducing the 'noise' of competing outcomes (i.e. death).

\subsubsection{Acute dialysis}

No significant differences were identified between the surgical treatment groups regarding incidence of acute dialysis despite controlling for confounding factors and competing risks. This contrasts with findings from the same cohort of an independent association between PN and improved outcomes of long-term renal function, including diagnoses of CKD and development of ESRD. Although a body of literature has developed regarding outcomes based on serum creatinine and eGFR, studies have not been published to assess the impact of $\mathrm{RN}$ on hard outcomes of renal function including acute and chronic dialysis. Thus we provide the first data assessing such outcomes in patients undergoing either RN or PN.

Many studies have also developed the link between acute kidney injury (AKI) and long-term outcomes of renal function including CKD and ESRD. The majority of this work evaluates patients with medically-related renal risk factors and medically-induced CKD. A large meta-analysis identified AKI as an independent risk factor for the development of CKD (HR 8.8), ESRD (HR 3.1), and mortality (HR 2.1). ${ }^{150}$ Further studies assessed medical patients requiring acute dialysis in the ICU setting and demonstrated that, amongst those who survive beyond 1 year, $12.5 \%$ were dialysisdependant and between 19\% and 35\% developed new-onset CKD. ${ }^{151}$ Similarly poor outcomes have also been demonstrated in patients developing AKI after undergoing RN. 
Cho et al. reported their retrospective series of 519 patients with normal pre-operative renal function undergoing $\mathrm{RN}$ and identified a 4.2 fold increased risk of developing $\mathrm{CKD}$ $\left(\right.$ eGFR $<60 \mathrm{ml} / \mathrm{min} / 1.73 \mathrm{~m}^{2}$ ) if the patient suffered an episode of post-operative AKI. ${ }^{152}$

Despite a series of studies reporting the relationship between AKI and the subsequent development of CKD and ESRD, it remains difficult to isolate the individual impact of medical versus surgical renal injury. It may not be appropriate to make direct inferences from the medical literature to a population of patients suffering a surgical insult to their renal function such as those undergoing RN or PN. Such a relationship may not be as strong in the surgical population given their decreased propensity for progressive renal dysfunction, since much of the insult to the kidney occurred over a brief time point rather than continuously.

Results of the current study are inconsistent with respect to acute and chronic dialysis outcomes, with a clear benefit for PN in preventing the development of CKD and ESRD, yet no significant impact on the outcome of acute dialysis. There are some possible explanations for this discrepancy. Overall rates of acute dialysis and differences between the groups may not be large enough to detect during our follow-up time frame. Acute dialysis was a secondary outcome and the current study was not powered to detect such differences. Alternately, there may not actually be a significant impact of performing PN regarding this outcome. Processes involved in the development of AKI requiring brief episodes of dialysis may be distinct from those involved in the development of CKD and subsequent progression to ESRD. Once again, the associations between $\mathrm{AKI}$ and the subsequent development of CKD have been well established in the medical literature, but are yet to be defined in the surgical patient. 


\subsubsection{Overall survival}

The role of PN compared to $\mathrm{RN}$ in predicting overall survival was assessed as a secondary outcome. Our findings demonstrate a beneficial impact with improved overall survival in those undergoing PN compared to those who underwent RN. These findings persisted despite controlling for various demographic variables and patient comorbidity in a multivariable time to event model (HR 0.43, 0.37-0.51), as well as after utilizing propensity score matching (HR 0.45, 0.38-0.52).

Outcomes of overall survival remain one of the more controversial topics in this debate, and our findings are consistent with the majority of previously published retrospective series. One potential shortcoming of these findings is the inability to determine and control for disease stage in our cohort. Patients undergoing RN will, in general, present with much worse disease resulting in increased cancer-specific mortality as well as morbidity related to additional disease burden and treatments. ${ }^{153}$ Though it would be unlikely to see a reversal in trend given the strength of our hazard ratio, the effect may be dampened if analysis was isolated only to T1a tumors.

\subsubsection{Controversy regarding the EORTC clinical trial}

A major source of debate in this field revolves around the discrepancy between cumulative findings from the retrospective data compared to findings from the recent EORTC randomized clinical trial ${ }^{66}$ by van Poppel et al. comparing outcomes after RN and PN. The majority of retrospective series have demonstrated an independent benefit to PN compared to RN across various outcomes including renal function, cardiac morbidity, 
and overall survival. ${ }^{2,3,29,31,79-81}$ In contrast, the EORTC trial demonstrated a significant benefit to $\mathrm{RN}$ compared to $\mathrm{PN}$ in overall survival (81.1\% vs. $75.7 \%$ 10-year overall survival rate) amongst their 541 patients randomized and followed for a median of 9.3 years.

Although such discrepancies remain an important issue, it is important to differentiate the objectives of the current study and stress that our findings do not directly address these issues. We have chosen to assess the primary outcome of ESRD requiring RRT with the goal of delineating outcomes related to renal function. These specific outcomes were not reported or specifically addressed in the EORTC trial. Moreover, only a limited number of patients in the trial presented with abnormal renal function (normal serum Creatinine in $92 \%$ of patients), limiting their ability to assess the impact of surgical approach on renal functional outcomes across a broad spectrum of risk strata. Our population-level analysis offers a different perspective than that offered by the carefully selected cohort involved in the randomized trial.

On the other hand, the EORTC data do represent the only randomized trial data currently available to address this question. Despite attempts to adjust for baseline differences in retrospective studies, such attempts cannot completely account for selection bias between the surgical treatment groups. The only way to adequately answer this question would be another randomized trial, though such a study would be unlikely to happen. In the current setting, few physicians and patients are willing to randomize surgical management of small renal tumours.

The design of future trials addressing this question should consider the inclusion of, and better characterization of, patients with increasing comorbidity. The impact of 
surgical approach on the outcome of CKD among patients with HTN, DM, cardiovascular disease, and various levels of CKD will be important to individually assess. Also important in the design of future trials would be the inclusion of an objective measure of anatomic tumour characteristics. Such anatomic factors impact the difficulty of the surgery and thus subsequent patient outcomes. Several methods have been proposed and validated to standardize renal tumour anatomy, most notably the R.E.N.AL. Nephrometry Score. ${ }^{74}$

\subsection{Findings (General trends)}

\subsubsection{Utilization of PN}

One of the advantages of this large, province-wide cohort is the ability to assess current population-level practice patterns. We update the current trends in uptake of PN and demonstrate increasing utilization of this procedure through the year 2010 with an overall PN rate of 33\%. This represents the most updated data regarding uptake of PN and provides encouraging news for a procedure that has historically demonstrated a delayed and slow dissemination. ${ }^{84,86,135}$

We also further delineate important factors that may be impacting the selection process for surgical treatment. Patients undergoing PN compared to RN had higher rates of DM (21.6\% vs. $18.4 \%)$, HTN ( $42.6 \%$ vs. $41.3 \%)$, and pre-operative CKD ( $8.5 \%$ vs. 4.2\%). These comorbidities represent the most important known risk factors for the subsequent development of ESRD requiring $\mathrm{RRT}^{154}$ and are therefore important considerations in determining surgical treatment. The greater prevalence of comorbidity among patients undergoing PN compared to those undergoing $\mathrm{RN}$ demonstrates 
encouraging findings. As a medical profession, we may be making more appropriate decisions in the most recent years in selecting surgical treatment for our patients.

\subsubsection{Predictors of adverse outcome}

Many of the identified risk factors for adverse outcomes in this population are consistent with previously established risk factors. This held true for the majority of our outcomes but were particularly relevant in the analysis of our primary outcome of ESRD requiring RRT. Increasing age was associated with increasing rates of ESRD in a stepwise fashion with the exception of the oldest age group $(\geq 80)$. This may be expected given the competing risks associated with this elderly subset of the cohort. This may also be a reflection of selection bias in the most elderly patients, in that only the fittest are considered for any type of renal surgery. In addition, increasing measures of comorbidity were associated with a greater likelihood of developing ESRD. This stepwise relationship was evident regardless of the method of measuring comorbidity (Charlson comorbidity score or ADG score). Increasing incidence of ESRD was also associated with the presence of each of the individually identified ESRD risk factors (CKD, prior MI, DM, HTN). These are important findings to support both the face validity of the data and also to confirm prior established risk factors within our cohort.

\subsection{Limitations}

\subsubsection{Pathologic and staging data}

One of the important limitations of our study is the lack of pathologic and staging data. There is no ability to stratify the cohort based on tumour size or to control for 
tumour burden. However, information is available to identify patients who were diagnosed with metastatic disease during their initial presentation. These patients were then excluded from the analysis in order to limit the cohort to individuals with localized disease. Capture of such detail from these databases will likely have a high specificity, but the sensitivity of such diagnoses is uncertain. The impact of this may be minimal, given that the rates of patients initially presenting with metastatic disease and undergoing surgery is low (6\%) during the analyzed time period and has demonstrated a steady decline over the years in based on North American population studies. ${ }^{16,155}$

Regardless, patients initially presenting with $\mathrm{T} 1 \mathrm{a}(\leq 4 \mathrm{~cm})$ tumours represent an important subpopulation in which it is considered a standard of care to perform PN rather than $\mathrm{RN} .^{43}$ The major limitation of our pathologic data is the inability to isolate this population. This shortcoming, however, should not detract from our primary conclusions. Outcomes related to the development of ESRD or worsening renal function will not, in general, be impacted by initial tumour stage. Instead, such outcomes are dependent on patient comorbidity and surgical treatment, which are otherwise well captured in this study.

However, we would expect this to impact the results assessing outcomes of overall survival. Not limiting the cohort to patients with T1a tumours would bias findings towards worse overall survival in those undergoing $\mathrm{RN}$ since the population would be enriched with high-risk RN patients with a greater likelihood of succumbing to the disease or disease-related treatments (i.e. systemic therapy, additional surgery). Thus, the ability to account for tumor size and stage would likely attenuate current findings demonstrating a benefit of $\mathrm{PN}$ in improving overall survival. 


\subsubsection{Serum Creatinine levels}

Another major limitation of this study is the absence of laboratory values in our databases. Serum creatinine levels and estimated glomerular filtration rate (eGFR) represent our most objective (among those widely used in clinical practice) methods of assessing renal function. Unfortunately, we were unable to assess these specific values, but instead relied on an algorithm utilizing inpatient and outpatient diagnoses of CKD. We lack a true objective measure to capture this specific comorbidity in both the preoperative and post-operative setting, and we also lack the ability to capture and differentiate between various stages of CKD. This limitation should bias the results toward an underestimation of the actual rates of $\mathrm{CKD}$, as it fails to capture subclinical cases of renal dysfunction. Patients with low levels of renal dysfunction reflected only as mild increases in serum creatinine (or mild decreases in eGFR) and who have not attained an actual diagnosis of CKD will not be captured in this study. Secondly, we lose the ability to discriminate between various levels of CKD, which are often defined by distinct eGFR levels. A final concern related to the lack of serum creatinine levels is the potential discrepancy and inconsistency in coding for CKD diagnoses. No strict definitions were in place to determine who will or will not obtain such a diagnosis in either the inpatient or outpatient setting. Therefore, providers used variable criteria to assign patients a diagnosis of CKD.

Rates of patients developing all levels of renal dysfunction would intuitively follow similar patterns to those obtaining a clinical diagnosis of CKD. Therefore, the lack of serum creatinine levels would likely blunt any significant findings regarding the 
development of CKD. Furthermore, a clinical diagnosis of CKD represents an important, clinically relevant outcome and a unique method of identifying patients who develop CKD. Bias in this under-representation should not lean in a specific direction. Follow-up and monitoring for both of these patient groups should be similar given that all patients were surgically treated for RCC. Although the lack of serum creatinine levels presents challenges in the interpretation of results, it should not significantly impact current findings.

\subsubsection{Accuracy of capture of confounders}

With the use of large administrative databases not created for the specific purpose of research, one of the consistent limitations is the accuracy of capture of variables and outcomes. Less of an issue is "hard" outcomes and procedures, including the main effector (type of surgery) and the primary outcome (chronic dialysis or transplantation) in our study. Such variables are more reliably captured and distinctly coded because of the nature of the variable. Both of these variables have demonstrated good validity when compared to the medical record. ${ }^{102,103,106,156}$ A possible discrepancy regarding surgical coding involves conversion of procedure, typically from PN to RN. Identifying converted procedures would offer more insight into the intended procedures as opposed to the actual procedure performed. It is unclear whether surgical procedures were coded based on the planned operative procedure or the actual procedure performed. The direction of bias in the results will depend on how such procedures were coded. Regardless, such discrepancies will likely have little impact on the overall findings given the small number of patients undergoing unplanned conversion. Internal review of our institutional data 
comparing $\mathrm{CCP} / \mathrm{CCI}$ codes to abstracted pathology reports demonstrated greater than 99\% accuracy. Another possible discrepancy includes the coding of ablative approaches prior to OHIP developing dedicated billing codes for these procedures, however, very few of these cases would have pathologic confirmation, thus eliminating them from our cohort. Additionally, not including these patients does not impact on our findings, since they did not undergo either PN or RN.

Another concern in this regard is in the capture of comorbidities. Charlson comorbidity score and Johns Hopkin's ADG score are composite comorbidity scores which have been well validated across various populations and outcomes. They have not, however, been specifically validated in populations of patients undergoing surgical treatment for RCC. These comorbidity indexes have been utilized for this population to assess outcomes of overall mortality and peri-operative morbidity. ${ }^{112,113} \mathrm{~A}$ limitation of these composite scores is their ability to discriminate between specific comorbid diagnoses of particular interest. They do not offer the ability to specifically analyze and control for the most relevant risk factors including DM, HTN, cardiovascular disease, and pre-operative CKD. However, in this study, we also examined individual comorbidities associated with a greater risk of CKD.

DM and HTN have well-validated algorithms for capture across various linked inpatient and outpatient databases. ${ }^{118,119}$ Several algorithms have been utilized for the capture of CKD, though previously published algorithms utilize only a single database (outpatient or inpatient). ${ }^{127,128,157,158}$ A combination of these algorithms was utilized to incorporate capture of this diagnosis from either an outpatient or inpatient source in this 
study, thus increasing the sensitivity. This combined algorithm of capture has not, however, been previously validated or utilized in the literature.

Patients with acute kidney injury (AKI) were identified by the presence of a single billing code for acute dialysis. Other algorithms have been utilized in the literature, which we were unaware of at the time of analysis. Such methods identified AKI as the presence of an inpatient diagnostic code for AKI and an acute dialysis code associated with the same hospitalization. Utilization of this alternative algorithm may impact the ascertainment of AKI events and is a consideration in future work. It does not, however, impact on our primary outcome, which is ESRD requiring chronic dialysis or kidney transplantation.

\subsubsection{Ability to control for confounding}

Another relevant issue and limitation of our dataset and methodology is the ability to control for confounding. The selection bias between treatment groups has always been a concern in observational studies addressing this question. It would not be possible to completely address bias between these groups in retrospective studies. However, we addressed this by utilizing multiple methods of capturing comorbidity and multiple analytic methods of controlling for these confounders. Regarding methods of identifying comorbidity, composite scores originally developed from inpatient (Charlson) and outpatient (ADG) settings were utilized. In addition, specific comorbidities were individually identified (see above). Each of these comorbidities was identified as independent risk factors for many of our outcomes, offering face validity to the findings. Multiple analytic methods of controlling for confounders were utilized, including 
multivariable Cox PH models, propensity score analysis, and competing risks analysis. The strength and consistency of our findings across these various methods (Table 4.11) offers credibility and confidence in these findings.

The potential selection bias regarding patient morbidity can feasibly occur in either direction. It may be argued that individuals with greater comorbidity, and thus a greater likelihood of developing CKD, are more likely to undergo PN in hopes of preserving renal function. On the other hand, elderly patients with greater morbidity may be more likely to undergo RN because of the perception of fewer peri-operative complications and shorter convalescence, particularly with laparoscopic approaches. Evidence has not clearly resolved this issue in either direction, and data actually indicate that there may not be significant differences. Abouassaly et al., from a population-level analysis, did not identify differences in rates of HTN and DM in those undergoing PN compared to RN. DM and HTN were not significant independent predictors of undergoing PN, though older age was a predictor of $\mathrm{RN} .{ }^{83}$ Baseline differences between surgical groups in the current study indicate that patients undergoing RN may be older, yet have lower rates of pre-existing CKD. Other measures of comorbidity (ADG score, Charlson comorbidity score) were similar between the groups.

\subsubsection{Analysis of modern cohort}

Another important limitation is related to our findings of benefit for PN regarding ESRD in the modern cohort. This was a post-hoc analysis and not part of the original planned analysis. Post-hoc analyses always raise concerns about false positive results, though in retrospect such a subgroup analysis addresses a critical and valid question in 
that analysis of the modern cohort provides a more accurate representation of current practices regarding both surgical technique and expertise, as well as patient selection.

Actual rates of patients undergoing PN in the modern time frame were markedly higher than those in the earlier period (Figure 4.1). Moreover, differences in the behavior of PN patients between earlier and later time periods were demonstrated in our study, indicating significant, inherent differences between PN patients in these treatment periods. CIF curves (Figure 4.11) comparing rates of ESRD stratified by patients undergoing RN compared to $\mathrm{PN}$ cross at approximately 4.5 years of post-operative follow-up, signifying a shift in the relative behavior and potentially indicating heterogeneous treatment groups. Patients undergoing PN in the earlier years of the cohort and earlier years of adoption of the procedure may also suffer from worse peri-operative morbidity. Such inflated morbidity may in turn inflate the harms of PN. Finally, findings were not restricted to a specific start year for the modern cohort (Table 4.6), reducing the likelihood that our finding is a chance or false positive one. Ideally, however, our finding requires validation in an independent dataset.

\subsubsection{Further analysis}

Another area of potential limitation involves further analysis in specific areas that may provide additional information. A consideration would be further testing of nonproportionality for the various outcomes. Log-log graph comparisons were performed to confirm the proportionality of the relationship between surgical approach and the primary outcome of ESRD, however these tests were not performed for the secondary outcomes. Another consideration would be to expand the competing risks analysis to include 
subdistribution hazard ratios. Only univariable models were utilized in the current analysis. Inclusion of a multivariable model would help assess the impact of other variables on the outcomes while accounting for competing risks. Such analysis should be considered in future work.

\subsection{Impact and Significance}

The most significant impact of this work is its contribution to understanding the relationship between surgical treatments for RCC and the development of ESRD requiring RRT. Prior work has been performed assessing the impact of surgery on measures of CKD such as serum creatinine and eGFR, but to our knowledge no study has adequately assessed its relationship to definitive outcomes of renal failure. Such outcomes better represent the clinical and economic impact of surgical choices and are a more significant source of both patient morbidity and health resource utilization.

Incidence rates of ESRD requiring RRT are lower than other assessed outcomes, but represent an essential and underappreciated piece of the story. We provide the first clear evidence of this relationship and lend further support to the importance of nephronsparing surgery in the appropriately selected patient.

This study also further contributes to our understanding of the impact of surgical approach across a broad population of patients. We confirm findings from prior retrospective studies regarding the adverse impact of $\mathrm{RN}$ on renal function, cardiac morbidity, and overall survival. Our findings are unique in that we are able to study this relationship in a cohort that represents the entire province, across all ages, and with extensive capture of patient comorbidity. Few others studies have offered such a 
comprehensive amount of patient data from a population-level cohort. Given this degree of patient detail, we are much better able to control for confounding factors and address issues of bias between the groups, which is one of the major limitations of prior retrospective studies. That being said, however, we do recognize that we cannot entirely eliminate selection biases and residual confounding with the approaches we have used.

\subsection{Future Steps}

While we have gained significant knowledge and insight regarding the role of partial and radical nephrectomy in the surgical management of renal tumours, persistent gaps in our knowledge still remain. One area needing further exploration is the ability to delineate subsets of patients who will benefit the most from NSS. Our ability to stratify patients and their risk of development of various adverse outcomes remains limited given our current knowledge.

Especially relevant may be the role of surgically-induced CKD among patients of variable medical risk strata or with various stages of medically-induced CKD. The relevance of the excess surgical insult to renal function conferred by RN compared to PN may depend on the patient's likelihood of continued renal functional decline based on their pre-existing medical profile and risk factors. Future work may involve formal subgroup analysis involving all relevant patient variables and risk factors to help delineate these relationships. In summary, it has become apparent that not all patients should be treated the same and that we should work towards a more individualized approach as we continue to gain knowledge. 
Another important area to explore is the role of non-surgical treatments (ablative therapies and active surveillance) in the management of RCC. These have been demonstrated as feasible and promising treatment strategies and will likely see an increasing role in the management of SRMs in coming years. ${ }^{39,45}$ Non-surgical treatments may offer the best profiles for balancing cancer control while avoiding adverse outcomes, particularly in more frail or comorbidly ill older adults. The landscape is rapidly changing and these management strategies will likely be an important part of treatment algorithms in the near future, thus emphasizing the importance of analyzing their impact.

A final important aspect of future work should ultimately include the dissemination of this new knowledge. Our work remains relevant in daily clinical practice and has the potential for an immediate impact on patient care. Such areas of knowledge translation may include integration into clinical practice guidelines and methods of education for surgeons as well as patients. Unfortunately, dissemination of surgical knowledge has been slow in the past but efforts should continue in this realm in parallel to other areas of the clinical research.

\subsection{Conclusions}

In this population-based study utilizing linked administrative databases in the province of Ontario, a significant benefit was demonstrated in performing PN compared to $\mathrm{RN}$ for the treatment of RCC across a series of outcomes. The primary outcome in the current study was the development of ESRD. Significant benefit for those undergoing PN was demonstrated within a modern cohort (2003-2010) across various methods of 
controlling for bias. This represents the first demonstration of the association between surgical approach and "hard" renal outcomes such as ESRD. Several secondary outcomes were also assessed with a benefit for PN identified for the prevention of new-onset CKD, decreased rates of MI, and improved overall survival. Such findings lend further evidence for the benefit of PN, particularly related to outcomes of renal function. These represent important findings, though significant work remains particularly regarding identifying the patient groups who would benefit most from NSS as well as assessing the role of surgically versus medically induced CKD. 


\section{Glossary of Abbreviations}

\begin{tabular}{ll} 
ACG & Johns Hopkins Adjusted Clinical Groups \\
ADG & Johns Hopkins' Aggregated Diagnosis Groups \\
CADG & Collapsed ADGs \\
CCI & Canadian Classification of Health Interventions \\
CCO & Cancer Care Ontario \\
CCP & Canadian Classification of Diagnostic, Therapeutic, and Surgical Procedures \\
CI & confidence interval \\
CIHI & Canadian Institute of Health Information \\
CKD & chronic kidney disease \\
DAD & Discharge Abstract Database \\
DM & diabetes mellitus \\
ESRD & end stage renal disease \\
HIF & hypoxia-inducing factor \\
HR & hazards ratio \\
HTN & hypertension \\
ICD-9 & International Classification of Diseases-9 \\
ICD-10 & International Classification of Diseases-10 \\
ICES & Institute for Clinical Evaluative Sciences \\
MI & myocardial infarction \\
n/a & not applicable \\
NOS & not otherwise specified \\
OCR & Ontario Cancer Registry \\
OHIP & Ontario Health Insurance Plan \\
PH & proportional hazards \\
PN & partial nephrectomy \\
RCC & renal cell carcinoma \\
RN & radical nephrectomy \\
SEER & Surveillance Epidemiology and End Results \\
SRM & small renal mass \\
VHL & Von Hippel-Lindau \\
& \\
\hline
\end{tabular}




\section{REFERENCES}

1. Fergany AF, Hafez KS, Novick AC. Long-term results of nephron sparing surgery for localized renal cell carcinoma: 10-year followup. J Urol 2000;163:442-5. 2. Huang WC, Levey AS, Serio AM, et al. Chronic kidney disease after nephrectomy in patients with renal cortical tumours: a retrospective cohort study. Lancet Oncol 2006;7:735-40.

3. Huang WC, Elkin EB, Levey AS, Jang TL, Russo P. Partial nephrectomy versus radical nephrectomy in patients with small renal tumors--is there a difference in mortality and cardiovascular outcomes? J Urol 2009;181:55-61; discussion -2.

4. Go AS, Chertow GM, Fan D, McCulloch CE, Hsu CY. Chronic kidney disease and the risks of death, cardiovascular events, and hospitalization. N Engl J Med 2004;351:1296-305.

5. Abouassaly R, Alibhai SM, Tomlinson G, Timilshina N, Finelli A. Unintended consequences of laparoscopic surgery on partial nephrectomy for kidney cancer. J Urol 2009;183:467-72.

6. Ferlay J, Shin HR, Bray F, Forman D, Mathers C, Parkin DM. Estimates of worldwide burden of cancer in 2008: GLOBOCAN 2008. Int J Cancer 2011;127:2893917.

7. Pantuck AJ, Zisman A, Belldegrun AS. The changing natural history of renal cell carcinoma. J Urol 2001;166:1611-23.

8. Chow WH, Dong LM, Devesa SS. Epidemiology and risk factors for kidney cancer. Nat Rev Urol 2010;7:245-57.

9. Hunt JD, van der Hel OL, McMillan GP, Boffetta P, Brennan P. Renal cell carcinoma in relation to cigarette smoking: meta-analysis of 24 studies. Int J Cancer 2005;114:101-8.

10. Adams KF, Leitzmann MF, Albanes D, et al. Body size and renal cell cancer incidence in a large US cohort study. Am J Epidemiol 2008;168:268-77.

11. Bonsib SM. Renal cystic diseases and renal neoplasms: a mini-review. Clin J Am Soc Nephrol 2009;4:1998-2007.

12. Ljungberg B, Campbell SC, Choi HY, et al. The epidemiology of renal cell carcinoma. Eur Urol 2011;60:615-21.

13. Miller DC, Ruterbusch J, Colt JS, et al. Contemporary clinical epidemiology of renal cell carcinoma: insight from a population based case-control study. J Urol 2010;184:2254-8.

14. Chow WH, Devesa SS, Warren JL, Fraumeni JF, Jr. Rising incidence of renal cell cancer in the United States. JAMA 1999;281:1628-31.

15. Hollingsworth JM, Miller DC, Daignault S, Hollenbeck BK. Rising incidence of small renal masses: a need to reassess treatment effect. J Natl Cancer Inst 2006;98:1331-4.

16. Kane CJ, Mallin K, Ritchey J, Cooperberg MR, Carroll PR. Renal cell cancer stage migration: analysis of the National Cancer Data Base. Cancer 2008;113:78-83. 17. Robson CJ, Churchill BM, Anderson W. The results of radical nephrectomy for renal cell carcinoma. J Urol 1969;101:297-301. 
18. Tsui KH, Shvarts O, Barbaric Z, Figlin R, de Kernion JB, Belldegrun A. Is adrenalectomy a necessary component of radical nephrectomy? UCLA experience with 511 radical nephrectomies. J Urol 2000;163:437-41.

19. Kuczyk M, Munch T, Machtens S, et al. The need for routine adrenalectomy during surgical treatment for renal cell cancer: the Hannover experience. BJU Int 2002;89:517-22.

20. von Knobloch R, Seseke F, Riedmiller H, Grone HJ, Walthers EM, Kalble T. Radical nephrectomy for renal cell carcinoma: Is adrenalectomy necessary? Eur Urol 1999;36:303-8.

21. Gill IS, McClennan BL, Kerbl K, Carbone JM, Wick M, Clayman RV. Adrenal involvement from renal cell carcinoma: predictive value of computerized tomography. J Urol 1994;152:1082-5.

22. Portis AJ, Yan Y, Landman J, et al. Long-term followup after laparoscopic radical nephrectomy. J Urol 2002;167:1257-62.

23. Permpongkosol S, Chan DY, Link RE, et al. Long-term survival analysis after laparoscopic radical nephrectomy. J Urol 2005;174:1222-5.

24. Jeong W, Rha $\mathrm{KH}, \mathrm{Kim} \mathrm{HH}$, et al. Comparison of laparoscopic radical nephrectomy and open radical nephrectomy for pathologic stage T1 and T2 renal cell carcinoma with clear cell histologic features: a multi-institutional study. Urology 2010;77:819-24.

25. Permpongkosol S, Chan DY, Link RE, Jarrett TW, Kavoussi LR. Laparoscopic radical nephrectomy: long-term outcomes. J Endourol 2005;19:628-33.

26. Van Poppel H, Da Pozzo L, Albrecht W, et al. A prospective randomized EORTC intergroup phase 3 study comparing the complications of elective nephronsparing surgery and radical nephrectomy for low-stage renal cell carcinoma. Eur Urol 2007;51:1606-15.

27. Herrlinger A, Schrott KM, Schott G, Sigel A. What are the benefits of extended dissection of the regional renal lymph nodes in the therapy of renal cell carcinoma. J Urol 1991;146:1224-7.

28. Provet J, Tessler A, Brown J, Golimbu M, Bosniak M, Morales P. Partial nephrectomy for renal cell carcinoma: indications, results and implications. J Urol 1991;145:472-6.

29. Lerner SE, Hawkins CA, Blute ML, et al. Disease outcome in patients with low stage renal cell carcinoma treated with nephron sparing or radical surgery. J Urol 1996;155:1868-73.

30. Herr HW. Partial nephrectomy for incidental renal cell carcinoma. Br J Urol 1994;74:431-3.

31. Butler BP, Novick AC, Miller DP, Campbell SA, Licht MR. Management of small unilateral renal cell carcinomas: radical versus nephron-sparing surgery. Urology 1995;45:34-40; discussion -1.

32. Lee CT, Katz J, Shi W, Thaler HT, Reuter VE, Russo P. Surgical management of renal tumors $4 \mathrm{~cm}$. or less in a contemporary cohort. J Urol 2000;163:730-6.

33. Crepel M, Jeldres C, Sun M, et al. A population-based comparison of cancercontrol rates between radical and partial nephrectomy for T1A renal cell carcinoma. Urology 2010;76:883-8. 
34. Gill IS, Kavoussi LR, Lane BR, et al. Comparison of 1,800 laparoscopic and open partial nephrectomies for single renal tumors. J Urol 2007;178:41-6.

35. Lane BR, Gill IS. 7-year oncological outcomes after laparoscopic and open partial nephrectomy. J Urol;183:473-9.

36. Thompson RH, Siddiqui S, Lohse CM, Leibovich BC, Russo P, Blute ML. Partial versus radical nephrectomy for 4 to $7 \mathrm{~cm}$ renal cortical tumors. J Urol 2009;182:2601-6.

37. Aron M, Gill IS. Minimally invasive nephron-sparing surgery (MINSS) for renal tumours. Part II: probe ablative therapy. Eur Urol 2007;51:348-57.

38. Stern JM, Gupta A, Raman JD, et al. Radiofrequency ablation of small renal cortical tumours in healthy adults: renal function preservation and intermediate oncological outcome. BJU Int 2009;104:786-9.

39. Tracy CR, Raman JD, Donnally C, Trimmer CK, Cadeddu JA. Durable oncologic outcomes after radiofrequency ablation: experience from treating 243 small renal masses over 7.5 years. Cancer 2010;116:3135-42.

40. Erdeljan P, Dhar M, Wignall G, Kozak R, Pautler SE. Thermal ablation of small renal masses: intermediate outcomes from a Canadian center. Can J Urol 2011;18:5903-7.

41. Johnson DB, Solomon SB, Su LM, et al. Defining the complications of cryoablation and radio frequency ablation of small renal tumors: a multiinstitutional review. J Urol 2004;172:874-7.

42. Laguna MP, Beemster P, Kumar V, et al. Perioperative morbidity of laparoscopic cryoablation of small renal masses with ultrathin probes: a European multicentre experience. Eur Urol 2009;56:355-61.

43. Campbell SC, Novick AC, Belldegrun A, et al. Guideline for management of the clinical T1 renal mass. J Urol 2009;182:1271-9.

44. Van Poppel H, Becker F, Cadeddu JA, et al. Treatment of localised renal cell carcinoma. Eur Urol 2011;60:662-72.

45. Jewett MA, Mattar K, Basiuk J, et al. Active surveillance of small renal masses: progression patterns of early stage kidney cancer. Eur Urol 2011;60:39-44.

46. Leveridge MJ, Finelli A, Kachura JR, et al. Outcomes of small renal mass needle core biopsy, nondiagnostic percutaneous biopsy, and the role of repeat biopsy. Eur Urol 2011;60:578-84.

47. Yap SA, Stakhovskyi 0, Finelli A. The emerging role of percutaneous biopsy in diagnosis and management of small renal masses. Curr Opin Urol 2012;22:360-4.

48. Motzer RJ, Rini BI, Bukowski RM, et al. Sunitinib in patients with metastatic renal cell carcinoma. JAMA 2006;295:2516-24.

49. Motzer RJ, Hutson TE, Tomczak P, et al. Sunitinib versus interferon alfa in metastatic renal-cell carcinoma. N Engl J Med 2007;356:115-24.

50. Ratain MJ, Eisen T, Stadler WM, et al. Phase II placebo-controlled randomized discontinuation trial of sorafenib in patients with metastatic renal cell carcinoma. J Clin Oncol 2006;24:2505-12.

51. Yang JC, Haworth L, Sherry RM, et al. A randomized trial of bevacizumab, an anti-vascular endothelial growth factor antibody, for metastatic renal cancer. N Engl J Med 2003;349:427-34. 
52. Hudes G, Carducci M, Tomczak P, et al. Temsirolimus, interferon alfa, or both for advanced renal-cell carcinoma. N Engl J Med 2007;356:2271-81.

53. Flanigan RC, Salmon SE, Blumenstein BA, et al. Nephrectomy followed by interferon alfa- $2 \mathrm{~b}$ compared with interferon alfa- $2 \mathrm{~b}$ alone for metastatic renal-cell cancer. N Engl J Med 2001;345:1655-9.

54. Mickisch GH, Garin A, van Poppel H, de Prijck L, Sylvester R. Radical nephrectomy plus interferon-alfa-based immunotherapy compared with interferon alfa alone in metastatic renal-cell carcinoma: a randomised trial. Lancet 2001;358:966-70.

55. D'Armiento M, Damiano R, Feleppa B, Perdona S, Oriani G, De Sio M. Elective conservative surgery for renal carcinoma versus radical nephrectomy: a prospective study. Br J Urol 1997;79:15-9.

56. Ljungberg B, Cowan NC, Hanbury DC, et al. EAU guidelines on renal cell carcinoma: the 2010 update. Eur Urol 2010;58:398-406.

57. Jewett M, Finelli A, Kollmannsberger C, et al. Management of kidney cancer: canadian kidney cancer forum consensus update 2011. Can Urol Assoc J 2012;6:1622.

58. Dulabon LM, Lowrance WT, Russo P, Huang WC. Trends in renal tumor surgery delivery within the United States. Cancer 2010;116:2316-21.

59. Marszalek M, Carini M, Chlosta P, et al. Positive surgical margins after nephron-sparing surgery. Eur Urol 2011;61:757-63.

60. Breda A, Stepanian SV, Liao J, et al. Positive margins in laparoscopic partial nephrectomy in 855 cases: a multi-institutional survey from the United States and Europe. J Urol 2007;178:47-50; discussion

61. Yossepowitch 0 , Thompson RH, Leibovich BC, et al. Positive surgical margins at partial nephrectomy: predictors and oncological outcomes. J Urol 2008;179:215863.

62. Permpongkosol S, Colombo JR, Jr., Gill IS, Kavoussi LR. Positive surgical parenchymal margin after laparoscopic partial nephrectomy for renal cell carcinoma: oncological outcomes. J Urol 2006;176:2401-4.

63. Stephenson AJ, Hakimi AA, Snyder ME, Russo P. Complications of radical and partial nephrectomy in a large contemporary cohort. J Urol 2004;171:130-4.

64. Stephenson AJ, Chetner MP, Rourke K, et al. Guidelines for the surveillance of localized renal cell carcinoma based on the patterns of relapse after nephrectomy. J Urol 2004;172:58-62.

65. Hafez KS, Fergany AF, Novick AC. Nephron sparing surgery for localized renal cell carcinoma: impact of tumor size on patient survival, tumor recurrence and TNM staging. J Urol 1999;162:1930-3.

66. Van Poppel H, Da Pozzo L, Albrecht W, et al. A prospective, randomised EORTC intergroup phase 3 study comparing the oncologic outcome of elective nephron-sparing surgery and radical nephrectomy for low-stage renal cell carcinoma. Eur Urol 2010;59:543-52.

67. Lau WK, Blute ML, Weaver AL, Torres VE, Zincke H. Matched comparison of radical nephrectomy vs nephron-sparing surgery in patients with unilateral renal cell carcinoma and a normal contralateral kidney. Mayo Clin Proc 2000;75:1236-42. 
68. Licht MR, Novick AC, Goormastic M. Nephron sparing surgery in incidental versus suspected renal cell carcinoma. J Urol 1994;152:39-42.

69. Patard JJ, Shvarts 0, Lam JS, et al. Safety and efficacy of partial nephrectomy for all T1 tumors based on an international multicenter experience. J Urol 2004;171:2181-5, quiz 435.

70. Joudi FN, Allareddy V, Kane CJ, Konety BR. Analysis of complications following partial and total nephrectomy for renal cancer in a population based sample. J Urol 2007;177:1709-14.

71. Abouassaly R, Alibhai SM, Tomlinson GA, Urbach DR, Finelli A. The effect of age on the morbidity of kidney surgery. J Urol 2011;186:811-6.

72. Corman JM, Penson DF, Hur K, et al. Comparison of complications after radical and partial nephrectomy: results from the National Veterans Administration Surgical Quality Improvement Program. BJU Int 2000;86:782-9.

73. Ficarra V, Novara G, Secco S, et al. Preoperative aspects and dimensions used for an anatomical (PADUA) classification of renal tumours in patients who are candidates for nephron-sparing surgery. Eur Urol 2009;56:786-93.

74. Kutikov A, Uzzo RG. The R.E.N.A.L. nephrometry score: a comprehensive standardized system for quantitating renal tumor size, location and depth. J Urol 2009;182:844-53.

75. Simmons MN, Ching CB, Samplaski MK, Park CH, Gill IS. Kidney tumor location measurement using the $\mathrm{C}$ index method. J Urol 2010;183:1708-13.

76. Bruner B, Breau RH, Lohse CM, Leibovich BC, Blute ML. Renal nephrometry score is associated with urine leak after partial nephrectomy. BJU Int 2010;108:6772 .

77. McKiernan J, Simmons R, Katz J, Russo P. Natural history of chronic renal insufficiency after partial and radical nephrectomy. Urology 2002;59:816-20.

78. Klarenbach S, Moore RB, Chapman DW, Dong J, Braam B. Adverse renal outcomes in subjects undergoing nephrectomy for renal tumors: a population-based analysis. Eur Urol 2010;59:333-9.

79. Tan HJ, Norton EC, Ye Z, Hafez KS, Gore JL, Miller DC. Long-term survival following partial vs radical nephrectomy among older patients with early-stage kidney cancer. JAMA 2012;307:1629-35.

80. Thompson RH, Boorjian SA, Lohse CM, et al. Radical nephrectomy for pT1a renal masses may be associated with decreased overall survival compared with partial nephrectomy. J Urol 2008;179:468-71; discussion 72-3.

81. Zini L, Perrotte P, Capitanio U, et al. Radical versus partial nephrectomy: effect on overall and noncancer mortality. Cancer 2009;115:1465-71.

82. Patel SG, Penson DF, Pabla B, et al. National trends in the use of partial nephrectomy: a rising tide that has not lifted all boats. J Urol 2012;187:816-21. 83. Abouassaly R, Finelli A, Tomlinson GA, Urbach DR, Alibhai SM. How often are patients with diabetes or hypertension being treated with partial nephrectomy for renal cell carcinoma? A population-based analysis. BJU Int 2011;108:1806-12. 84. Miller DC, Daignault S, Wolf JS, Jr., et al. Hospital characteristics and use of innovative surgical therapies among patients with kidney cancer. Med Care 2008;46:372-9. 
85. Porter MP, Lin DW. Trends in renal cancer surgery and patient provider characteristics associated with partial nephrectomy in the United States. Urol Oncol 2007;25:298-302.

86. Miller DC, Saigal CS, Banerjee M, Hanley J, Litwin MS. Diffusion of surgical innovation among patients with kidney cancer. Cancer 2008;112:1708-17.

87. Abouassaly R, Alibhai SM, Tomlinson GA, Urbach DR, Finelli A. The effect of age on the morbidity of kidney surgery. J Urol;186:811-6.

88. Collins AJ, Foley RN, Gilbertson DT, Chen SC. The state of chronic kidney disease, ESRD, and morbidity and mortality in the first year of dialysis. Clin J Am Soc Nephrol 2009;4 Suppl 1:S5-11.

89. Collins AJ, Chen SC, Gilbertson DT, Foley RN. CKD surveillance using administrative data: impact on the health care system. Am J Kidney Dis 2009;53:S27-36.

90. Lee H, Manns B, Taub K, et al. Cost analysis of ongoing care of patients with end-stage renal disease: the impact of dialysis modality and dialysis access. Am J Kidney Dis 2002;40:611-22.

91. Cancer Care Ontario. https://www.cancercare.on.ca (2012).

92. Holowaty E, Moravan V, Lee G. A reabstraction study to estimate the completeness and accuracy of data elements in the Ontario Cancer Registry. Report to Health Canada 1996.

93. Institute of Clinical and Evaluative Sciences. http://www.ices.on.ca/ (2012).

94. Canadian Institute for Health Information (CIHI). http://www.cihi.ca. (2012).

95. Canadian Institute for Health Information: CIHI data quality study of the 2005-2006 Discharge

Abstract Database.

96. Canadian Institute for Health Information: CIHI data quality study of the 2006-2007 Discharge

Abstract Database.

97. Canadian Institute for Health Information: Data quality of the Discharge Abstract Database following the first-year implementation of the ICD-10-CA/CCI Final Report.

98. Canadian Institute for Health Information: Discharge Abstract Database data quality re-abstraction study: Combined findings for fiscal years 1999/2000 and $2000 / 2001$.

99. Ontario Health Insurance Plan.

http://www.health.gov.on.ca/en/public/programs/ohip (2012).

100. Keegan KA, Schupp CW, Chamie K, Hellenthal NJ, Evans CP, Koppie TM.

Histopathology of surgically treated renal cell carcinoma: survival differences by subtype and stage. J Urol 2012;188:391-7.

101. Williams J, Young W. Inventory studies on the accuracy of Canadian Health Administrative

Databases. 1996.

102. Hawker GA, Coyte PC, Wright JG, Paul JE, Bombardier C. Accuracy of administrative data for assessing outcomes after knee replacement surgery. J Clin Epidemiol 1997;50:265-73. 
103. Alibhai S. Do age and comorbidity influence the treatment of localized prostate cancer? DeptHealth Admin 2001;169.

104. Quinn RR, Laupacis A, Austin PC, et al. Using administrative datasets to study outcomes in dialysis patients: a validation study. Med Care;48:745-50.

105. Liangos O, Wald R, O'Bell JW, Price L, Pereira BJ, Jaber BL. Epidemiology and outcomes of acute renal failure in hospitalized patients: a national survey. Clin J Am Soc Nephrol 2006;1:43-51.

106. Alibhai SM, Leach M, Tomlinson G, et al. 30-day mortality and major complications after radical prostatectomy: influence of age and comorbidity. J Natl Cancer Inst 2005;97:1525-32.

107. Deyo RA, Cherkin DC, Ciol MA. Adapting a clinical comorbidity index for use with ICD-9-CM administrative databases. J Clin Epidemiol 1992;45:613-9.

108. Charlson ME, Pompei P, Ales KL, MacKenzie CR. A new method of classifying prognostic comorbidity in longitudinal studies: development and validation. J Chronic Dis 1987;40:373-83.

109. Sundararajan V, Quan H, Halfon P, et al. Cross-national comparative performance of three versions of the ICD-10 Charlson index. Med Care 2007;45:1210-5.

110. Sundararajan V, Henderson T, Perry C, Muggivan A, Quan H, Ghali WA. New ICD-10 version of the Charlson comorbidity index predicted in-hospital mortality. J Clin Epidemiol 2004;57:1288-94.

111. Rochon PA, Katz JN, Morrow LA, et al. Comorbid illness is associated with survival and length of hospital stay in patients with chronic disability. A prospective comparison of three comorbidity indices. Med Care 1996;34:1093-101.

112. Kutikov A, Egleston BL, Canter D, Smaldone MC, Wong YN, Uzzo RG. Competing risks of death in patients with localized renal cell carcinoma: a comorbidity based model. J Urol 2012;188:2077-83.

113. Vricella GJ, Finelli A, Alibhai SM, Ponsky LE, Abouassaly R. The true risk of blood transfusion after nephrectomy for renal masses: a population-based study. BJU Int 2013.

114. Weiner J. The Johns Hopkins ACG System Reference Manual (Version 8.2). December 2008.

115. Austin PC, Walraven C. The mortality risk score and the ADG score: two points-based scoring systems for the Johns Hopkins aggregated diagnosis groups to predict mortality in a general adult population cohort in Ontario, Canada. Med Care 2011;49:940-7.

116. Petersen LA, Pietz K, Woodard LD, Byrne M. Comparison of the predictive validity of diagnosis-based risk adjusters for clinical outcomes. Med Care 2005;43:61-7.

117. Austin PC, van Walraven C, Wodchis WP, Newman A, Anderson GM. Using the Johns Hopkins Aggregated Diagnosis Groups (ADGs) to predict mortality in a general adult population cohort in Ontario, Canada. Med Care 2011;49:932-9. 118. Hux JE, Ivis F, Flintoft V, Bica A. Diabetes in Ontario: determination of prevalence and incidence using a validated administrative data algorithm. Diabetes Care 2002;25:512-6. 
119. Tu K, Campbell NR, Chen ZL, Cauch-Dudek KJ, McAlister FA. Accuracy of administrative databases in identifying patients with hypertension. Open Med 2007;1:e18-26.

120. Vermeulen MJ, Tu JV, Schull MJ. ICD-10 adaptations of the Ontario acute myocardial infarction mortality prediction rules performed as well as the original versions. J Clin Epidemiol 2007;60:971-4.

121. Tu JV, Austin PC, Walld R, Roos L, Agras J, McDonald KM. Development and validation of the Ontario acute myocardial infarction mortality prediction rules. J Am Coll Cardiol 2001;37:992-7.

122. Tu JV, Naylor CD, Austin P. Temporal changes in the outcomes of acute myocardial infarction in Ontario, 1992-1996. CMAJ 1999;161:1257-61.

123. Austin PC, Daly PA, Tu JV. A multicenter study of the coding accuracy of hospital discharge administrative data for patients admitted to cardiac care units in Ontario. Am Heart J 2002;144:290-6.

124. Alter DA, Naylor CD, Austin PC, Tu JV. Long-term MI outcomes at hospitals with or without on-site revascularization. JAMA 2001;285:2101-8.

125. Gislason GH, Jacobsen S, Rasmussen JN, et al. Risk of death or reinfarction associated with the use of selective cyclooxygenase- 2 inhibitors and nonselective nonsteroidal antiinflammatory drugs after acute myocardial infarction. Circulation 2006;113:2906-13.

126. Vlasschaert ME, Bejaimal SA, Hackam DG, et al. Validity of administrative database coding for kidney disease: a systematic review. Am J Kidney Dis;57:29-43. 127. Lee DS, Donovan L, Austin PC, et al. Comparison of coding of heart failure and comorbidities in administrative and clinical data for use in outcomes research. Med Care 2005;43:182-8.

128. Ontario Hospital Association, Ontario Ministry of Health and Hospital Medical Records Institute: Report of the Ontario data quality reabstracting study. 1991.

129. Juurlink D, Preyra C, Croxford R, et al. Canadian Institute for Health Information DIascharge Abstract Database: A Validation Study. 2006.

130. Power and sample size calculation (Department of biostatistics, Vanderbilt University). http://biostatmcvanderbiltedu/wiki/Main/PowerSampleSize.

131. Feise RJ. Do multiple outcome measures require $p$-value adjustment? BMC Med Res Methodol 2002;2:8.

132. Stoltzfus JC. Logistic regression: a brief primer. Acad Emerg Med 2011;18:1099-104.

133. Glynn RJ, Schneeweiss S, Sturmer T. Indications for propensity scores and review of their use in pharmacoepidemiology. Basic Clin Pharmacol Toxicol 2006;98:253-9.

134. Pintile M. Competing Risks: A Practical Perspective: Wiley; 2006.

135. Miller DC, Hollingsworth JM, Hafez KS, Daignault S, Hollenbeck BK. Partial nephrectomy for small renal masses: an emerging quality of care concern? J Urol 2006;175:853-7; discussion 8.

136. Hollenbeck BK, Taub DA, Miller DC, Dunn RL, Wei JT. National utilization trends of partial nephrectomy for renal cell carcinoma: a case of underutilization? Urology 2006;67:254-9. 
137. Clark AT, Breau RH, Morash C, Fergusson D, Doucette S, Cagiannos I.

Preservation of renal function following partial or radical nephrectomy using 24hour creatinine clearance. Eur Urol 2008;54:143-49.

138. Turin TC, Tonelli M, Manns BJ, et al. Lifetime risk of ESRD. J Am Soc Nephrol 2012;23:1569-78.

139. Barlow LJ, Korets R, Laudano M, Benson M, McKiernan J. Predicting renal functional outcomes after surgery for renal cortical tumours: a multifactorial analysis. BJU Int 2009;106:489-92.

140. Malcolm JB, Bagrodia A, Derweesh IH, et al. Comparison of rates and risk factors for developing chronic renal insufficiency, proteinuria and metabolic acidosis after radical or partial nephrectomy. BJU Int 2009;104:476-81.

141. Hallan SI, Matsushita K, Sang Y, et al. Age and Association of Kidney Measures With Mortality and End-stage Renal Disease. JAMA:1-12.

142. Turin TC, Tonelli M, Manns BJ, Ravani P, Ahmed SB, Hemmelgarn BR. Chronic kidney disease and life expectancy. Nephrol Dial Transplant;27:3182-6.

143. Hemmelgarn BR, Manns BJ, Lloyd A, et al. Relation between kidney function, proteinuria, and adverse outcomes. JAMA;303:423-9.

144. Mahmoodi BK, Matsushita K, Woodward M, et al. Associations of kidney disease measures with mortality and end-stage renal disease in individuals with and without hypertension: a meta-analysis. Lancet;380:1649-61.

145. Fox CS, Matsushita K, Woodward M, et al. Associations of kidney disease measures with mortality and end-stage renal disease in individuals with and without diabetes: a meta-analysis. Lancet;380:1662-73.

146. Fox CS, Matsushita K, Woodward M, et al. Associations of kidney disease measures with mortality and end-stage renal disease in individuals with and without diabetes: a meta-analysis. Lancet 2012;380:1662-73.

147. Mahmoodi BK, Matsushita K, Woodward M, et al. Associations of kidney disease measures with mortality and end-stage renal disease in individuals with and without hypertension: a meta-analysis. Lancet 2012;380:1649-61.

148. Lane BR, Campbell SC, Demirjian S, Fergany AF. Surgically-induced chronic kidney disease may be associated with lesser risk of progression and mortality than medical chronic kidney disease. Journal of Urololgy 2013;in press.

149. Miller DC, Schonlau M, Litwin MS, Lai J, Saigal CS. Renal and cardiovascular morbidity after partial or radical nephrectomy. Cancer 2008;112:511-20.

150. Coca SG, Singanamala S, Parikh CR. Chronic kidney disease after acute kidney injury: a systematic review and meta-analysis. Kidney Int 2011;81:442-8.

151. Goldberg R, Dennen P. Long-term outcomes of acute kidney injury. Adv Chronic Kidney Dis 2008;15:297-307.

152. Cho A, Lee JE, Kwon GY, et al. Post-operative acute kidney injury in patients with renal cell carcinoma is a potent risk factor for new-onset chronic kidney disease after radical nephrectomy. Nephrol Dial Transplant 2011;26:3496-501. 153. Thompson RH, Kaag M, Vickers A, et al. Contemporary use of partial nephrectomy at a tertiary care center in the United States. J Urol 2009;181:993-7. 154. Chang TI, Li S, Chen SC, et al. Risk factors for ESRD in individuals with preserved estimated GFR with and without albuminuria: results from the Kidney Early Evaluation Program (KEEP). Am J Kidney Dis 2013;61:S4-11. 
155. Thompson RH, Hill JR, Babayev Y, et al. Metastatic renal cell carcinoma risk according to tumor size. J Urol 2009;182:41-5.

156. Quinn RR, Laupacis A, Austin PC, et al. Using administrative datasets to study outcomes in dialysis patients: a validation study. Med Care 2010;48:745-50.

157. Vlasschaert ME, Bejaimal SA, Hackam DG, et al. Validity of administrative database coding for kidney disease: a systematic review. Am J Kidney Dis 2010;57:29-43.

158. Juurlink D, Preyra C, Croxford R. Canadian Institute for Health Information DIascharge Abstract Database: A Validation Study. 2006. 


\section{APPENDIX A}

Table 1. Distribution of demographic and clinical variables within each propensity score quintile

\begin{tabular}{|c|c|c|c|c|c|c|c|c|c|c|}
\hline & Quintile 1 & & Quintile 2 & & Quintile 3 & & Quintile 4 & & Quintile 5 & \\
\hline & $\mathrm{RN}$ & PN & $\mathrm{RN}$ & PN & $\mathrm{RN}$ & $\mathrm{PN}$ & $\mathrm{RN}$ & PN & $\mathrm{RN}$ & PN \\
\hline \multicolumn{11}{|l|}{ Sex } \\
\hline Female & $1122(48 \%)$ & $20(41 \%)$ & $747(35 \%)$ & $102(39 \%)$ & $968(50 \%)$ & $198(47 \%)$ & $541(30 \%)$ & $169(32 \%)$ & $552(34 \%)$ & $283(34 \%)$ \\
\hline Male & $1202(52 \%)$ & $29(59 \%)$ & $1410(65 \%)$ & $157(61 \%)$ & $971(50 \%)$ & $223(53 \%)$ & $1235(70 \%)$ & $365(68 \%)$ & $1082(66 \%)$ & $561(66 \%)$ \\
\hline \multicolumn{11}{|l|}{ Age group } \\
\hline$<50$ & $212(9 \%)$ & $3(6 \%)$ & $290(13 \%)$ & $24(9 \%)$ & $532(27 \%)$ & $114(27 \%)$ & $92(5 \%)$ & $29(5 \%)$ & $661(40 \%)$ & $370(44 \%)$ \\
\hline $50-59$ & $502(22 \%)$ & $17(35 \%)$ & $281(13 \%)$ & $34(13 \%)$ & $497(26 \%)$ & $99(24 \%)$ & $489(28 \%)$ & $144(27 \%)$ & $639(39 \%)$ & $298(35 \%)$ \\
\hline $60-69$ & $690(30 \%)$ & $17(35 \%)$ & $673(31 \%)$ & $95(36 \%)$ & $337(17 \%)$ & $88(21 \%)$ & $790(45 \%)$ & $220(41 \%)$ & $214(13 \%)$ & $119(14 \%)$ \\
\hline $70-79$ & $627(27 \%)$ & $7(14 \%)$ & $628(29 \%)$ & $78(30 \%)$ & $500(26 \%)$ & $105(25 \%)$ & $391(22 \%)$ & $138(26 \%)$ & $118(7 \%)$ & $55(7 \%)$ \\
\hline$\geq 80$ & $293(13 \%)$ & $5(10 \%)$ & $285(13 \%)$ & $28(11 \%)$ & $73(4 \%)$ & $15(4 \%)$ & $14(0.8 \%)$ & $3(0.6 \%)$ & $2(0.1 \%)$ & $2(0.2 \%)$ \\
\hline \multicolumn{11}{|l|}{ Year group } \\
\hline $1995-1999$ & $2188(94 \%)$ & $45(92 \%)$ & $393(18 \%)$ & $28(10 \%)$ & $1(0.1 \%)$ & $1(0.2 \%)$ & $5(0.3 \%)$ & $0(0 \%)$ & $2(0.1 \%)$ & $2(0.2 \%)$ \\
\hline $2000-2004$ & $127(5 \%)$ & $4(0.2 \%)$ & $1522(71 \%)$ & $206(80 \%)$ & $1163(60 \%)$ & $235(56 \%)$ & $119(7 \%)$ & $48(9 \%)$ & $41(2.5 \%)$ & $25(3 \%)$ \\
\hline $2005-2010$ & $9(0.4 \%)$ & $0(0 \%)$ & $242(11 \%)$ & $25(10 \%)$ & $775(40 \%)$ & $185(44 \%)$ & $1652(93 \%)$ & $486(91 \%)$ & $1591(97 \%)$ & $817(97 \%)$ \\
\hline \multicolumn{11}{|l|}{ Histology } \\
\hline Clear cell & $218(9 \%)$ & $5(10 \%)$ & $594(28 \%)$ & $74(29 \%)$ & $885(46 \%)$ & $202(48 \%)$ & $773(44 \%)$ & $232(43 \%)$ & $496(30 \%)$ & $244(29 \%)$ \\
\hline Papillary & $4(0.2 \%)$ & $0(0 \%)$ & $47(2 \%)$ & $10(4 \%)$ & $67(3 \%)$ & $17(4 \%)$ & $128(7 \%)$ & $32(6 \%)$ & $253(15 \%)$ & $158(19 \%)$ \\
\hline Chromophobe & $1(0.04 \%)$ & $0(0 \%)$ & $42(2 \%)$ & $10(4 \%)$ & $56(3 \%)$ & $6(1 \%)$ & $97(5 \%)$ & $32(6 \%)$ & $175(11 \%)$ & $83(10 \%)$ \\
\hline NOS & $2102(90 \%)$ & $44(90 \%)$ & $1474(68 \%)$ & $165(64 \%)$ & $931(48 \%)$ & $196(50 \%)$ & $778(44 \%)$ & $238(45 \%)$ & $710(43 \%)$ & $359(43 \%)$ \\
\hline \multicolumn{11}{|l|}{$\begin{array}{l}\text { Weighted ADG } \\
\text { (quintile) }\end{array}$} \\
\hline 1 & $428(18 \%)$ & $10(20 \%)$ & $357(17 \%)$ & $42(16 \%)$ & $452(23 \%)$ & $86(20 \%)$ & $320(18 \%)$ & $75(14 \%)$ & $364(22 \%)$ & $183(22 \%)$ \\
\hline 2 & $482(21 \%)$ & $11(22 \%)$ & $495(23 \%)$ & $57(22 \%)$ & $482(25 \%)$ & $108(26 \%)$ & $426(24 \%)$ & $112(21 \%)$ & $441(27 \%)$ & $220(26 \%)$ \\
\hline 3 & $495(21 \%)$ & $12(24 \%)$ & $466(22 \%)$ & $46(18 \%)$ & $400(21 \%)$ & $95(23 \%)$ & $387(22 \%)$ & $111(21 \%)$ & $306(19 \%)$ & $173(21 \%)$ \\
\hline 4 & $417(18 \%)$ & $8(16 \%)$ & $372(17 \%)$ & $50(19 \%)$ & $307(16 \%)$ & $66(16 \%)$ & $338(19 \%)$ & $115(22 \%)$ & $278(17 \%)$ & $121(14 \%)$ \\
\hline 5 & $502(22 \%)$ & $8(16 \%)$ & $467(22 \%)$ & $64(25 \%)$ & $298(15 \%)$ & $66(16 \%)$ & $305(17 \%)$ & $121(23 \%)$ & $245(15 \%)$ & $147(17 \%)$ \\
\hline \multicolumn{11}{|l|}{ Charlson score } \\
\hline 0 & $1722(74 \%)$ & $39(80 \%)$ & $1635(76 \%)$ & $200(77 \%)$ & $1607(83 \%)$ & $357(85 \%)$ & $1241(70 \%)$ & $354(66 \%)$ & $1266(77 \%)$ & $635(75 \%)$ \\
\hline 1 & $455(20 \%)$ & $8(16 \%)$ & $41(19 \%)$ & $36(14 \%)$ & $266(14 \%)$ & $51(12 \%)$ & $424(24 \%)$ & $148(28 \%)$ & $304(19 \%)$ & $166(20 \%)$ \\
\hline$\geq 2$ & $147(6 \%)$ & $2(4 \%)$ & $111(5 \%)$ & $23(9 \%)$ & $66(3 \%)$ & $13(3 \%)$ & $111(6 \%)$ & $32(6 \%)$ & $64(4 \%)$ & $43(5 \%)$ \\
\hline \multicolumn{11}{|l|}{ Hypertension } \\
\hline No & $1411(61 \%)$ & $27(55 \%)$ & $1198(56 \%)$ & $137(53 \%)$ & $1262(65 \%)$ & $284(67 \%)$ & $980(55 \%)$ & $286(54 \%)$ & $914(56 \%)$ & $476(56 \%)$ \\
\hline Yes & $913(39 \%)$ & $22(45 \%)$ & $959(45 \%)$ & $122(47 \%)$ & $677(35 \%)$ & $137(33 \%)$ & $796(45 \%)$ & $248(46 \%)$ & $720(44 \%)$ & $368(44 \%)$ \\
\hline \multicolumn{11}{|l|}{ MI } \\
\hline No & $2284(98 \%)$ & $49(100 \%)$ & $2121(98 \%)$ & $255(98 \%)$ & $1932(99 \%)$ & $420(99 \%)$ & $1771(99 \%)$ & $529(99 \%)$ & $1617(99 \%)$ & $837[99 \%]$ \\
\hline Yes & $40(2 \%)$ & $0(0 \%)$ & $36(25)$ & $4(2 \%)$ & $7(0.4 \%)$ & $1(0.2 \%)$ & $5(0.3 \%)$ & $5(1 \%)$ & $17(1 \%)$ & $7(1 \%)$ \\
\hline \multicolumn{11}{|l|}{ DM } \\
\hline No & $1974(85 \%)$ & $44(90 \%)$ & $1836(85 \%)$ & $214(83 \%)$ & $1706(88 \%)$ & $386(92 \%)$ & $1207(68 \%)$ & $348(65 \%)$ & $1298(79 \%)$ & $659(78 \%)$ \\
\hline Yes & $350(15 \%)$ & $5(10 \%)$ & $321(15 \%)$ & $45(17 \%)$ & $233(12 \%)$ & $35(8 \%)$ & $569(32 \%)$ & $186(35 \%)$ & $336(21 \%)$ & $185(22 \%)$ \\
\hline \multicolumn{11}{|l|}{ CKD } \\
\hline No & $2293(99 \%)$ & $48(98 \%)$ & $2093(97 \%)$ & $250(97 \%)$ & $1888(97 \%)$ & $405(96 \%)$ & $1717(97 \%)$ & $510(96 \%)$ & $1424(87 \%)$ & $714(85 \%)$ \\
\hline Yes & $31(1 \%)$ & $1(2 \%)$ & $64(3 \%)$ & $9(3 \%)$ & $51(3 \%)$ & $16(4 \%)$ & $59(3 \%)$ & $24(4 \%)$ & $210(13 \%)$ & $130[15 \%]$ \\
\hline
\end{tabular}

Abbreviations: Abbreviations: RN = radical nephrectomy; PN = partial nephrectomy; NOS = not otherwise specified; ADG = Johns Hopkins' Aggregated Diagnosis Groups; HTN = hypertension; MI = myocardial infarction; $\mathrm{DM}=$ diabetes mellitus. 
Table 2. Multivariable Cox proportional hazards model of risk of requiring renal replacement therapy with Charlson comorbidity score

\begin{tabular}{|l|l|l|}
\hline & HR (CI) & p-value \\
\hline Surgery & & \\
\hline RN & Ref & \\
\hline PN & $1.31(0.94-1.82)$ & 0.10 \\
\hline Sex & & \\
\hline Female & Ref & \\
\hline Male & $1.14(0.89-1.45)$ & 0.28 \\
\hline Age group & & \\
\hline$<50$ & Ref & \\
\hline $50-59$ & $1.24(0.80-1.93)$ & 0.32 \\
\hline $60-69$ & $1.72(1.14-2.59)$ & $<0.05$ \\
\hline $70-79$ & $2.11(1.40-3.18)$ & $<0.05$ \\
\hline$\geq 80$ & $1.53(0.82-2.86)$ & 0.17 \\
\hline Year group & & \\
\hline $1995-1999$ & Ref & \\
\hline $2000-2004$ & $0.97(0.73-1.28)$ & 0.83 \\
\hline $2005-2010$ & $0.56(0.39-0.80)$ & $<0.05$ \\
\hline Histology & & \\
\hline Clear cell & Ref & \\
\hline Papillary & $1.20(0.63-2.31)$ & 0.57 \\
\hline Chromophobe & $0.79(0.28-2.19)$ & 0.65 \\
\hline NOS & $1.59(1.16-2.19)$ & $<0.05$ \\
\hline Charlson & & \\
\hline 0 & Ref & \\
\hline 1 & $3.27(2.51-4.26)$ & $<0.05$ \\
\hline$\geq 2$ & $7.48(5.46-10.26)$ & $<0.05$ \\
\hline & & \\
\hline & & \\
\hline 22 & & \\
\hline & & \\
\hline & & \\
\hline
\end{tabular}

Abbreviations: $\mathrm{HR}=$ hazards ratio; $\mathrm{CI}$ = confidence interval; $\mathrm{RN}=$ radical nephrectomy; $\mathrm{PN}$ = partial nephrectomy; NOS = not otherwise specified; 
Table 3. Multivariable Cox proportional hazards model of risk of requiring renal replacement therapy with weighted ADG score

\begin{tabular}{|l|l|l|}
\hline & HR (CI) & p-value \\
\hline Surgery & & \\
\hline RN & Ref & \\
\hline PN & $1.31(0.95-1.83)$ & 0.10 \\
\hline Sex & & \\
\hline Female & Ref & \\
\hline Male & $1.22(0.96-1.56)$ & 0.09 \\
\hline Age group & & \\
\hline$<50$ & Ref & \\
\hline $50-59$ & $1.27(0.82-1.97)$ & 0.27 \\
\hline $60-69$ & $1.67(1.11-2.52)$ & $<0.05$ \\
\hline $70-79$ & $2.05(1.35-3.09)$ & $<0.05$ \\
\hline$\geq 80$ & $1.41(0.75-2.65)$ & 0.27 \\
\hline Year group & & \\
\hline $1995-1999$ & Ref & \\
\hline $2000-2004$ & $0.99(0.75-1.32)$ & 0.98 \\
\hline $2005-2010$ & $0.58(0.40-0.83)$ & $<0.05$ \\
\hline Histology & & \\
\hline Clear cell & Ref & \\
\hline Papillary & $1.05(0.55-2.02)$ & 0.87 \\
\hline Chromophobe & $0.75(0.27-2.08)$ & 0.58 \\
\hline NOS & $1.64(1.19-1.07)$ & $<0.05$ \\
\hline ADG (weighted) & & \\
\hline & $1.06(1.04-1.07)$ & $<0.05$ \\
\hline & & \\
\hline & & \\
\hline
\end{tabular}

Abbreviations: $\mathrm{HR}=$ hazards ratio; $\mathrm{CI}=$ confidence interval; $\mathrm{RN}=$ radical nephrectomy; $\mathrm{PN}=$ partial nephrectomy; NOS = not otherwise specified; ADG = Johns Hopkins' Aggregated Diagnosis Groups. 
Table 4. Cox proportional hazards model adjusting for propensity score as a continuous and categorical variable for the outcome of a) ESRD b) ESRD (modern cohort) c) new onset CKD d) myocardial infarction e) acute dialysis f) overall survival g) cancer-specific survival

a)

\begin{tabular}{|c|c|c|c|c|}
\hline & \multicolumn{4}{|c|}{ ESRD } \\
\hline & \multicolumn{2}{|l|}{ Continuous PS } & \multicolumn{2}{|l|}{ Categorical PS } \\
\hline & $\mathrm{HR}(\mathrm{CI})$ & p-value & $\mathrm{HR}(\mathrm{CI})$ & $\mathrm{p}$-value \\
\hline Surgery & & & & \\
\hline $\mathrm{RN}$ & Ref & & Ref & \\
\hline $\mathrm{PN}$ & $1.04(0.75-1.45)$ & 0.79 & $1.07(0.77-1.49)$ & 0.66 \\
\hline $\begin{array}{l}\text { Propensity score } \\
\text { (continuous) }\end{array}$ & $1.55(0.47-5.15)$ & 0.47 & $\mathrm{n} / \mathrm{a}$ & \\
\hline $\begin{array}{l}\text { Propensity score } \\
\text { (quintiles) }\end{array}$ & & & & \\
\hline Q1 & $\mathrm{n} / \mathrm{a}$ & & Ref & \\
\hline Q2 & $\mathrm{n} / \mathrm{a}$ & & $1.18(0.86-1.61)$ & 0.30 \\
\hline Q3 & $\mathrm{n} / \mathrm{a}$ & & $0.71(0.48-1.04)$ & 0.08 \\
\hline Q4 & $\mathrm{n} / \mathrm{a}$ & & $0.79(0.52-1.21)$ & 0.29 \\
\hline Q5 & $\mathrm{n} / \mathrm{a}$ & & $1.26(0.86-1.85)$ & 0.22 \\
\hline
\end{tabular}

Abbreviations: $\mathrm{ESRD}=$ end stage renal disease; $\mathrm{PS}=$ propensity score; $\mathrm{HR}=$ hazards ratio; $\mathrm{CI}=$ confidence interval; $\mathrm{RN}=$ radical nephrectomy; $\mathrm{PN}$ = partial nephrectomy.

b)

\begin{tabular}{|c|c|c|c|c|}
\hline & \multicolumn{4}{|c|}{ ESRD (modern cohort) } \\
\hline & \multicolumn{2}{|l|}{ Continuous PS } & \multicolumn{2}{|l|}{ Categorical PS } \\
\hline & $\mathrm{HR}(\mathrm{CI})$ & p-value & $\mathrm{HR}(\mathrm{CI})$ & p-value \\
\hline \multicolumn{5}{|l|}{ Surgery } \\
\hline $\mathrm{RN}$ & Ref & & Ref & \\
\hline PN & $0.48(0.28-0.82)$ & $<0.05$ & $0.55(0.30-0.89)$ & $<0.05$ \\
\hline $\begin{array}{l}\text { Propensity score } \\
\text { (continuous) }\end{array}$ & $644(73-5672)$ & $<0.05$ & $\mathrm{n} / \mathrm{a}$ & \\
\hline \multicolumn{5}{|l|}{$\begin{array}{l}\text { Propensity score } \\
\text { (quintiles) }\end{array}$} \\
\hline Q1 & $\mathrm{n} / \mathrm{a}$ & & Ref & \\
\hline $\mathrm{Q} 2$ & $\mathrm{n} / \mathrm{a}$ & & 149 (n/a) & $<0.05$ \\
\hline Q3 & $\mathrm{n} / \mathrm{a}$ & & $144(\mathrm{n} / \mathrm{a})$ & $<0.05$ \\
\hline Q4 & $\mathrm{n} / \mathrm{a}$ & & $117(\mathrm{n} / \mathrm{a})$ & $<0.05$ \\
\hline Q5 & $\mathrm{n} / \mathrm{a}$ & & $315(n / a)$ & $<0.05$ \\
\hline
\end{tabular}


c)

\begin{tabular}{|l|l|l|l|l|}
\hline & \multicolumn{4}{|l|}{ New onset CKD } \\
\hline & \multicolumn{2}{|l|}{ Continuous PS } & Categorical PS & p-value \\
\hline & HR (CI) & p-value & HR (CI) & \\
\hline Surgery & & & & \\
\hline RN & Ref & $<0.05$ & $0.52(0.44-0.61)$ & $<0.05$ \\
\hline PN & $0.50(0.43-0.59)$ & n/a & \\
\hline $\begin{array}{l}\text { Propensity score } \\
\text { (continuous) }\end{array}$ & $43.65(26.81-71.05)$ & $<0.05$ & & \\
\hline $\begin{array}{l}\text { Propensity score } \\
\text { (quintiles) }\end{array}$ & & & & \\
\hline Q1 & n/a & & Ref & \\
\hline Q2 & n/a & & $1.96(1.73-2.22)$ & $<0.05$ \\
\hline Q3 & n/a & & $1.89(1.63-2.19)$ & $<0.05$ \\
\hline Q4 & n/a & & $3.82(3.27-4.48)$ & $<0.05$ \\
\hline Q5 & n/a & $2.96(2.48-3.53)$ & $<0.05$ \\
\hline
\end{tabular}

d)

\begin{tabular}{|l|l|l|l|l|}
\hline & \multicolumn{4}{|l|}{ Myocardial infarction } \\
\hline & \multicolumn{2}{|l}{ Continuous PS } & Categorical PS & p-value \\
\hline & HR (CI) & p-value & HR (CI) & \\
\hline Surgery & & & & \\
\hline RN & Ref & & Ref & \\
\hline PN & $0.81(0.55-1.19)$ & 0.81 & $0.81(0.55-1.20)$ & 0.30 \\
\hline $\begin{array}{l}\text { Propensity score } \\
\text { continuous) }\end{array}$ & $0.03(0.007-0.10)$ & $<0.05$ & n/a & \\
\hline $\begin{array}{l}\text { Propensity score } \\
\text { (quintiles) }\end{array}$ & & & & \\
\hline Q1 & n/a & & Ref & \\
\hline Q2 & n/a & & $0.79(0.60-1.03)$ & 0.08 \\
\hline Q3 & n/a & & $0.49(0.35-0.68)$ & $<0.05$ \\
\hline Q4 & n/a & & $0.46(0.30-0.70)$ & $<0.05$ \\
\hline Q5 & n/a & & $0.33(0.20-0.54)$ & $<0.05$ \\
\hline
\end{tabular}


e)

\begin{tabular}{|l|l|l|l|l|}
\hline & \multicolumn{4}{|l|}{ Acute dialysis } \\
\hline & \multicolumn{2}{|l|}{ Continuous PS } & Categorical PS & p-value \\
\hline & HR (CI) & p-value & HR (CI) & \\
\hline Surgery & & & Ref & \\
\hline RN & Ref & 0.42 & $1.16(0.85-1.58)$ & 0.34 \\
\hline PN & $1.13(0.83-1.55)$ & 0.84 & n/a & \\
\hline $\begin{array}{l}\text { Propensity score } \\
\text { (continuous) }\end{array}$ & $1.12(0.35-3.57)$ & & & \\
\hline $\begin{array}{l}\text { Propensity score } \\
\text { (quintiles) }\end{array}$ & & & Ref & \\
\hline Q1 & n/a & & $1.08(0.79-1.47)$ & 0.61 \\
\hline Q2 & n/a & & $0.74(0.51-1.06)$ & 0.10 \\
\hline Q3 & n/a & & $0.73(0.48-1.09)$ & 0.12 \\
\hline Q4 & n/a & & $1.13(0.78-1.64)$ & 0.50 \\
\hline Q5 & n/a & &
\end{tabular}

f)

\begin{tabular}{|l|l|l|l|l|}
\hline & \multicolumn{4}{|l|}{ Overall survival } \\
\hline & \multicolumn{2}{|l|}{ Continuous PS } & Categorical PS & p-value \\
\hline & HR (CI) & p-value & HR (CI) & \\
\hline Surgery & & & Ref & \\
\hline RN & Ref & $<0.05$ & $0.44(0.37-0.51)$ & $<0.05$ \\
\hline PN & $0.45(0.38-0.52)$ & $<0.05$ & n/a & \\
\hline $\begin{array}{l}\text { Propensity score } \\
\text { (continuous) }\end{array}$ & $0.07(0.05-0.11)$ & & & \\
\hline $\begin{array}{l}\text { Propensity score } \\
\text { (quintiles) }\end{array}$ & & & Ref & \\
\hline Q1 & n/a & & $0.93(0.85-1.02)$ & 0.16 \\
\hline Q2 & n/a & & $0.56(0.50-0.63)$ & $<0.05$ \\
\hline Q3 & n/a & & $0.62(0.55-0.71)$ & $<0.05$ \\
\hline Q4 & n/a & & $0.49(0.42-0.58)$ & $<0.05$ \\
\hline Q5 & n/a & &
\end{tabular}

g)

\begin{tabular}{|l|l|l|l|l|}
\hline & \multicolumn{4}{|c|}{ Cancer-specific survival } \\
\hline & Continuous PS & \multicolumn{2}{l|}{ Categorical PS } \\
\hline & HR (CI) & p-value & HR (CI) & p-value \\
\hline Surgery & & & Ref & \\
\hline RN & Ref & $<0.05$ & $0.21(0.14-0.30)$ & $<0.05$ \\
\hline PN & $0.21(0.15-0.31)$ & n/a & \\
\hline $\begin{array}{l}\text { Propensity score } \\
\text { (continuous) }\end{array}$ & $0.007(0.003-0.01)$ & $<0.05$ & & \\
\hline $\begin{array}{l}\text { Propensity score } \\
\text { (quintiles) }\end{array}$ & & & & \\
\hline Q1 & n/a & & Ref & \\
\hline Q2 & n/a & & $0.79(0.69-0.91)$ & $<0.05$ \\
\hline Q3 & n/a & & $0.51(0.43-0.61)$ & $<0.05$ \\
\hline Q4 & n/a & & $0.32(0.25-0.40)$ & $<0.05$ \\
\hline Q5 & n/a & $0.20(0.14-0.27)$ & $<0.05$ \\
\hline
\end{tabular}


Table 5. Baseline characteristics of the modern cohort (2003-2010)

\begin{tabular}{|c|c|c|c|}
\hline & Radical nephrectomy & Partial nephrectomy & Overall \\
\hline Patients & $5,484(76 \%)$ & $1,746(24 \%)$ & 7,230 \\
\hline Median follow-up (mo) & $46(24-72)$ & $36(24-60)$ & $41(24-65)$ \\
\hline \multicolumn{4}{|l|}{ Sex } \\
\hline Male & $3,328(61 \%)$ & $1,099(63 \%)$ & $4,427(61 \%)$ \\
\hline Female & $2,156(39 \%)$ & $647(37 \%)$ & $2,803(39 \%)$ \\
\hline \multicolumn{4}{|l|}{ Age group } \\
\hline$<50$ & $952(17 \%)$ & $436(25 \%)$ & $1,388(19 \%)$ \\
\hline $50-59$ & $1,400(26 \%)$ & $496(28 \%)$ & $1,896(26 \%)$ \\
\hline $60-69$ & $1,534(28 \%)$ & $451(26 \%)$ & $1,985(27 \%)$ \\
\hline $70-79$ & $1,194(22 \%)$ & $313(18 \%)$ & $1,507(21 \%)$ \\
\hline$\geq 80$ & $404(7 \%)$ & $50(3 \%)$ & $454(6 \%)$ \\
\hline \multicolumn{4}{|l|}{ Histology } \\
\hline Clear cell & $2,307(42 \%)$ & $674(39 \%)$ & $2,981(41 \%)$ \\
\hline Papillary & $450(8 \%)$ & $198(11 \%)$ & $648(9 \%)$ \\
\hline Chromophobe & $332(6 \%)$ & $123(7 \%)$ & $455(6 \%)$ \\
\hline NOS & $2,395(44 \%)$ & $751(43 \%)$ & $3,146(44 \%)$ \\
\hline \multicolumn{4}{|l|}{ Weighted ADG (quintile) } \\
\hline 1 (least comorbidity) & $1,044(19 \%)$ & $325(19 \%)$ & $1,369(19 \%)$ \\
\hline 2 & $1,329(24 \%)$ & $419(24 \%)$ & $1,748(24 \%)$ \\
\hline 3 & $1,127(21 \%)$ & $378(22 \%)$ & $1,505(21 \%)$ \\
\hline 4 & $994(18 \%)$ & $304(17 \%)$ & $1,298(18 \%)$ \\
\hline 5 & $990(18 \%)$ & $320(18 \%)$ & $1,310(18 \%)$ \\
\hline \multicolumn{4}{|l|}{ Charlson score } \\
\hline 0 (least comorbidity) & $4,201(77 \%)$ & $1,320(76 \%)$ & $5,521(76 \%)$ \\
\hline 1 & $1,031(19 \%)$ & $342(20 \%)$ & $1,373(19 \%)$ \\
\hline$\geq 2$ & $252(5 \%)$ & $84(5 \%)$ & $366(5 \%)$ \\
\hline \multicolumn{4}{|l|}{ Hypertension } \\
\hline No & $3,073(56 \%)$ & $1,015(58 \%)$ & $4,088(56 \%)$ \\
\hline Yes & $2,411(44 \%)$ & $731(42 \%)$ & $3,142(43 \%)$ \\
\hline \multicolumn{4}{|l|}{ Diabetes } \\
\hline No & $4,350(79 \%)$ & $1,354(78 \%)$ & $5,704(79 \%)$ \\
\hline Yes & $1,134(21 \%)$ & $392(22 \%)$ & $1,526(21 \%)$ \\
\hline \multicolumn{4}{|l|}{ CKD } \\
\hline No & $5,179(94 \%)$ & $1,601(92 \%)$ & $6,780(94 \%)$ \\
\hline Yes & $305(6 \%)$ & $145(8 \%)$ & $450(6 \%)$ \\
\hline \multicolumn{4}{|l|}{ Myocardial infarction } \\
\hline No & $5,428(99 \%)$ & $1,737(99.5 \%)$ & $7,165(99 \%)$ \\
\hline Yes & $56(1 \%)$ & $9(0.5 \%)$ & $65(1 \%)$ \\
\hline
\end{tabular}

Abbreviations: $\mathrm{mo}=$ months; $\mathrm{NOS}=$ not otherwise specified; $\mathrm{ADG}=$ Johns Hopkins' Aggregated Diagnosis Groups; $\mathrm{CKD}=$ chronic kidney disease 


\section{APPENDIX B}

TORONTIO

PROTOCOL REFERENCE \# 27211

January 23, 2013

Dr. Shabbir Alibhai
DEPT OF MEDICINE
FACULTY OF MEDICINE
Office of the Vice President, Research

Dear Dr. Alibhai and Dr. Stanley Yap,

Re: Your research protocol entitled, "Partial nephrectomy for the treatment of renal cell carcinoma and the risk of end stage renal disease" ETHICS APPROVAL

Dr. Stanley Yap

DEPT OF MEDICINE

FACULTY OF MEDICINE

Expiry Date: January 18, 2014

Continuing Review Level: 1

Renewal: Data Analysis Only

We are writing to advise you that you have been granted annual renewal of ethics approval to the above-referenced research protocol through the Research Ethics Board (REB) delegated process.

Please note that all protocols involving ongoing data collection or interaction with human participants are subject to re-evaluation after 5 years. Ongoing research under this protocol must be renewed prior to the expiry date.

Please ensure that you submit an Annual Renewal Form or a Study Completion Report 15 to 30 days prior to the expiry date of your protocol. Note that annual renewals for protocols cannot be accepted more than 30 days prior to the date of expiry as per our guidelines.

Any changes to the approved protocol or consent materials must be reviewed and approved through the amendment process prior to its implementation. Any adverse or unanticipated events should be reported to the Office of Research Ethics as soon as possible. If your research is funded by a third party, please contact the assigned Research Funding Officer in Research Services to ensure that your funds are released.

Best wishes for the successful completion of your research.

Yours sincerely,

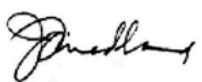

Judith Friedland, Ph.D REB Chair

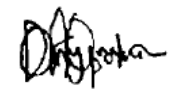

Daniel Gyewu REB Manager 